\title{
PROCEEDINGS OF THE FORTY-FIRST ANNUAL MEETING OF THE AMERICAN SOCIETY FOR CLINICAL INVESTIGATION HELD IN ATLANTIC CITY, N. J., MAY 2, 1949
}

\author{
READ BEFORE THE SCIENTIFIC SESSION
}

Presidential Address

\section{CLINICAL RESEARCH AS A CAREER}

\author{
BY WESLEY W. SPINK
}

On May 10, 1909, Dr. S. J. Meltzer, the first President of this Society, addressed the members on the subject, "The Science of Clinical Medicine: What it Ought to be and the Men to Uphold it." In his introductory remarks he said, "The honor of the presidency of a society goes with the privilege of discussing some of the aims of that society." In discharging my responsibility as President, forty years after Dr. Meltzer, I am tempted to review the accomplishments of this Society, but such would be idle boasting. I would like to call your attention to the history of this Society by Dr. J. Harold Austin of Philadelphia which appears in the March, 1949 issue of the Journal of Clinical Investigation. It is well worth reading. Dr. Meltzer was deeply interested in the capable young individual who elected a career of clinical research. Since today, more than ever, young individuals are continuing to manifest an interest in clinical investigation, I would like to discuss with you some of the problems relating to their training.

\section{The Clinical Investigator as a Clinician}

Clinical investigation implies the critical study of human beings in sickness and in health. This suggests many ramifications of effort. While fruitful studies on human disease may be pursued within the confines of laboratory walls without the investigator ever coming in contact with patients, someone has had to define the problem or problems in disease that the patients present. It cannot be emphasized too strongly in this day of highly specialized techniques and laboratory research that successful clinical investigation is dependent upon a knowledge of disease in human beings. If one is setting out to resolve a problem in human disease, it is desirable to know as much as possible about the natural history of that disease. This information is not to be gleaned in an armchair, pouring over books and journals, but rather by the persistent observation of patients on the wards and in the dispensaries. The individual interested in a career of investigation should pursue further clinical training after completing his internship by selecting an appointment in which he will be given responsibility for the care of patients and in an institution where he will have the time and facilities to engage in research. Competent advisors should be readily available to aid him in his clinical activities and to lend direction to his investigations.

What attitude should the resident in clinical medicine interested in a career of research and academic medicine take toward certification by one of the specialty boards of medicine? This is not the place to discuss the trend toward specialization in medicine as reflected by the specialty boards. But I see no valid reason why the resident should not fulfill the requirements of certification while he is obtaining his training. If he expects some day to have an academic appointment of any merit, in which he will be responsible for the clinical training of undergraduate and graduate medical students, he should at least possess that general knowledge of medicine which is necessary to pass the examinations of a specialty board. Furthermore, after he has had his formal residency training and has had an opportunity to engage in research activities, he may desire to go into the practice of medicine, or, much against his wishes, economic circumstances and obligations may make it mandatory that he enter into care of his own patients. Engaged in the private practice of medicine as a highly trained clinician, certification by a specialty board will be a decided advantage. However, it is diffcult to lend a sympathetic ear to the individual whose primary interest in a residency is to fulfill the requirements of a specialty board and who applies for an appointment on a service devoted to teaching and clinical investigation with this purpose in mind.

\section{The Clinical Investigator and the Basic Sciences}

Clinical investigation today, contending as it does with the complexities of human disease and the assimilation of quantitative information, has narrowed the possibilities of carrying out fundamental research at the bedside of the patient. Highly technical knowledge is essential and the special tools of a basic discipline are frequently needed. The clinician may obtain help with his problem by turning to his colleagues in the basic sciences or to skilled laboratory technologists. Under these circumstances, he throws himself on the mercy of others for the accuracy of the acquired data and for their interpretation. It is difficult to evaluate laboratory results if one is unacquainted with the basic principles involved, and one cannot seek out the sources of error if he is ignorant of laboratory methods. Therefore, in the prosecution of clinical research, it is frequently necessary for the clinician to take time out and go into the laboratory. A year or two may be devoted to one of the basic sciences such as physiology, bacteriology, or pharmacology. Today it is not unusual for clinicians to concentrate on a review of the advances in the fields of chemistry or physics. A brilliant chapter in the history of clinical investigation includes modern cardiovascular surgery 
achieved by the amalgamation of basic physiologic principles and improved surgical techniques worked out in the laboratory on lower animals. The surgical correction of coarctation of the aorta was not evolved by the method of trial and error on human subjects, but the techniques emanated from the laboratory after months of painstaking thought and effort.

\section{The Clinical Investigator and the Research Team}

One of the developments in recent years in clinical research has been the organization of personnel as a team in the prosecution of a problem. This tendency toward joint effort was accelerated during the last war. For many projects there are distinct advantages that accrue from pooling the abilities of several investigators. Among the disadvantages lurking in such an endeavor is the shackling of the independence of thought and initiative of young investigators. Any individual who wants to do original work in clinical research must be extremely discriminating in electing to participate in a large project lest his freedom of thought and action be smothered by the desires of his supervisors. This trend toward team research applies not only to the medical sciences but also to other fields.

\section{The Clinical Investigator and the Preparation of Data for Publication}

It is unfortunate that many investigators in the course of their training have not been given direction in communicating their results to others. While this applies also to the verbal presentation of data, I am particularly concerned with the preparation of manuscripts for publication. Such an endeavor often represents several years of work by the individual, and an institution may have expended thousands of dollars in giving that person an opportunity to carry out the research. And yet it is pathetic to see the results embodied in an inarticulate exposition. That young investigators are not being given guidance is manifested by the appeal made by the editors of the Journal of Clinical Investigation in the issue for September, 1948, when they cited the deficiencies of papers being submitted to them and asked that more diligence and care be given to the preparation of a manuscript. Some principal investigators are so engrossed with their own research and personal interests that they have neither the time nor the concern for aiding the young investigator in the assimilation of his data and preparing them for publication. The writing of a scientific paper is not easy, and there are no short cuts in preparing an accurate and readable report.

\section{The Clinical Investigator and His Economic Status}

Individuals who elect to spend three or more years as a resident in one of the clinical disciplines face the grim reality that the financial return at best is but sufficient to meet the basic needs of existence. It is to be regretted that this applies to those who are encouraged to extend this postgraduate period of training and continue on with clinical investigation. Considerable admiration must be extended to the courage of the war veteran with a wife and children who has made the decision to stay with clinical research. Too often, able individuals find it utterly impossible to continue on with the stipends accorded them, and they drop out and go into more lucrative fields of medical endeavor, especially private practice. It has become quite obvious that medical schools, hospitals, and institutes of research cannot satisfactorily finance the medical program of graduate training in medicine. It is also apparent that private enterprise in general cannot continue to support medical research as in the past. Where, then, are the institutions charged with the responsibility of training clinical investigators to turn for financial aid? Major support today is coming from the Federal Government. The taxpayer is footing large expenditures of funds for medical education and research. Because of this postwar development, I would like to discuss briefly Federal aid that is coming from three sources. First, a most remarkable union has been made between medical education and the Veterans Administration. This joint enterprise has resulted in an excellent training program for residents in the various specialties, and it has encouraged sound clinical investigation. The end result has not only been of considerable aid to the medical schools in discharging their obligations for postgraduate medical training to the veterans, but the patients have benefited from the skilled medical care. If a large segment of clinical material is to be hospitalized under the Veterans Administration in the future as at the present time, it is absolutely essential that medical schools continue with this cooperative effort. But the union will only continue successfully if the same high standards set up by the Deans' Committees and the Veterans Administration continue in force. A second type of Federal aid to medical education and research is that manifested by the United States Public Health Service. These funds have been administered wisely and have supported many excellent research projects. In addition, the Public Health Service has fostered a modest but sound program of education and research for promising young individuals. A third type of Federal aid to education and research is that distributed by the military branches. It seems almost incredible that in a free society desperately seeking ways to achieve world peace, tremendous sums of money should be allocated to military agencies for applied and fundamental research, particularly when much of the research has no direct bearing on military activities. While freedom of thought and action in research has accompanied the Army and Navy grants for medical investigations, it should never be forgotten that anything earmarked military implies security and that, under these circumstances, freedom may be stifled overnight in the interests of "national security."

Some young investigators are sorely grieved, and perhaps rightly so, at the scanty financial returns afforded them during their formative years. If these individuals anticipate later on in life an income commensurate with their labor and contributions to medical research, they will be sadly disappointed. As the young investigator matures, and after he has established his ability as a 
clinician and as a scientist, he is often faced with the serious decision as to whether he can continue in the field of medical research on a full-time basis. Too many times, economic circumstances demand that he compromise and attempt the blending of the private practice of medicine with clinical research. It is not my purpose to belabor the question of private practice versus the fulltime clinical investigator, but this problem confronts most men who are attempting to develop and direct medical research. As he concluded his address to the first meeting of this Society, Dr. Meltzer stated, "Teaching medicine and furthering its science is a very serious business which ought to be carried on by men who are ready to devote all or most of their time to it-but let me tell you : beware of practice. It is a bewitching graveyard in which many a brain has been buried alive with no other compensation than a gilded tombstone." Twenty years later, another great figure in clinical research, Sir Thomas Lewis, wrote, "No investigator can be successful who allows, or is forced by circumstances to allow, solicitude for his patients to preoccupy his mind." While it would be highly desirable today for competent young men to continue on a full-time basis, we cannot escape the bald fact that hospitals and medical schools are able to support in this manner but a very limited number of these individuals. I am of the opinion that a man can carry on a restricted private practice and engage in clinical research. Under these conditions, a clinician is often a better teacher and, if clinical investigation means the study of human disease, patients coming to him for advice may be of considerable aid in the resolving of a clinical problem. But unfortunately, the well-trained clinician possessed of desirable personal characteristics is often sought after by other physicians and patients to the detriment of his research. A soaring income accompanied by an elevated social position frequently has more enchantment than the self-sacrifice and many frustrations that go with clinical research.

The Clinical Investigator and His Obligation to Society

The era in which we are living has been called the Scientific Age, and now we are told about the coming Atomic Age. Society with its "cold wars" and anxious tensions expects and needs leadership from scientists. While the attention of the public is centered chiefly on those working in the fields of nuclear physics and electronics, the medical scientist has also grown in stature. Is the medical man prepared to assume his responsibility of leadership? One of the undesirable aspects of medical education is the emphasis placed upon the natural sciences and the scant attention given to the study of history, political economy, and the social sciences. When the student of medicine in this country finishes his undergraduate and postgraduate education, he has received the finest technologic training available in the world today. But he has not been educated for a position of leadership in society at large. He does not know, nor often does he appear to care, about the social, political, and economic changes disturbing the equilibrium of society. In his zeal to unravel a mystery of pathologic physiolgy at the bedside or in the laboratory, he has no time for the problems of the world with its clashing ideologies, opposing cultures, social hatreds, and nationalistic pride masking economic greed. The medical school curriculum could stand a little more emphasis upon social pathology, even at the expense of morbid anatomy. The young clinical investigator is the leader of medical education tomorrow. In concluding my remarks this morning, I would like to plead that the young medical scientists do not hide within their ivory towers of learning, oblivious of the community and its problems. As the investigator pursues his training, let him give time and thought to the immediate issues facing mankind. As he attempts to mold and influence the thinking and attitudes of medical students under him, let him remind his charges that the possessor of a medical degree is a humanitarian rather than merely a doctor dispensing his technologic skill to the highest bidder. Let him instill within his students the desire to acquire a social conscience. Let the young instructor inculcate upon his classes the fact that environmental factors are often more important in the genesis of human illness than organic changes. Finally, he knows and his students know that the distribution of medical care will undergo some changes. Let him beseech his students as they attempt to resolve this problem in their own minds that they utilize the experimental method in which conclusions are reached only after accurate data have been assimilated rather than on the basis of ignorance and selfishness. 


\section{ABSTRACTS}

Value of Blood and Urine Lactate Response to Physical Effort as an Index of Physical Fitness. DAvid I. Abramson, and (by invitation) Robert W. KeEton, Warren H. Cole, H. H. Mitchell, J. M. Dyniewicz, M. C. Gephardt, S. J. Presley, and W. R. Kearney, Chicago, Ill.

The effects of various work loads on the lactic acid in the blood and urine were studied in a group of patients in widely different states of physical fitness as judged by clinical standards. When all were subjected to the same small amount of work on a treadmill, the individuals in good physical condition showed much less of an increase in the blood lactate level, with the peak of the curve appearing sooner, than was observed in those in poor shape. The patients falling in categories between the two extremes demonstrated changes which could be correlated with their relative states of physical fitness. From the alterations noted in urine lactate content, the patients could only grossly be divided into good or poor performers.

When the individuals walked on the treadmill almost to the point of exhaustion, the variations in blood lactate response were such that further subdivisions with regard to gradations in physical fitness were made possible. In the patient in excellent shape, the blood lactate curve showed a peak, occurring at a relatively low point, early in the exercise and then a fall during the remainder of the walk; this continued into the post-exercise period. The individual in the next lower category did not demonstrate the fall during the latter part of exercise but a plateau instead. The patient in poor physical shape showed an ascending slope during exercise and a peak, occurring at a much higher level, late in the post-exercise period.

Following a major surgical procedure and its associated period of physical inactivity, many of the patients demonstrated alterations in the blood lactate curve which were interpreted as a decrement in the efficiency of their reaction to the work load.

It is concluded that the blood lactate response to exercise is a sensitive index of physical fitness.

Genetic Studies of 201 Persons with Hereditary Hypercholesterolemia (Thirty-five Families and Twenty-nine Individuals). David Adlersberg and AlberT D. Parets (Introduced by Ernest P. Boas), New York, N. Y.

Continuing previous studies, 35 families (172 members) and 29 individual patients with hereditary hypercholesterolemia (Familial Xanthomatosis), totalling 201 persons, were investigated. Forty-nine patients ( 24 per cent) exhibited the full syndrome of hypercholesterolemia, coronary artery disease and one or more of the following : xanthoma tuberosum or tendinosum, xanthelasma and juvenile or senile corneal arcus; 122 had hypercholestrolemia (300 mg. per cent or more) ; 61 exhibited xanthelasma; 25 ex- hibited xanthoma and 35, corneal arcus. Coronary artery disease was present in 80 patients (40 per cent). Among these, serum cholesterol was determined in 66 and was found elevated above $300 \mathrm{mg}$. per cent in 57 . Thus, the two most frequent abnormalities encountered in these xanthoma families were hypercholesterolemia and coronary atherosclerosis. Xanthelasma and arcus were next in frequency, while xanthoma was found least often. Genetic analysis revealed that the inborn error of cholesterol metabolism was inherited as an incomplete dominant trait. The significance of these findings in the etiology of coronary artery disease is discussed.

The Role of Phospholipids in High-Lipid Sera in Cirrhosis and Nephrosis. Edward H. Ahrens, JR., and HenRy G. KUNKEL (Introduced by Sidney Rothbard), New York, N. Y.

In a large series of lipemic (milky) sera from $15 \mathrm{pa}$ tients with nephrosis and non-lipemic high-lipid sera from 20 patients with biliary cirrhosis the ratio of phospholipids to total lipids (PL/TL) was found to be high in non-lipemic sera and low in lipemic sera. In fasting sera with total lipids ranging from 600 to $2,900 \mathrm{mg}$. per cent, PL/TL in non-lipemic sera was 0.29 or higher in 22 of 24 instances, whereas in lipemic sera PL/TL was 0.25 or lower in 15 of 19 instances. These data suggest that the clarity of high-lipid sera in biliary cirrhosis is explained by the characteristically large proportion of phospholipids, since these lipids are markedly hydrophilic by nature of their water-soluble side-chains. By this concept lipemia is due to a low proportion of hydrophilic lipids rather than, as commonly postulated, to an absolute increase of neutral fat.

This hypothesis was tested by destroying lecithin and sphingomyelin of serum enzymatically, using $\mathrm{Cl}$. welchii lecithinase which splits phosphorylcholine from lecithin and sphingomyelin, depriving these lipids of their hydrophilic side-chains and leaving water-soluble diglycerides. Exposure of normal and high-lipid sera to this enzyme produced a lipemia indistinguishable grossly and microscopically from that of nephrosis, and the degree of turbidity measured spectrophotometrically was linearly proportional to the total lipid content.

Further studies showed that under certain conditions measurable enzymatic hydrolysis of serum phospholipids occurred before turbidity appeared or increased. Thus, it was demonstrated in high-lipid sera of nephrosis as well as cirrhosis that phospholipids exert an appreciable solubilizing effect upon grossly elevated hydrophobic lipids.

These data have possible application in studies of fat transport and deposition of fat in tissues.

\section{A New Theory of Blood Coagulation with Particular Ref-} erence to the Role of Prothrombin Conversion Accelera- 
tors. Benjamin Alexander and (by invitation), Greta Landwehr and Robert Goldstein, Boston, Mass.

Serum contains a substance which, arising during coagulation, can accelerate the conversion of prothrombin in plasma to thrombin by thromboplastin plus calcium. Many of its properties have been delineated, and it has been separated in a fraction comprising $20 \mathrm{mgm}$. of protein from $100 \mathrm{cc}$. of serum. Evidence indicates that it is a new clotting factor, distinct from Ac-globulin of Ware and Seegers, Factors V and VI of Owren, or Labile Factor of Quick.

The amount of accelerator which evolves during coagulation is related to the amount of prothrombin consumed. In hypoprothrombinemia-congenital, dicumarol induced, or resulting from liver disease-abnormally small amounts of accelerator are elaborated. Furthermore, the interaction of purified prothrombin preparations with thromboplastin and calcium yields substantial amounts of accelerator in addition to thrombin. Accordingly, it is proposed that prothrombin (M.W. 140,000) may be split during its conversion to give thrombin (M.W. 70,000) and the accelerator. This concept is suggested also by remarkable similarity in some respects between prothrombin and the accelerator.

The agent cannot accelerate prothrombin conversion in the absence of a non-prothrombin factor present in fresh plasma. Also, a short interval (approximately 2 minutes) is required for these components to interact. The plasma moiety is labile and appears identical with Labile Factor, Factor V and Plasma Ac-globulin, all of which, it is generally agreed, are probably the same.

On the basis of the above a new theory of blood coagulation is proposed: in the presence of thromboplastin and ionized calcium the prothrombin and serum is slowly cleaved to yield thrombin and serum accelerator. Under the influence of the serum accelerator plasma Ac-globulin (Factor V, Labile Factor) is converted to serum Acglobulin which then accelerates the conversion of additional prothrombin to thrombin and more serum accelerator.

The overall reaction thus proceeds with ever increasing velocity until the prothrombin is almost entirely consumed. Reduction in serum accelerator after dicumarol administration rather than the hypoprothrombinemia per se may explain the efficacy of this drug in the prevention and treatment of thromboembolic disease.

Renal Function in Multiple Myeloma. JoHN B. ARMsTrong (Introduced by Jerome Harris), Durham, N. C.

Patients with multiple myeloma frequently die in uremia. Pathological examination has emphasized blockage of the renal tubules by protein casts, as the cause of the renal failure. If this were true, functional tests should show approximately equal decrease in glomerular and tubular function.

Eight patients with a clinical diagnosis of multiple myeloma, confirmed by $x$-ray, bone biopsy, and other exami- nations, were selected who showed no evidence of other kidney disease. All patients had normal blood pressures and NPN concentrations. The glomerular filtration rate $\left(C_{I N}\right)$, effective renal plasma flow $\left(C_{P A B}\right)$, and tubular secretory maximum ( $T_{m_{P \Delta B}}$ ) were determined, each value being an average of two or more urine collection periods.

In each instance, all three functions were depressed to a lesser or greater degree. In three patients the tubular secretory maximum was much better preserved than glomerular filtration. The severity of renal impairment did not seem to correlate with the hematologic or serum protein abnormality, nor with the amount or duration of Bence Jones proteinuria. The renal functional impairment resulting from hemoglobin- and myoglobin-uria, studied experimentally by Corcoran and Page, showed greatly depressed tubular maxima, compared to the other renal functions, whereas in these patients the converse was the rule.

Tubular obstruction by precipitated protein does not adequately explain the renal impairment frequently found in patients with multiple myeloma. The pathological findings often show relatively little blockage. In one patient who died suddenly without uremia or dehydration, few casts were seen. It seems likely that cast formation may be part of the terminal picture of this disease and not the fundamental basis of the renal failure.

A Comparative Study of the Effects of Tetraethylammonium Chloride and Veratrum Viride on Blood Pressure of Normal and Toxemic Pregnancies. N. S. Assali and Albert A. Brust (Introduced by Eugene B. Ferris), Cincinnati, Ohio.

Humoral maintenance of toxemic hypertension as contrasted with largely neurogenic blood pressure control at term of normal pregnancy has been suggested by observations after autonomic bockade with tetraethylammonium chloride (TEAC). Application of this concept has been further investigated in the present study in which veratrum viride, frequently used in the treatment of toxemia, has been subjected to physiologic evaluation. Ten normal term pregnancies and 12 toxemic pregnancies have been studied with blood pressure assay using intravenous TEAC and veratrum.

Negligible blood pressure response to veratrum occurred at term and postpartum of normal pregnancy, whereas TEAC invariably produced marked blood pressure fall prepartum and minimal responses after delivery.

In toxemia, veratrum lowered systolic and diastolic levels by 50 per cent of the total blood pressure. TEAC administration to the same patients reduced the blood pressure by only 10 per cent. With postpartum recovery from toxemia the marked veratrum response disappeared. As veratrum blood pressure floors rose to levels comparable to those observed postpartum of normal pregnancy, the TEAC floors descended to the same range.

Veratrum side effects (bradycardia, epigastric burning, nausea and vomiting) occurred regularly, but the magnitude of the depressor response was independent of these vagal stimulation components of the drug action. Administration of TEAC at the height of veratrum action in- 
variably abolished the side effects, yet the depressor action persisted.

The data: (1) Demonstrate diametrically opposing blood pressure effects of TEAC and veratrum in both normal pregnancy and toxemia. (2) Eliminate vagal stimulation as the cause of the marked depressor responses to veratrum in toxemia. (3) Indicate that the blood pressure of the toxemic hypertensive is consistently sensitive to veratrum. (4) Offer clues for discussion of possible modes of mediation of veratrum depressor responses in toxemia.

The Problem of Infection of Man with the Virus of Newcastle Disease of Chickens. F. B. BANG and David T. KARzoN (Introduced by Joseph L. Lilienthal Jr.), Baltimore, Md.

From two of three cases of acute conjunctivitis occurring in laboratory personnel working with the virus of Newcastle disease, we have isolated this virus on two occasions each. These individuals did not develop a high titer of neutralizing substance in their fresh sera. In a survey of eight other persons exposed in the laboratory but who had not had conjunctivitis, one was found whose fresh serum neutralizes more than one million lethal infectious doses for the chick embryo. After heating to $56^{\circ}$ C. for one-half hour, this serum loses a large part of its activity, neutralizing less than one thousand lethal doses. When the serum is diluted, there is a very rapid drop in its titer of neutralizing substance. This appears to stand in contrast to a slower fall in titer when the immune serum of chickens convalescent from experimental Newcastle disease is similarly diluted. The substance has been consistently present in fresh samples of serum taken from this person during the past year. No substance of comparable activity has been found in the other sera tested. The nature of the heat labile component is under study.

A Quantitative Analysis of the Effects of Adrenocorticotropic Hormone $(A C T H)$ on Potassium $(K)$ and Phosphorus ( $P$ ) Metabolism. Frederic C. BartTER, Paul Fourman, Anne P. Forbes, William M. JefFRIES and ELEANOR DEMPSEY (Introduced by Fuller Albright), Boston, Mass.

In man, ACTH affects $\mathrm{K}$ and $\mathrm{P}$ metabolism each by three established mechanisms. For $\mathrm{K}$ they are: (1) the loss of protoplasm, (2) the retention of extracellular fluid (e.c.f.), and (3) the specific effect of adrenal "salt" hormone in causing $\mathrm{K}$ loss. For $\mathrm{P}$ they are: (1) the loss of protoplasm, (2) the retention of e.c.f., and (3) the loss of bone (osteoporosis).

Balance studies with ACTH are reported. "Corrections" are applied to the $K$ and $P$ excretions for protoplasm, e.c.f., and bone changes. A fourth effect of ACTH on $K$ and $P$ is thereby unmasked, namely, $K$ and $P$ retention "on" ACTH, and K and P loss "off" ACTH. The deviations of $\mathrm{K}$ and $\mathrm{P}$ so revealed are highly correlated. This fourth mechanism affects $\mathrm{K}$ in the opposite direction to that of the "salt" hormone, and is not elicited by desoxycorticosterone alone.
We infer that this fourth effect represents passage of $\mathrm{K}$ and $P$ into and out of cells, possibly associated with glycogenesis and glycogenolysis, respectively. We suggest that the $\mathrm{K}$ and $\mathrm{P}$ liberated when protoplasm is broken down are retained with glycogen. The net result is a transformation of protein energy to the more readily available glycogen energy.

\section{Apparent Role of Shock in Production of Renal Damage} Accompanying Hepatic Injury. JAMES H. BAXTER (Introduced by Benjamin M. Baker, Jr.), Baltimore, Md.

Rats fed diets containing pyridine regularly developed acute hepatic necrosis; the animals which survived long enough developed cirrhosis. Before death, the animals usually appeared pale and were cold to the touch. The livers of animals autopsied in the terminal stages of necrosis were engorged with dark blood. There was marked obstruction to perfusion of fluid through the livers. Bleeding from the rectum sometimes occurred. Disturbances of hepatorenal vasotropic factors (Shorr), in addition to obstruction and trapping of blood in the liver, may have contributed to the production of shock.

Many of the rats fed pyridine also developed renal injury. Degenerative changes and sometimes necrosis occurred in the convoluted tubules. Regenerative changes followed, with the formation of cellular buds or masses in the region of the straight segments. Generalized or localized dilatation of tubules with flattening of epithelium was regularly observed, and areas of cortical scarring and atrophy resulted when length of survival permitted.

Liver injury frequently occurred in animals without kidney injury. However, kidney injury was seldom observed prior to the occurrence of extensive liver injury, even though some animals remained on the diets for weeks without developing liver injury. Furthermore, the most extensive renal injury occurred in animals which remained in shock for considerable periods or which survived recurrent episodes of shock. It appeared likely that the renal injury was at least in part due to shock, resulting in impairment of cortical circulation with anoxia of tubular cells, oliguria, and increased concentrations of protein in the urine. Similar mechanisms may participate in the production of renal injury under a wide variety of circumstances.

\section{Preliminary Studies on an Indirect Method for Determin- ing Portal Pressures. William B. Bean and (by invi- tation) William D. Paul and Murray Franklin, Iowa City, Iowa.}

There are circumstances in which it is desirable to know whether or not pressure in the portal vein is elevated. Previous observations on portal pressures have been confined to experimental animals except for a few direct measurements obtained during abdominal operations. We have approached the problem in normal persons and those with clinical signs of cirrhosis of the liver by observing through the proctoscope or sigmoidoscope changes in color of the mucosal surfaces with measured increments of intraluminal pressure. 
The escape of air upwards in the colon is prevented by inserting an inflatable rubber bag attached to a tube. The sigmoidoscope is connected with a water manometer and by $T$-tube to a rubber bulb pump and small air reservoir so that air pressure within the rectum and sigmoid can be varied at will and measured readily. Subjects are prepared by cleansing enemas. The rubber bag is inserted, the proctoscope introduced and the bag is inflated. The glass window with adaptor for communicating with the manometer and pressure reservoir are adjusted. With the patient in the left lateral (Sim's) position, the anatomical center of the liver is approximately on the same level as the observed mucosa. Pressure must be increased slowly to reduce intestinal movements and cramps. At levels between 10 and $20 \mathrm{~cm}$. of water, the pink color fades and many vessels are seen against the pale background. Between 40 and $60 \mathrm{~cm}$. the caliber of the vessels narrows. With manual pressure over the liver, the level at which blanching occurs is elevated to $30 \mathrm{~cm}$. or more. Similar levels have been noted in persons with portal hypertension and cirrhosis. The method may be useful in evaluating pain thresholds in the lower bowel and the acute effects of drugs.

Myofilaments and Myofibrils of Cardiac Muscle. $\mathrm{H}$. Stanley Bennetr, Seattle, Wash.

Electron micrographs have been obtained of frog cardiac muscle elements fixed in formaldehyde or trichloracetic acid and fragmented with blender and homogenizer. The preparations have yielded pictures of myofibrils, which prove to be packed with parallel myofilaments similar to those found in striated skeletal myofibrils by Hall, Jakus, and Schmitt. The cardiac muscle myofilaments are slightly thicker than those of skeletal muscle, averaging 240-260 A in diameter, and show an axial repeating period of about $400 \mathrm{~A}$ when unstretched. As in skeletal muscle, the filaments appear to be indefinitely long, extending through many sarcomeres. Evidence of fine structure within the $400 \mathrm{~A}$ period can be seen, but the material does not permit definitive analysis of such details. The filaments are capable of being stretched readily and fortuitously during fragmentation or on the supporting film, so that periodicities from $375 \mathrm{~A}$ to about $750 \mathrm{~A}$ may be measured, depending on the degree of stretching. Electron micrographs of cardiac muscle myofibrils show the various bands visible with the light microscope, but the bands are less distinct than in skeletal muscle. The Z-band is about $0.15 \mu$ wide in cardiac muscle.

The Effect of a Cation Exchange Resin on Electrolyte Balance and Its Use in Edematous States. Eugene Y. Berger, Leslie Irwin, Benjamin Rosenberg and RosLYN Jackenthal (Introduced by Alfred E. Cohn), New York, N. Y.

An exchange resin, Liquonex $\mathrm{CRW}$, which removes cations from solution in exchange for hydrogen was fed to a subject on a constant diet. An electrolyte balance study was conducted for three weeks before, five weeks during, and three weeks following the administration of the resin. Approximately 80 per cent of dietary sodium and 70 per cent of dietary potassium appeared in the stool. There was no evidence of a calcium deficiency. A compensated acidosis was induced by the liberation of hydrogen ions in exchange for sodium ions. The excess chloride, phosphate, and sulphate anions were balanced in the kidneys by formation of ammonia.

Fixation of sodium in the gut is equivalent to restricting sodium in the diet; hydrogen ion release is the equivalent of giving ammonium chloride or an acid ash diet. Both of these procedures are commonly used to help prevent edema formation. Therefore, two patients suffering from heart failure and edema and one from cirrhosis of the liver and ascites were fed the resin. In the two cases of congestive heart failure on a diet containing 6 grams of salt daily, the use of resin replaced the need for mercurial diuretics and salt restriction. In the patient with cirrhosis of the liver on a similar diet, the ascitic fluid disappeared in 22 days without the use of mercurial diuretics. A well marked but still compensated acidosis was produced in the normal individual and in the patient suffering from cirrhosis of the liver. These observations indicate that a cation exchange resin can restrict the absorption of sodium from the intestine. Use of the resin may therefore become important whenever it is desirable to maintain a high protein low sodium diet.

Studies in the Renal Mechanisms for the Excretion of Potassium. ROBERT W. BerLINER, Thomas J. KenNEDY, JR. and James G. Hilton (Introduced by A. B. Gutman), New York, N. Y.

When large amounts of potassium salts are administered to normal dogs by constant intravenous infusion, the amount excreted in the urine may exceed that filtered by as much as 80 per cent. Exact reproduction of such experiments is mechanically difficult in humans, but potassium clearances greater than glomerular filtration rate have been observed in normal man, and in normal and hypertensive man on the rice diet.

The coexistence of a secretory mechanism for excretion of a substance ordinarily filtered and reabsorbed has not previously been observed. The paradoxical behavior of $\mathrm{K}^{+}$, capable of moving across the tubular epithelium in opposite directions, makes evaluation of the relative magnitude of the reabsorptive and secretory mechanisms difficult since only the resultant can be examined. Efforts to evaluate the roles of these two opposing mechanisms have been made chiefly in dogs.

Chronic administration of moderate doses of potassium by inducing tolerance, facilitates excretion of sudden large loads of this ion at more rapid rates and at lower plasma concentrations than in unprepared dogs.

Infusion of potassium with non-reabsorbable anions such as ferrocyanide or thiosulfate regularly results in ratios of excreted to filtered higher than with reabsorbable chloride. Moreover, in such experiments, the minimal secreted potassium is so much larger than the anions which could possibly have been secreted that wation exchange must have occurred. Hypothetical mechanisms, the overall result of which is ionic exchange, are discussed. 
When infused sodium thiosulfate or ferrocyanide is progressively replaced by the corresponding potassium salt, change from net reabsorption to net secretion of potassium is first associated with increased excretion of chloride and bicarbonate, and, later, reduced excretion of sodium. This probably indicates that excretion of large amounts of potassium is initiated by depression of reabsorption and enhanced by cation exchange.

Studies in Hypothermia: Part I-Oxygen Consumption, Transport and Utilization in Dogs at Low Body Temperature. W. G. BIGELOW, W. K. LiNDSAY, R. C. HARRISON, R. A. Gordon and W. F. GREenwoOd (Introduced by James A. Dauphinee), Toronto, Canada.

This is the report of the first of a series of studies dealing with the physiological effects produced in dogs by general hypothermia.

Twenty-seven mongrel dogs were cooled to body temperatures of $20^{\circ} \mathrm{C}$. or lower by being placed in a controlled temperature room at $-10^{\circ} \mathrm{C}$. Oxygen consumption was determined in the first experiments by analysis of expired air collected in a Douglas bag and in the later ones by the use of a modified Tissot spirometer. Arterial and venous oxygen saturations and carbon dioxide contents were measured at intervals. The dogs were revived by placing them in a water bath at $42^{\circ} \mathrm{C}$.

One hundred and twenty valid determinations of oxygen consumption during the process of cooling and rewarming are reported. Early in the study it was found essential to eliminate all shivering and to maintain normal arterial oxygen consumption. Anesthesia was used to accomplish the former, artificial respiration the latter. Oxygen consumption was found to decrease steadily and in a relatively constant manner at the rate of 5 per cent per degree Centrigrade of temperature lowering, and to increase at a similar rate during rewarming.

The determination of arterio-venous oxygen differences revealed that, at least down to $18^{\circ} \mathrm{C}$. and in the presence of a satisfactory heart action, the circulation in these cooled dogs was adequate to transport oxygen to the tissues.

The finding that the increase of oxygen consumption on rewarming paralleled the decrease on cooling, together with the fact that the general deportment and response of the dogs that survived showed no evidence of tissue hypoxia, indicates that tissues in the cold state are capable of oxygen utilization.

Generalized hypothermia under the conditions of this study reduces tissue oxygen consumption without producing significant tissue hypoxia.

Coronary Blood Flow, Cardiac Oxygen Consumption and Efficiency in Man. R. J. Bing, M. Hammond, J. Handelsman, S. R. Powers, F. Spencer, J. Eckenhoff, W. Goodale, J. Hafkenschiel and S. Kety (Introduced by Alfred Blalock), Baltimore, Md. and Philadelphia, Pa.

The coronary blood flow and oxygen consumption per 100 grams of left ventricular tissue have been studied in
30 individuals, by combining the nitrous oxide method with coronary sinus catheterization. Left ventricular efficiency was calculated for normal heart muscle. In hypertrophy, only low values for efficiency can be considered significant, since total left ventricular weight cannot be estimated. Tests were performed on normal individuals, and on patients with anemia, essential hypertension and coarctation. Four patients in failure were also studied.

In the normal, left ventricular flow averaged $65 \mathrm{cc} . / 100$ grams/ minute, left ventricular oxygen extraction 12 vols. per cent and left ventricular oxygen consumption $7.8 \mathrm{cc}$. /100 grams/ minute. The normal left ventricular effciency averaged 23 per cent. In anemia, the coronary flow was increased, left ventricular extraction and oxygen consumption $/ 100 \mathrm{grams} /$ minute were reduced. In essential hypertension coronary flow/100 grams/minute, left ventricular oxygen extraction and oxygen consumption $/ 100$ grams were normal. In coarctation coronary flow $/ 100$ grams/minute, AV difference and oxygen consumption $/ 100 \mathrm{grams} / \mathrm{minute}$ were increased. In failure the coronary flow/100 grams/minute was reduced and the oxygen extraction slightly increased. The oxygen consumption/100 grams/minute was normal. These findings indicate that 1 , chronic increase in load results in no change in oxygen consumption per unit weight but in a total increase in oxygen consumption due to hypertrophy. 2 , The cardiac efficiency in failure is low.

Characteristics of Visceral Sensation in Man, as Observed in Normal Subjects and Patients with Unilateral Sympathectomy. J. R. Bingham (by invitation), F. J. INGELFINGER and (by invitation) R. H. SMITHWICK, Boston, Mass.

Deep somatic pain in man can be differentiated from abdominal visceral pain. In 5 subjects, deep somatic pain was produced by injecting the intraspinous ligaments and the rectus abdominis with hypertonic saline. Before, during, and after deep somatic pain so produced, a balloon was distended in various parts of the duodenum and jejunum. The two types of pain were perceived to be clearly different. These observations do not support the statement of Lewis that deep somatic and abdominal visceral pain are identical.

After unilateral sympathectomy, pain produced by distending the gut is referred to the unoperated side. This observation was unequivocal in the 10 patients studied. The degree of lateralization appeared to vary with the site of stimulation. Greatest lateralization occurred with duodenal or biliary tract pain, less with high jejunal, and least with low jejunal or high ileal pain. This observation may explain the contradictory findings of others.

After unilateral sympathectomy, the pain threshold is elevated. In 4 patients ( 2 subjected to intestinal, and 2 to biliary tract distention), almost double the amount of pain stimulus was required after, as was needed before sympathectomy. In 5 other patients, studied only post-operatively, the pain threshold was elevated as compared with the normal average. This observation may explain why unilateral sympathectomy sometimes relieves visceral pain arising from a bilaterally innervated organ. 
The common duct is bilaterally innervated. Distention of the common duct produced pain in one patient with a right unilateral, and in one patient with a left unilateral sympathectomy. This observation, confirming Ray's, emphasizes that complete desensitization of the biliary tract cannot be achieved by right sympathectomy.

Metabolic Fate of Infused Erythrocytes in Man. F. Ross Birkhill, Mary A. Maloney and Stanley M. LevensoN (Introduced by John B. Youmans), Chicago, Ill.

The metabolic fate of infused erythrocytes in adults made significantly polycythemic by transfusions has not been known. Only inadequate and incomplete information has been available on erythrocyte survival and bone marrow function in such individuals. Data on these points were obtained in two normal adult males on a constant diet receiving serologically indentifiable, fresh, separated erythrocytes. Observations were made on body weight, nitrogen, potassium, and phosphorus balances; circulating plasma protein and erythrocyte mass; erythrocyte survival; sternal bone marrow; reticulocyte counts; serum bilirubin concentration; urobilinogen excretion; and liver function.

Survival time of the infused erythrocytes was not shortened. The infused red cell mass decreased at a normal, expected rate of 0.8 per cent per day. Concomitantly, the mass of the recipient's own erythrocytes declined at a rate of 0.4 to 0.8 per cent per day in direct proportion to the amount of the infusions. This progressive fall in the subject's own erythrocyte mass was probably due to erythropoietic depression rather than to abnormally increased destruction. This is suggested by the normal survival of the infused erythrocytes and by urobilinogen excretion consistent with breakdown of the total red cell mass at a normal, not an increased, rate. There was a direct linear relationship between the extent of bone marrow depression and the degree of induced polycythemia.

A slow, steady excretion of 0.5 to 1.0 grams of nitrogen per day (above the control equilibrium value) began shortly after the infusions and continued for one month. The total extra nitrogen excreted was roughly equal to the total nitrogen content of the infused erythrocytes. However, this excess nitrogen derived only in small part from the infused red cells. The greater part could be accounted for almost completely by nitrogen diverted from normal erythrocyte synthesis as a result of marrow depression.

The Effect of Increased Intra-Abdominal Pressure Upon Renal Excretion of Water, Sodium and Potassium in Normal Human Subjects and in Patients with Diabetes Insipidus. S. E. BradLEY, and (by invitation) G. H. Mudge, W. D. Blake and P. Alphonse, New York, N. $Y$.

Elevation of intra-abdominal pressure in man by application of a pneumatic girdle under a pressure of $80 \mathrm{~mm}$. $\mathrm{Hg}$ has been shown to depress glomerular filtration, renal blood flow, and maximal tubular glucose reabsorption and diodrast excretion. The output of urine is greatly reduced and the urinary concentrations of such substances as mannitol and inulin increase. Further studies were undertaken to assess the role of the antidiuretic hormone of the pituitary in this phenomenon and to determine whether electrolyte retention also occurred.

Mannitol, p-aminohippurate ( $\mathrm{PAH})$, sodium, and potassium clearances were determined simultaneously in six normal human subjects and in five patients with uncontrolled diabetes insipidus before, during, and after abdominal compression. In all but in two normal subjects and one patient with diabetes insipidus, glomerular filtration (as mannitol clearance) and renal blood flow (PAH clearance) decreased significantly, and the excretion of sodium, potassium, and water was regularly reduced. These responses were identical in both groups of subjects. Sodium and water output usually fell more than filtration, whereas changes in potassium excretion appeared to parallel changes in filtration. In most instances, the urinary concentration of sodium decreased during compression. All values tended to return toward the control levels following release of pressure. Increased intra-abdominal pressure appears to induce a renal retention of water and electrolytes primarily as a result of a reduction in glomerular filtration, independently of the production of antidiuretic hormone. Augmented tubular re-absorption of water and sodium may account for disparities between changes in filtration and in sodium and water excretion observed in some subjects.

\section{Tetralogy of Anomalous Pulmonary Drainage, Auricular Septal Defect, Right Ventricular Hypertrophy and Pat- ent Ductus Arteriosus. Robert A. BrUce, Frank W. Lovejoy, Earle B. Mahoney, George B. Brothers, Paul N. G. Yu and Raymond Pearson (Introduced by Nolan L. Kaltreider), Rochester, N. Y.}

Since patent ductus arteriosus is usually amenable to surgery, and ligation of an anomalous pulmonary vein has been recommended, the combination of both lesions together with auricular septal defect and right ventricular hypertrophy presents a complicated clinical problem. Two out of 12 possible patients reported in the literature lived for 19 and 27 years. We have studied 2 cyanotic young women, one of whom was proved to have this tetralogy at autopsy, and the other had similar findings by catheterization. The first had frequent major hemoptyses for which she was subjected to ligation of an anomalous right pulmonary vein demonstrated by catheterization. She died of pulmonary edema, immediately following ligation of the anomalous vein. The second had marked clubbing but no hemoptyses. The $\mathrm{x}$-ray findings in both were a relatively small heart despite enlargement of the right auricle and prominence of the pulmonary conus and of vascular markings in the lungs. Each had right axis deviation electrocardiographically. Exercise studies showed transient paradoxical response in the respiratory efficiency attributed to the shunts. The right ventricular pressure considerably exceeded the systemic pressure in both patients, and in one catheterization of the left ventricle demonstrated that the duration of systole was less than that of either the right ventricle or systemic pulse. This was in accord with Brody's hypothesis that the right ventricle 
could supply a major part of the systemic circulation through the ductus. Our experience shows that cyanosis contra-indicates ligation of an anomalous pulmonary vein whenever it is demonstrated to be draining into the right heart and that in this tetralogy patency of the ductus is essential. Slides of the laboratory data, anatomical findings in the first patient, and cinefluorograms (courtesy of Dr. Sibley Watson) of both are shown to demonstrate this particular tetralogy of congenital cyanotic heart disease.

The Lack of Correlation between Glomerular Filtration Rate, and Serum Electrolyte Concentration Changes, Urinary Electrolyte Excretion, or Edema Formation Following Sodium Loads in Subjects with Normal Kidneys, Glomerulonephritis, and the Nephrotic Syndrome. Charles H. Burnett, (by invitation) Belton A. BurROws and (by invitation) ROBERT R. COMMONS, Boston, Mass.

The effects of sodium loads administered intravenously to subjects with normal renal function, and with glomerulonephritis in different stages were studied. Normal responses to 200 to 400 milliequivalents of sodium (as 0.5 $M$ sodium bicarbonate) included: serum concentrations, sodium increased, potassium slightly but definitely decreased; urinary excretion, sodium and potassium increased; percentage excretion of filtered ion (calculated from $\mathrm{C}_{\mathrm{in}}$ ), sodium slightly and potassium markedly increased; ratio of reabsorbate concentration to serum concentration (R/S), sodium remained close to unity, potassium fell (as much as 25 per cent). The direction of these changes was consistent despite individual variations in filtration rate and urine flow.

A different sequence resulted in the nephrotic syndrome, with normal or only moderately low glomerular filtration rates: sodium rose or was unchanged in the serum, urinary excretion failed to increase, percentage excretion of filtrate remained low, but $R_{\mathrm{Na}_{2}} / \mathrm{S}_{\mathrm{Na}}$ was unchanged. Further edema always resulted. With one exception, the effects on potassium metabolism were identical with, or more marked than, those occurring in normals.

In glomerulonephritis, without edema or heart failure, both the normal and nephrotic responses occurred. Ability to excrete the sodium load diminished as $\mathrm{C}_{1 \mathrm{n}}$ fell; but excess sodium was better excreted than in nephrosis, and edema never resulted. The potassium responses were accentuated in that percentage excretion of filtrate often exceeded 100 (suggesting renal tubular secretion of this ion).

It is concluded, that since the rate of sodium excretion could not be correlated uniformly with either the glomerular filtration rate or tubular reabsorption of sodium, the mechanism of edema formation remains undisclosed in the diseases studied. It is suggested that the almost invariable change in potassium metabolism resulting from sodium administration may represent an important factor in the maintenance of salt and water equilibrium.

The Incidence of Liver Involvement in Chronic Amoebiasis. RICHARD B. CAPPS and (by invitation) ALFRED Bennett, Chicago, Ill.
The incidence of liver involvement in chronic amoebiasis is of interest both because of its bearing on treatment and also because of its role in the production of symptoms. In order to provide both a sensitive and a specific method of establishing the presence of amoebic hepatitis, the response to emetine of a group of liver function tests was studied. Observations were made on a series of 15 consecutive cases of chronic amoebiasis. All cases had symptoms but in no instance was fever present. Liver function tests including the cephalin cholesterol flocculation, thymol turbidity, serum alkaline phosphatase, serum bilirubin, bromsulphalein, 24 hour urine urobilinogen and urine coproporphyrin were performed before and after a course of emetine. In 11 cases the initial tests indicated mild liver dysfunction. Following emetine the abnormal findings returned to normal promptly or dropped sharply in almost all instances indicating a specific therapeutic response. In 10 of these 11 cases liver tenderness and symptoms consistent with hepatitis were present initially and also responded to emetine.

We believe that these findings indicate that a specific hepatitis is present in a large percentage of cases of chronic amoebiasis and is responsible for many of the symptoms. Unless other agents can be shown to be effective emetine should probably be used routinely in all cases of amoebiasis. Further studies are being conducted.

The Golgi Apparatus in the Cells of Synovial Tissue and Related Structures. Pedro M. Catoggio and L. RayMOND MORRISON (Introduced by Charles L. Short), Boston, Mass.

A study was made of the relationship between the size, configuration and localization of the Golgi element and the histological findings in tissue from joints, bursae and ganglia removed at operation from seventy-five patients. In some instances the relationship to the joint fluid was also studied.

The material was prepared according to the Da Fano technique. Tissue from some of the cases was also studied by other methods.

The clinical diagnoses were: normal joints, acute traumatic arthritis, chronic traumatic arthritis and degenerative joint disease, rheumatoid arthritis, miscellaneous joint disease, bursitis, ganglion.

Examination of the sections showed a well-developed Golgi element in the synovial cells. In some cases it was small, granular or simple and delicately looped and was often indifferently located within the cytoplasm of small cells; presumably in the "resting state." This appearance was usually associated with apparently normal synovial tissue. In other instances the Golgi apparatus was large, complex and lay in relation to the free border of the cell and was presumably "active." These specimens showed various synovial tissue alterations: increased number of synovial cells, larger cells, villi and often perivascular round-cell infiltration.

If increase in size and complexity of the Golgi apparatus is evidence of increased activity of the cells, the above findings suggest that the synovial cells in rheumatoid arthritis are often more active than in normal 
joints. In degenerative joint disease the activity of these cells appears to be between that in rheumatoid arthritis and that in normal joints.

In the small number of joint fluids available, comparison of the fluid characteristics with the appearance of the Golgi element in the tissue cells does not provide adequate evidence to aid in determining whether or not synovial cells produce mucin.

Simultaneous Determination of Cardiac Output by the Acetylene and Direct Fick (Right Heart Catheterization) Procedures. Carleton B. Chapman, Henry L. Taylor, Craig Borden, Austin Henschel, and Ancel Keys (by invitation) and Richard V. EBerT, Minneapolis, Minn.

Nineteen simultaneous measurements of the cardiac output by the acetylene and direct Fick (right heart catheterization) techniques were carried out on 7 normal subjects. The Grollman formula was used for acetylene determinations. The mean cardiac indexes were $2.47 \pm 0.56$ (acetylene), and $3.27 \pm 0.78$ (direct Fick) liters per square meter per minute. When plotted against each other, the results obtained by the 2 methods showed a definitely linear relationship $(r=0.87)$. Statistical analysis showed that the small deviation from a perfectly linear relationship was due to individual method errors. If the acetylene output $(x)$ is known, the direct Fick output (y) can be calculated from the formula $y=1.1682 x+$ 0.7420 , the standard error of the estimate being \pm 0.41 . The Grollman formula, therefore, can be made to yield results which closely approximate those obtained by the direct Fick procedure simply by altering the numerical constant.

In order to determine how much, if any, of the predictable difference between results of the 2 methods is due to recirculation of acetylene, 30 samples of pulmonary arterial blood were taken at from 9 to 55 seconds after acetylene entered the pulmonary alveoli. When the partial pressure of acetylene in the blood was plotted against time, recirculation began between 10 and 20 seconds after beginning of rebreathing and progressed in a linear fashion. For the interval between the first and second gas samples ( 9 to 19 seconds), the calculated mean partial pressure of acetylene in pulmonary arterial blood was $3.5 \mathrm{~mm}$. $\mathrm{Hg}$ or about 5 per cent of the mean partial pressure of acetylene in the alveoli $(61.5 \mathrm{~mm}$. $\mathrm{Hg})$. This confirms the view that such recirculation as occurs during the first 20 seconds after acetylene enters the alveoli is not enough seriously to affect the validity of the method.

Some Cardiac and Extracardiac Effects of Digitalis Glycosides. Don W. Chapman and Carroll A. Handeley (Introduced by James A. Greene), Houston, Texas.

Studies were undertaken to ascertain what relationship, if any, existed between the intravenous administration of digitalis glycosides and the serum concentrations of potassium and sodium and their effect on the electrocardiographic tracings; hematocrite; the plasma volume; and the extracellular fluid.
Digitoxin and digoxin were employed in concentrations from 0.1 to $0.2 \mathrm{mg} . / \mathrm{kg}$. body weight in twenty-seven dogs. Serial electrocardiograms were taken from lead 2 and blood samples were drawn at hourly intervals for the estimation of serum potassium and sodium. Sodium thiocyanate and T1824 were employed to ascertain changes in extracellular fluid and plasma volume at four to ten-hour intervals. Hematocrite determination was by the Wintrobe method.

When digitalis was administered in small doses so that relatively minor or no electrocardiographic effects were obtained, in general, there was but little change in the other studies. In larger doses an approximate qualitative relationship between the increasing height of the serum potassium and the electrocardiographic alterations, as seen in potassium intoxication, were noted. The initial changes observed were an increase in the amplitude of the $T$ waves, followed by a decreased size of the $R$ waves, and an increased amplitude of the $S$ wave. In some, there was a disappearance of $R$ waves with a depressed RST segment or a widening of the QRS complex. In others a partial or complete auricular arrest may have preceded the development of intraventricular block. Ectopic rhythms such as single or multiple premature ventricular contractions may be seen. However, in some instances, no direct correlation was noted with the height of the serum potassium. Marked increases in the hematocrite, a lowering of the plasma volume, and an increase in the extracellular fluid was observed when there were marked changes in the electrocardiographic tracings.

\section{Multiple-Balloon-Kymographic Recording of Variations in Motility of the Upper Small Intestinal Tract in Man, Emphasizing the Need of Long Control Observations for the Adequate Evaluation of Drug Action. WIL- liam P. Chapman, (by invitation) Eirwyn N. Row- Lands, and Chester M. Jones, Boston, Mass.}

Knowledge of spontaneous variations in intestinal motility in healthy subjects observed over long periods was regarded as essential for the evaluation of drugs, particularly when given orally. Fourteen studies averaging four hours in duration were made in twelve subjects, all except one being healthy young adults. Motility was recorded from the duodenum and upper jejunum using a four-lumen tube with balloons spaced at four-inch intervals. Placebos without verbal suggestion were administered on an average of fifty-one minutes after the start of the recording. Relative changes in contractions and "tone" or sustained tension of the intestine were assessed by visual inspection of the records and by polar planimeter measurements of surface areas beneath the tracings.

With individual exceptions, the group showed during the second, third, and fourth hours of observation a decline in peristaltic contractions fluctuating from thirty to thirty-seven per cent. Sudden, striking decreases in activity lasting from five to twenty minutes followed the spontaneous occurrence of spasms. Segmental waves, though difficult to assess, seemed to follow the course of peristaltic waves. "Tone" varied with alterations in the types of contractions and tended to increase slightly 
with the passage of time. Most individuals showed a specificity of pattern as regards frequency, amplitude and duration of contractions and "tone."

Without a full appreciation of the spontaneous decline in contractions during long observation periods, the tendency of contractions to be temporarily abolished or decreased following spasms and individual motility variations, it is impossible to evaluate adequately the pharmacologic effects of drugs on intestinal motility. This is particularly true of drugs administered orally. Multiple balloon recording permitted simultaneous assessment of adjacent intestinal segments. Changes in motility appeared not to be conditioned by the administration of placebo as employed in this study. Infrequency of pain prevented its correlation with motility patterns.

\section{Effects of Salt and Protein Restriction on Blood Pressure and Renal Hemodynamics in Hypertensive Patients. H. Chasis and (by invitation) W. Goldring, E. BrEed, A. Bolomey and H. W. Smith, New York, N. Y.}

Twelve patients with essential hypertension were selected at random. Observations were made on blood pressure, glomerular filtration rate, renal blood flow and maximal tubular excretory capacity. The patients were maintained on a salt and protein restricted diet identical in composition with the rice diet suggested by Kempner, for from 15 to 98 days. Renal function measurements were made in 10 of the patients; in 9 the rate of glomerular filtration was reduced, in 8 the renal blood flow was decreased, and in 6 the maximal tubular excretory capacity was reduced. In 10 of the 12 patients no lowering of blood pressure was effected by the restricted diet used in this study. In the remaining two patients decrease in blood pressure levels of 24 and $22 \mathrm{~mm}$. $\mathrm{Hg}$ systolic and 14 and $14 \mathrm{~mm}$. $\mathrm{Hg}$ diastolic occurred. However, in view of the spontaneous variability of blood pressure levels, crudity of the sphygmomanometric method and difficulty in selection of a datum of reference during the control period, we are unwilling to accept as significant such relatively slight and transient reductions as indication of effectiveness of the dietary regime employed in this study.

In view of the adverse effects observed on renal hemodynamics and the transient and insignificant reduction of blood pressure we conclude that salt and protein restriction was ineffective therapy in the 12 hypertensive patients studied.

Studies on the Initiation of Coagulation by Surface Contact. C. Lockard Conley, Robert C. Hartmann and John S. Lalley (Introduced by G. Canby Robinson), Baltimore, Md.

Using silicone-treated apparatus blood was obtained from normal human subjects without the use of anticoagulants. The formed elements were removed by high-speed centrifugation at low temperatures. Direct microscopic examination of the undiluted plasma revealed as a rule less than 10 platelets per cubic millimeter. In some instances no platelets were seen. Plasmas prepared in this manner regularly clotted within a few minutes at $37^{\circ} \mathrm{C}$. following the addition of ground glass. Platelet extracts prepared by grinding washed platelets in glass containers also brought about coagulation. However, the concentrated platelet extracts did not cause coagulation to occur more rapidly than did ground glass when added to these essentially platelet-free plasmas. The rate of clot formation following the addition of ground glass was related to the amount of glass added and was independent of the number of platelets or quantity of platelet extract present. The amount of prothrombin converted during coagulation seemed directly related to the amount of platelet material available. When plasma was clotted by ground glass in the virtual absence of platelets, prothrombin consumption seemed negligible. Unlike normal plasma, hemophilic plasma deprived of its platelets failed to clot on contact with glass.

Normal platelet-deficient plasma could be kept in silicone-treated tubes at $37^{\circ} \mathrm{C}$. for relatively long periods of time without spontaneous coagulation even though clotting occurred promptly following the addition of ground glass. Although fresh normal plasma invariably clotted on contact with glass, portions of this plasma stored for 24 hours at $2^{\circ} \mathrm{C}$. in some instances failed to clot on the addition of ground glass.

These observations suggest that the initiation of coagulation by surface contact may be brought about by alterations produced in the plasma itself and not necessarily by platelet changes.

Effects of Intravenous Reduced Glutathione (GSH) Upon Glycosuria and Hyperglycemia Induced in Man by Adrenocorticotropic Hormone ( $A C T H$ ). JEROME W. CoNn and (by invitation) LAWRENCE H. Lours and Margaret W. Johnston, Ann Arbor, Mich.

We have reported that sustained glycosuria and hyperglycemia can be produced in man by administration of ACTH and that these manifestations of a developing diabetic state are accompanied by a decreasing concentration of blood glutathione. We have hypothesized that a purine metabolite having an alloxan-like effect is responsible for reducing the intracellular availability of free sulfhydryl groups (necessary for normal function of many enzyme systems), causing functional abnormalities within the islet-beta-cells as well as blocking normal glucose utilization peripherally. In addition to hyperglycemia, ACTH decreases renal tubular reabsorption of glucose, presumably as the result of inhibition of an enzyme within the tubular epethelium.

A thirty day metabolic balance study was made to determine whether or not administration of large amounts of GSH would reverse (during continued ACTH administration) an already established hyperglycemia and glycosuria produced in a normal man. GSH in large amounts was given on the fourth day of a six day ACTH period. Renal glycosuria occurred on the first day of ACTH. Hyperglycemia began on the second day and continued with increasing intensity throughout the entire ACTH period except during the interval when GSH was administered. This reversal was dramatic but transitory, lasting for one to two hours after each injection of GSH. 
During these periods the urine was free of sugar. Most striking were (1) sharp elevation of the renal threshold and (2) a fall of the blood sugar level.

Not all of the metabolic effects of ACTH were reversed by GSH. This means that corticoid production was not blocked but that improved performance occurred in those systems requiring free $\mathrm{S}-\mathrm{H}$ groups for their normal function. Among other reversed effects noted following GSH was a most profound change in the number and character and the peripheral blood cells.

All of these sharp reversals produced by GSH during ACTH administration strengthen our hypothesis and imply important hormonal influences upon enzyme activity.

Observations on the Hemolytic Mechanism in Paroxysmal Nocturnal Hemoglobinuria. William B. CrosBy (Introduced by William Dameshek), Boston, Mass.

The red cells in paroxysmal nocturnal hemoglobinuria (PNH) are easily hemolyzed by the action of a heatlabile substance present in normal human plasma. This substance is similar in many respects to complement. Like complement, its hemolytic activity is blocked by heparin, although the amount of heparin needed for this inhibition is somewhat less than that needed to inhibit complement. Titers of $1: 4,000$ will almost completely block the hemolytic system of $\mathrm{PNH}$ in vitro.

Protamine, a potent heparin antagonist, prevents this heparin inhibition. Yet alone in the hemolytic system, protamine acts like heparin and inhibits hemolysis.

Titration with protamine or heparin in the PNH hemolytic system yields in each case, a sigmoid curve which breaks upward at about $1: 3,000$ and levels off at about $1: 8,000$. When cell suspensions containing the two antagonists in equal concentration are mixed, protamine seems much the stronger. Four parts of heparin cause no inhibition of hemolysis in the presence of one part of protamine.

A patient with $\mathrm{PNH}$ was given intravenously fifty $\mathrm{mg}$. of heparin. This at once reduced the ability of his plasma to hemolyze his cells when incubated in vitro. During the ensuing six hours the hemolytic potency of his plasma gradually recovered. To a second patient, $80 \mathrm{mg}$. of heparin were given at the time of a transfusion in the hope of preventing a transfusion reaction to which she was prone. But a violent hemolytic reaction followed. In both of these patients the hemolytic mechanism was blocked in vitro by heparin.

Protamine and heparin are antagonistic by virtue of powerful and opposed electrostatic charges. Each alone is capable of inhibiting hemolysis in the hemolytic system in PNH. When both are present together in the hemolytic system they inhibit one another, and hemolysis occurs. Their effect is probably due to inhibition of a complement-like substance present in normal human plasma.

Primary Aseptic Meningitis: A Clinical Syndrome of Diverse Etiology. EDWARD C. CURNEN and (by invitation) Josepr L. Melnick, and Ernest W. Shaw, New Haven, Conn.
During the summer of 1948 a number of children and young adults were admitted to hospitals in Connecticut and Rhode Island with the clinical syndrome of primary aseptic meningitis. In most instances a diagnosis of poliomyelitis was made. These cases considerably exceeded in number those with paralytic poliomyelitis. The clinical and laboratory findings were studied in selected patients with representative non-paralytic illnesses.

Prominent initial symptoms were fever, headache, and vomiting. Nuchal rigidity and stiffness of the back were common but transitory complaints. The leukocyte counts approximated normal. The cerebrospinal fluid revealed a pleocytosis, usually less than 500 and predominantly lymphocytic; the protein content was normal or slightly elevated.

The course of the illness was brief and uncomplicated. All patients recovered completely. In patients with this syndrome, differentiation of non-paralytic poliomyelitis from mild non-paralytic illnesses due to other causes usually could not be made from clinical findings alone.

Attempts were made to recover the causative agent from representative patients with primary aseptic meningitis. Suspensions of stool from 2 of 14 patients tested induced poliomyelitis in monkeys. Similar suspensions injected into suckling mice resulted in the recovery of a filterable agent resembling that reported by Dalldorf and Sickles. Separate strains were obtained from 5 patients. Attempts to recover this agent from 9 other patients with aseptic meningitis (including the 2 whose stools induced poliomyelitis in monkeys), from 2 patients who developed paralytic poliomyelitis in 1948 in Connecticut, and from 20 patients with other illnesses were unsuccessful. Further efforts are in progress to characterize this agent, to correlate its occurrence with clinical and serological findings, and to determine whether infection by it represents a distinct disease entity.

Physiologic, Metabolic, and Electrolytic Balance Studies of Hypertensive Patients While on the Rice Diet. H. Currens, E. A. S. Reid, E. A. Maclachlan and M. L. TerRy (by invitation) and A. M. BUtLer and P. D. White, Boston, Mass.

In an effort to determine the modus operandi of the rice diet two men (one had previously undergone sympathectomy) were studied before and after instituting dietary therapy. The following pertinent observations were made.

The blood pressure fell in one patient after two weeks $(174 / 126$ to $160 / 111)$ and the other after three months (166/112 to $141 / 87)$ on the diet. The addition of nine grams of salt per day to the latter resulted in a rise $(164 / 103)$.

A negative balance was observed for sodium, chloride, calcium, phosphorus, and magnesium on the diet. One patient had a positive potassium balance of about 20 milliequivalents per day. The serum concentration of these electrolytes remained essentially the same. The negative nitrogen balance was approximately five grams per day during the first week on the diet decreasing to one gram 
after three months. The serum albumin fell significantly after three months.

An abrupt fall of the basal metabolic rate of about 12 points was observed after beginning the diet. No significant weight loss was noted. The insensible weight loss per day remained the same.

The plasma volume as measured by the Evans blue dye before and after the institution of the diet was found to remain constant.

Renal clearance tests revealed a subnormal glomerular filtration rate during the control period which decreased after starting the diet. No change was noted in the effective renal plasma flow. The tubular excretory mass was observed to decrease on the diet.

The change in blood pressure observed was not associated with a significant change in plasma volume or serum electrolytes. The addition of sodium chloride resulted in a rise in blood pressure. A persistent negative nitrogen balance was noted.

Metabolic Studies in Infants During Recovering from Vomiting. T. S. DANowskI and (by invitation) $L$. Greenman, J. H. Peters, R. Gow and F. Mateer, Pittsburgh, $\mathrm{Pa}$.

Six infants with pyloric stenosis, together with 1 other with vomiting and diarrhea of undiagnosed origin, have been studied during convalescence by the balance method. The majority had characteristic hypochloremia and alkalosis. Azotemia was present in one. At the start of the individual studies, but following therapy with solutions of saline and glucose, 4 had abnormally low concentrations of serum potassium. The initial serum sodium ranged between 123 and 152 milliequivalents per liter.

Oral intake was then completely withheld, without further vomiting, until the obstruction had been relieved. During this time the patients received parenteral glucose solutions, small amounts of saline, and, in all but one instance, practically no potassium. The potassium intake again became significant, however, upon resumption of oral fluids and food.

During recovery while on either parenteral or oral intake all subjects retained sodium and chloride. The chloride space, assumed at the start to be 15 per cent of the initial body weight for purposes of calculation expanded progressively until, in some instances, it exceeded by a considerable margin the volumes usually assigned to extracellular fluid. Hypokalemia appeared, or usually increased further if already present, in six patients. Low levels developed even though urinary losses were only a fraction of a milliequivalent per day. With potassium administration the urinary excretion increased, but did not equal the intake. This retention points to preexisting deficits and suggests renal conservation of potassium. The data do not identify the renal and extra-renal losses of potassium in the etiology of potassium deficits incurred prior to these studies. They do point, in spite of undue rises in the chloride space employed to partition external balances between cells and extracellular fluid, to a combination of dilution of extracellular fluid and transfer of potassium into cells as the mechanism of hypokalemia during therapy.

Antifibrillatory Actions of an Alpha-Fagarine Analog (N-Methyl-N-(3-4 dimethoxybenzyl) - B - (4, methoxyphenyl) - Ethylamine - HCL). JoSEPH R. DIPalma, (by invitation) John E. Schults, Richard A. Reiss and Joseph J. LAMbert, Brooklyn, N. Y.

The exact chemical structure of $\alpha$ Fagarine is unknown. A new compound, approaching its proposed formula and having similar biological effects and superior in respects to potency with low toxicity has been synthesized.

In cats the new compound is 2.3 times more active than quinidine and 1.3 times more active than $\alpha$ Fagarine in stopping electrically induced auricular fibrillation. The effects on blood pressure, pulse and EKG are similar to quinidine. Nevertheless, its acute i.v. toxicity for cats is less than either quinidine or $\alpha$ Fagarine.

When assayed in humans with normal hearts the Q-T segment is increased on the average by $0.06 \mathrm{sec}$. with a dose of $100 \mathrm{mgm}$. and by $0.09 \mathrm{sec}$. with a dose of 200 mgm. i.m. Similar doses of quinidine in the same patients increased the Q-T segment by 0.01 and $0.03 \mathrm{sec}$. respectively. This may be taken as good evidence that the drug is capable of increasing the refractory period of heart muscle and that it is more potent than quinidine in this respect.

In 5 patients with chronic auricular fibrillation the $f-f$ interval was increased on the average 28.2 per cent with a dose of $100 \mathrm{mgm}$. i.m. This slowing of the rate of auricular circus movement is much greater than could be anticipated from a similar dose of quinidine.

Clinical study of 10 patients, 7 with auricular fibrillation, 2 with auricular flutter and 1 with ventricular tachycardia permit the following conclusions. Two cases of auricular fibrillation and one with flutter were restored to a regular sinus rhythm. In one instance restoration occurred with a dose of only $50 \mathrm{mgm}$. i.m. A very serious disadvantage of the drug is that like $\alpha$ Fagarine it has a tendency to initiate ventricular ectopic foci. Because its chemical structure is known the possibility exists of developing a derivative of equal potency but lacking this undesirable feature.

\section{A Method for Estimating, preoperatively, the Function of Thyroid Tumors; Its Significance in the Diagnosis and Treatment. Brown M. Dobyns and Bengt Skanse (Introduced by Edward D. Churchill), Boston, Mass.}

An estimate of function of a discrete thyroid nodule contributes in a major way to the diagnosis and treatment of such a goiter. Many studies indicate that function of such nodules can be related to their avidity for radioiodine. An in vivo technic has been devised for detecting the degree of concentration of radioiodine in various parts of the thyroid. This technic permits an estimation of the relative function of discrete nodules as compared with the remaining thyroid.

The conventional Geiger-Muller counters are incapable of locating point sources within the thyroid because of the anatomical and physical relationships involved. 
A specially constructed directional counter has been found capable of locating and differentiating relative concentrations of radioiodine over small areas of the thyroid. In more than $\mathbf{5 0}$ patients the results of observations made with this technic were found to agree with radioautographs made from the same tissue removed at operation. Such differentiation in the function of thyroid nodules contributes significantly to the intelligent treatment of these goiters.

Hyperfunctioning adenomas of the thyroid are recognized as a source of clinical or masked hyperthyroidism which may produce cardiac damage. Their recognition and removal are often followed by remarkable improvement. This method of preoperative study has been of value in recognizing many such lesions.

By this method of locating the concentrated radioiodine, an incidental non-functioning nodule in the thyroid of Graves' disease may be distinguished from a hyperfunctioning adenoma producing hyperthyroidism. Since the management of these two problems may differ, the observations are important.

In our experience cancer of the thyroid collects less radioiodine than normal thyroid tissue. The finding of a nodule with a decreased or absent function eliminates the large group of hyperfunctioning nodules and thus increases our suspicion of malignancy.

Pulmonary Circulatory Dynamics in Mitral Stenosis and Left Heart Failure. J. W. Dow (by invitation), L. DeXter, and (by invitation) F. W. HAynes, J. L. WhitTenberger, and B. G. FerRIs, Boston, Mass.

Pressures in the pulmonary artery and pulmonary "capillaries" were measured with Hamilton and saline manometers. Blood flow through the lung was determined by the direct Fick principle. Pulmonary arteriolar resistance was determined by the Poiseuille equation.

In patients with mitral stenosis and left heart failure, pulmonary "capillary" pressure was elevated. In mild cases, the pulmonary artery pressure was likewise elevated, while the pulmonary artery-pulmonary "capillary" pressure gradient, blood flow, and pulmonary arteriolar resistances remained normal. In more advanced stages, the pulmonary "capillary" pressure at rest exceeded the osmotic pressure of plasma, the pulmonary artery pressure became extremely elevated, and the pulmonary arteriolar resistance rose to high levels due in part to a decreased cardiac output but mainly to a high pulmonary artery-pulmonary "capillary" pressure gradient.

It is concluded that in mitral stenosis and left heart failure (1) the pulmonary "capillary" pressure is elevated, sometimes exceeding the osmotic pressure of plasma, (2) the pulmonary artery pressure rises to a similar extent in early cases and in advanced cases the rise is out of all proportion to the rise in pulmonary "capillary" pressure due to constriction of the pulmonary arterioles thus producing an obstruction to blood flow proximal to the pulmonary capillary bed.

Effect of Treatment on Renal Functions and Electrolyte Excretion in Congestive Heart Failure. DAvD P.
Earle, Jr., (by invitation) SaUl J. Farber and John D. Alexander, and Ludwig W. Eichna, New York, N. Y.

Serial observations on renal functions, daily electrolyte excretions and cardiovascular status were made on 2 patients during various phases of congestive heart failure. They were maintained on regular hospital diet and moderate ward activity, and received no therapy other than digitalis products. Glomerular filtration rate $(\mathrm{GF})$, renal plasma flow (PF) and $\mathrm{TmPAH}$ were reduced while the filtration fraction (FF), venous pressure (VP) and weight were elevated in both during the congestive failure phase. As edema was lost there was fall in VP and FF and some improvement in GF and PF. These changes, however, were slight and the functions did not return to normal. Electrolyte excretions paralleled water balance, but at no time did electrolyte excretions fall to very low levels.

The acute effects of intravenous digoxin on the above functions were also studied. Rather prompt increases in water and sodium excretions were noted, even in the absence of significant change in GF, PF or FF. Water and electrolyte excretions appeared to be inversely related to the VP. In instances where VP fell but remained elevated well above normal, little or no increase in water or electrolyte excretion was observed. Similarly, marked diuresis was observed following intravenous injection of aminophyllin, without significant change in renal hemodynamics.

These data are compatible with the thought that impaired water and electrolyte excretion in congestive heart failure is not necessarily related primarily to the commonly observed changes in renal hemodynamics. It appears that increased renal venous pressure may play an important role in retention of water and sodium in congestive heart failure of man. Data bearing on the possibility that cardiac glycosides may act on renal tubular mechanisms of electrolyte reabsorption is also presented.

\section{Effect of Protein Starvation on the Clinical Course, Liver Function, and Liver Histochemistry of Patients wtih Active Liver Disease. Richard EckHardT, Norman Zamcheck, Richard Sidman and George Gabuzda (Introduced by Maxwell Finland), Boston, Mass.}

To clarify the role of protein in the treatment of human liver disease, 3 deeply jaundiced patients with active alcoholic cirrhosis (fatty) were provided with food adequate in calories but not sufficient in protein to maintain nitrogen equilibrium $(0.5,0.2$, and 0.0 grams per kilogram body weight daily for 24,8 , and 11 days, respectively). Although negative nitrogen balance persisted, each manifested clinical improvement, a decrease in liver size and tenderness, a rapid fall in serum bilirubin and in urine bile, and a progressive decrease in urine urobilinogen (measured in 2 patients). Nitrogen balance became positive when 1.0 to 1.4 grams of protein per kilogram daily were given. Improvement continued, but appeared less marked because of the extent of previous change. The serum albumin concentration and thymol turbidity and 
flocculation tests, although frequently abnormal, showed no consistent pattern of change.

Histochemical studies were made of liver tissue obtained by biopsy from the last 2 patients before and during the low protein period and during the increased protein feeding period. Five "protein starvation" biopsies showed an initial low cellular protein content which further diminished, moderate to marked fat which remained unaltered, and shrunken cells. Necrosis was not observed. The three "protein repletion" biopsies showed a marked increase in protein content, a progressive decrease in fat, and a return of cell size and appearance toward normal. Liver glycogen progressively increased during both periods of study, while fibrosis (minimal) remained unchanged.

It is concluded that, under the conditions of this study, improvement in clinical status and in liver function occurred in spite of negative nitrogen balance occasioned by diets grossly deficient in or devoid of protein. Liver histology, however, failed to reflect this improvement, and morphologic improvement appeared only with the provision of adequate exogenous protein and the maintenance of positive nitrogen balance.

Studies on the Pathogenesis of Nephrotic Edema. HowARD A. EDER and Francis P. CHINARd (by invitation), Henry D. Lauson, and (by invitation) Roger L. Greif, Alma Hiller, George C. Cotzias and Donald D. VAN Slyke, New York, N. Y.

The availability of concentrated human albumin for intravenous administration has made possible extensive studies of the sequence of events occurring during the development of nephrotic edema. The initial event is proteinuria. The renal mechanism involved has been examined in nephrotic patients by measurements of the albumin clearance under conditions of changing plasma concentrations following infusion of albumin. It has been found that the excretion is proportional to the plasma concentration, and that the albumin/creatinine clearance ratio is constant over a wide range of concentrations, unless the blood volume increases, in which case the ratio also increases. Our present concept is that in nephrosis albumin is filtered through the glomerular membrane in abnormally large amounts, and that almost all of the filtered albumin is excreted, tubular reabsorption being relatively slight. The continued excretion of albumin results in hypoalbuminemia, and, in accordance with the Starling hypothesis, the plasma volume decreases absolutely as well as relative to the extracellular fluid volume. The diminished plasma volume is believed to be responsible for the decreased renal excretion of water and salt which is the immediate cause of edema. This belief is based on the observation that the excretion of these substances rises sharply when the plasma volume is expanded following the administration of albumin. The fact that the rate of glomerular filtration is increased at the same time suggests that the retention of salt and water during accumulation of edema may be attributed at least in part to a decrease in the rate of glomerular filtration, relative to the intrinsic (i.e., non-hormonal) tubular reabsorptive activ- ity with respect to water and salt. In addition, there is some evidence to suggest that increased secretion of saltactive adrenal hormones and antidiuretic hormone may play a role in the retention of salt and water.

The Mechanism of Anemia and Its Inflence on Renal Function in Chronic Uremia. Charles P. Emerson, JR., and BeLton A. Burrows (Introduced by Chester S. Keefer), Boston, Mass.

The assumption that anemia in uremic patients is due to inadequate blood production rather than to excessive blood destruction has been tested in four cases of chronic nephritis with marked anemia and azotemia. Hematologic and metabolic studies were performed before and after successive transfusions of marked red cells which in each instance could be distinguished by means of selective agglutination technics from those of the recipient. The relative rates of erythropoiesis and hemolysis were estimated from the results of serial hematologic examinations, including separate measurements of the concentration and total volume of circulating donor and recipient red cells, the degree of reticulocytosis and of bilirubinemia. Parallel determinations were made of the concentrations of non-protein nitrogen and electrolyte constituents of the blood, the total volume of extracellular fluid, and the volume and composition of the urine, as a basis for evaluating the influence of anemia and the effect of corrective blood replacement on renal function.

The data obtained indicated that anemia in these cases was mainly attributable to a depression of erythropoiesis, but also suggested that a contributory factor in its pathogenesis was the presence of excessive hemolytic activity, evidenced by the unusual rapidity with which donor red cells were destroyed following transfusion, their elimination from the blood of the recipient occurring at 1.5 to 3 times the normally expected rate. With respect to the clinical and metabolic effects of massive transfusions it was impossible, in this series of patients, to demonstrate any correlation between the degree of renal decompensation and the grade of coexisting anemia. Complete correction of the latter by means of artificial replacement therapy failed in all instances to lessen or to increase the severity of the uremic manifestations or to influence the progress of the underlying renal disease.

The Insulin Glucose Tolerance Test-An Improved Procedure for the Detection of Hypoglycemia Unresponsiveness in Pituitary and Adrenal Insufficiency. FRANK L. Enger and (by invitation) James L. Scotr, Durham, N. C.

The usefulness of the insulin tolerance test for the diagnosis of conditions characterized by hypoglycemia unresponsiveness is limited by the danger of serious hypoglycemic reactions in those patients in whom the test should be most valuable. Smaller doses of insulin often yield equivocal results requiring repetition of the test. To overcome these objections advantage has been taken of the fact that hypoadrenal and hypopituitary patients not only exhibit hypoglycemia unresponsiveness after insulin but also a de- 
layed response to oral glucose during hypoglycemia. By giving $0.8 \mathrm{gm}$. of glucose per kilogram orally to the fasted subject 30 minutes after 0.1 unit insulin per kilogram intravenously, hypoglycemic manifestations are aborted but hypoglycemia unresponsiveness is still apparent. Blood sugars are determined before insulin and at $30,60,90,120$, and 180 minutes. The results are expressed as per cent of the fasting blood sugar. In 9 normal individuals and $9 \mathrm{pa}$ tients with non-endocrine diseases the mean maximal rises in blood sugar after glucose were 149 and 128 per cent of the initial blood sugar at 90 minutes with a return to the fasting level at 180 minutes. In 7 observations on Addison's disease and 6 on panhypopituitarism the mean maximal rises were only 82 and 84 per cent of the initial blood sugar respectively and occurred at 180 minutes. None of the latter patients achieved a blood sugar after insulin and glucose as high as the lowest peak in the two control groups. As a group, 6 patients with anorexia nervosa had a mean response not significantly different from the controls. However, one patient fell clearly into the hyporesponsive range and one was borderline. Three patients with primary myxedema showed normal curves. Neither pretreatment with lipoadrenal cortex, intravenous cortical extract $(50 \mathrm{ml}$.) or epinephrine before and after insulin restored the response of the hyporesponsive patients to normal.

\section{Skin Reactions to Toxoplasma Antigen in People without Known History of Infection. HARRY A. FELDMAN and Albert B. SABIN, Cincinnati, Ohio.}

One hundred and forty-two residents of the Cincinnati area ranging in age from 3 months to 81 years were surveyed for their reaction to the intracutaneous injection of toxoplasma antigen prepared from infected chorioallantoic membranes of chicken eggs. The positive reactions, consisting of red, indurated, swollen, tender and itching lesions ranging in size from $60 \times 50 \mathrm{~mm}$. to $10 \times 10 \mathrm{~mm}$. (the latter being relatively rare), reached their maximum in 30 to 48 hours. The incidence of positive reactions among at least 20 individuals in each age group listed was as follows : $0-4$ years -0 ; 5-9 years $-5 \%$; $10-19$ years $-14 \%$; 20-29 years-20\%; 30-39 years-50\%; 40-49 years$65 \%$; $50-81$ years-65\%. A positive skin test was invariably associated with demonstrable toxoplasma antibody in the serum. Toxoplasma antibody in varying titer was also found in the serum of 7 of 18 persons (39\%) with negative skin tests. Further evidence in favor of the high incidence of toxoplasma antibody among the "normal" adult population was obtained when a 15-20 fold concentrated, commercial preparation of gamma globulin prepared from the plasma of more than 20,000 American adults was found to contain toxoplasma antibody in a titer of $1: 256$ as determined by the dye test. No agent having an antigen in common with toxoplasma and capable of giving rise to antibodies for this protozoon parasite has been found thus far. Therefore, one must consider the possibility that inapparent infection with toxoplasma may be very widespread, although it is rather curious that the incidence of antibodies (and presumably infection) should rise so sharply after the 20 th year of life. The toxoplasma skin test is not useful for diagnosis because a strongly positive test bears no relationship to titer of antibody and a negative test is encountered too often in the presence of antibody.

Studies on the Isolation and Identification of Beta-Hemolytic Streptococci from Throat Swabs. A. E. FeLler, (by invitation) GeOrge F. BAdGer, JohN H. Dingle, (by invitation) Richard G. Hodges, and Charles H. Rammelkamp, Cleveland, Ohio.

Investigations of the role of $\beta$-hemolytic streptococci in respiratory disease require (1) sensitive methods for detecting the organisms on throat swabs, especially when they are present in small numbers, and (2) reliable methods for characterizing the organisms so isolated. For the latter purpose, classification according to the serological groups of Lancefield is essential.

The purposes of the present study were (1) to compare the sensitivity of the conventional streak plate method with the technique devised by Pike employing selective media for the detection of $\beta$-hemolytic streptococci on throat swabs, (2) to devise a simple method for predicting that a certain organism can be grouped serologically, short of making an extract of each organism and testing it against group specific sera, and (3) to determine the reliability of the rather simple enzyme technique devised by Maxted for preparing extracts of the organisms for serological identification.

Comparison of the streak plate method with the Pike technique showed that 96 per cent of 327 consecutive isolations of groupable streptococci were accomplished by the Pike method; in only $4 \%$ of the swabs was the streak plate positive when the Pike plate was negative. After evaluating many procedures it was found that $\beta$-hemolysis of sheep blood agar (surface or deep colonies) is a simple and reliable criterion for determining that a streptococcus can be grouped serologically (Lancefield). Organisms failing to produce $\beta$-hemolysis on sheep blood agar have thus far not been groupable. Comparison of the enzyme technique of Maxted with the conventional formamide method of preparing extracts of streptococci for serological grouping has demonstrated essentially complete agreement when employing streptococci producing $\beta$-hemolysis on sheep blood agar. The Maxted technique is extremely simple and the extracts react with specfic sera in 15 to 30 minutes.

Iron Metabolism in Hemochromatosis. C. A. FINCH (Introduced by James P. O'Hare), Boston, Mass.

Observations have been made relating to the diagnosis, pathogenesis, and treatment of hemochromatosis.

Iron excess may be recognized by sternal puncture, by the per cent utilization of radioiron for hemoglobin production, and by determining the per cent saturation of the iron binding protein of the serum. The latter has proved particularly useful in the recognition of hemochromatosis.

Since little iron is excreted in man, hemochromatosis must be associated with increased iron absorption. In animals it has been possible by dietary means to produce excessive iron absorption, indicating that the normal mu- 
cosal regulation of iron absorption is not effective in certain circumstances. It is also suggested by similarity in iron distribution in these experimental animals, in patients with multiple transfusions and in idiopathic hemochromatosis, that the iron in all instances represents storage iron. Accordingly several patients have been repeatedly phlebotomized. In contrast to normal subjects who rapidly exhaust their iron reserves and develop anemia, patients with hemochromatosis are able to give up large amounts of iron without evidence of iron deficiency.

These studies provide methods of identifying enlarged stores of iron at an early stage in the clinical course of hemochromatosis and by phlebotomy a means of removing the excessive iron found in this disease.

Sweat Sodium Concentration in Thyrotoxicosis. FrancIs W. Fitzhugh and Arthur J. Moseley (by invitation), and Arthur J. Merrill, Atlanta, Ga.

Conn has demonstrated an inverse relationship between the sweat sodium concentration and the activity of the sodium-retaining factor of the adrenal cortex. In the course of other studies, we found a low sweat sodium concentration in 10 of 11 patients with thyrotoxicosis. Our average for 26 determinations in 18 normal subjects was $49.1 \mathrm{mEq}$./1. with a range of 15 to $92 \mathrm{mEq}$./1., all but 4 being between 24 and 70 . The average for 10 of 11 patients with thyrotoxicosis was $14.2 \mathrm{mEq}$./1. with a range of 2.4 to 26.6. The remaining patient had a sweat sodium concentration of $46.2 \mathrm{mEq} . / 1$. The BMR's of these patients ranged from +42 to +80 .

Five normal individuals were placed on thyroid extract, 2 grams daily. Three of these had a drop in sweat sodium concentration from 51 to 31,61 to 32.5 , and 92 to 22 on the 7th, 7th, and 4th days respectively. One subject had a low control level of $19 \mathrm{mEq} . / 1$. and his sodium concentration rose to 25 on the 7th day. The fifth individual showed an increase from 32 to $63 \mathrm{mEq}$./1. on the 10th day. Two of those who had a fall in sweat sodium concentration were restudied with daily sweat sodium determinations. One of these had a marked diminution on the 9th day from 38 to 19.5 followed by a rebound to 42 on the 11 th day. The other showed no significant change. Reciprocal changes occurred in the sweat potassium concentration. No change was noted in the number of circulating eosinophils or in the BMR.

The determination has been of assistance in diagnosis in two patients with thyrotoxicosis whose BMR's were unsatisfactory and in whom the diagnosis was uncertain.

In summary, a new method is offered for the diagnosis of thyrotoxicosis which shows little overlapping with the normal.

Metabolic Studies on Cushing's Syndrome. P. H. ForSHAM and E. FLINK (by invitation), K. EMERSON, Jr. and G. W. Thorn, Boston, Mass.

Eosinopenia (less than 50 cells per cu. mm.) appears to be characteristic of Cushing's syndrome and an elevated urinary uric acid: creatinine ratio (greater than 0.8 ) frequently occurs. Such changes have been reproduced in normal subjects given adrenocorticotrophic hormone and in patients with Addison's disease on Compounds $E$ and F. Following successful treatment, these values return to normal and their change constitutes a helpful indicator of the effectiveness of therapy.

The refractoriness of certain patients with Cushing's syndrome to the salt retaining action of desoxycorticosterone (Soffer) has been confirmed. In a study on the metabolic effects of Compound $E$ in patients with Addison's disease it was found that $100 \mathrm{mg}$. of Compound $\mathrm{E}$ daily led to slight sodium and chloride retention. In contrast, such patients when treated with desoxycorticosterone and showing marked sodium retention exhibited a paradoxical increase in sodium and chloride excretion when given additional Compound E. On the basis of these observations on the competitive renal action of desoxycorticosterone (a powerful salt retainer) and Compound $E$ (a weak salt retainer), it is suggested that the desoxycorticosterone acetate fastness of certain patients with Cushing's syndrome may be due to the blocking action on the renal tubule of an excess of adrenal steroids of the 11,17-oxytype.

Electrolyte Abnormalities in Chronic Congestive Heart Failure; Effects of Administration of Potassium and Sodium Salts. Chardes L. Fox, JR., Charles K. Friedburg and Abraham G. White (Introduced by A. R. Dochez), New York, N. Y.

Plasma, edema fluid and urine electrolytes were studied during congestive failure. Plasma sodium was significantly below normal in twenty-seven of thirty patients. Plasma chlorides and potassiums ranged from below to above normal; chlorides, however, were relatively increased. Urinary sodium was low whereas potassium was high. Repeat measurements after recovery in five patients showed that the low sodiums had increased, whereas chloride and potassium shifted toward normal.

During mercurial diuresis sodium chloride excretion increased but sodium in urine was usually hypotonic; chlorides however equalled or exceeded sodium. Potassium excretion increased fivefold.

The data indicated that failure is frequently associated with hypotonic extracellular fluid excessive in chloride and that during recovery, relatively more water, chloride and potassium are excreted than sodium. The possibility that some of the water excreted was of intracellular origin is also supported by the rise in the non-colloidal extracellular osmotic pressure which might draw water from cells.

Accordingly, sodium lactate was given to raise extracellular sodium and bicarbonate concentrations, and potassium acetate to raise intracellular potassium and bicarbonate. Up to $400 \mathrm{mEq}$. of each were administered orally for periods up to thirty days to six patients in failure. Mercurial diuretics previously required were omitted. Loss or no gain in weight occurred despite positive balances of sodium and potassium. Sodium excretion increased markedly although subnormal plasma sodium initially decreased further. Shift of water from intra- to extracellular compartments is thereby suggested.

Individual administration of the chloride and acetate of $\mathrm{Na}$ or $\mathrm{K}$ increased weight two to six kilograms therefore 
mercurials were required after each single salt period. Simultaneous administration of sodium chloride and potassium acetate caused minimal weight gain despite positive $\mathrm{Na}, \mathrm{Cl}$, and $\mathrm{K}$ balances.

Extracellular hypotonicity may be associated with intracellular hypotonicity and depletion, hence separate readjustment of either compartment augments edema. Intracellular abnormalities may also be important in failure.

Quantitative Studies on Pulmonary Absorption of Aerosols Made with Solutions of Sodium p-Amino Hippurate. William Franklin and Clarence Denton (by invitation), and FraNCIS C. Lowell, Boston, Mass.

In the course of laboratory studies on the effect of aerosolized drugs and allergenic extracts in pulmonary disease, the need arose for a method of determining, with some degree of accuracy, the amount of material retained by the subject. The use of penicillin (blood levels) or radioactive materials (tissue concentrations) was considered and discarded as poorly adapted to the problem. Promising results have been obtained with aerosolized solutions of sodium p-amino hippurate. This compound is well tolerated and is rapidly absorbed and quantitatively excreted in the urine. The procedure followed was (1) to expose the subject to a measured volume of a standard aerosol of PAH, (2) to collect material not retained by the subject, and (3) to collect the urine over a six hour period, with determination of the amount of $\mathrm{PAH}$ after hydrolysis with acid.

The aerosol, produced by nebulizers receiving oxygen delivered at an accurately measured rate, was passed over concentrated sulfuric acid in order to increase stability by partial drying into a spirometer (which provided the flexibility required by the intermittent removal of the areosol during the subject's respiration) and through a flutter valve to the subject. Expired aerosol passed through a second flutter valve and was collected in a highly efficient impinger. Measurements were made of the PAH delivered, the $\mathrm{PAH}$ in the collecting impinger, the valve assembly, and the urine.

Repeated tests have shown that agreement within 10 per cent was obtainable between (1) the amount delivered and (2) the sum of that found in the urine, the expired air and valve assembly. The following results were obtained in one test with subject inhaling aerosol for ten minutes: amount of PAH delivered-96 mgms.; amount in expired air and valve assembly- 54 mgms.; amount in urine -42 mgms.

Studies with a New Method for Quantitative Measurcment of $I^{121}$ Content of the Thyroid Gland in Man. A. STONE Freedberg, Alvin Ureles and Marvin Van Dilla (Introduced by H. L. Blumgart), Boston, Mass.

Direct measurement of uptake of radioactive iodine by the thyroid has been attempted by two techniques of external counting: (1) a Geiger-Mueller counter is placed against the neck over the thyroid gland; (2) the tube is at varying distances from the neck. In both instances, the variation in thyroid size and location (depth geometry and absorption) have not permitted quantitative measurement of $I^{181}$ content.

With 4-6 platinum cathode Geiger-Mueller counters connected in parallel and arranged in a circle $36-120 \mathrm{~cm}$. in diameter, a central source of radiation was moved about within a sphere $8-32 \mathrm{~cm}$. diameter concentric with the circle of counters; the maximum variation in counting rate was $\mathbf{5}$ per cent. When the source was surrounded by muscle, bone, water and saline in quantities approximating the human neck there was no significant change in counting rate. With a constant amount of radioactivity in volumes varying from 5-350 cc. in different shaped containers no significant change in counting rate was observed. Identical amounts of $I^{132}$ in 5 cc. and in 2,000 cc. of water placed in gallon jugs showed a decrease of 5 per cent in the counting rate in the latter instance.

Preliminary studies in patients with varying thyroid gland size are consistent with mock up experiments. With a 4 tube circle of $104 \mathrm{~cm}$. diameter, a central "sphere" is present approximately $20 \mathrm{~cm}$. in diameter within which movement of the head and neck gave no significant change in counting rate. Thyroid uptake and urinary excretion has been measured by this method in euthyroid subjects and in patients with various thyroid disorders and will be reported.

It is concluded that quantitative measurement of $I^{131}$ content of the thyroid gland in man is obtained by the above described method independent of the size and location of the gland.

Evaluation of the Dye Method for Estimating Blood Volume in Man. EDWARd D. Freis, Joseph R. Stanton and Charles P. Emerson (Introduced by William L. Fleming), Boston, Mass.

The higher estimation of blood volume using T-1824 as contrasted with labeled red cells has cast doubt on the validity of dye methods for determining blood volume. In man the mixing time of $\mathrm{T}-1824$ was relatively complete within the first three minutes following which there was a uniform and therefore detectable disappearance of circulating dye which could be corrected by extrapolation. Repeated injections of $\mathrm{T}-1824$ at short intervals indicated that there was no undetected dye loss during the initial mixing period. Simultaneous determinations of arterial and hepatic venous concentrations of dye failed to demonstrate significant escape of T-1824 in the liver.

Measurement of the relative velocities of dyed plasma and identified red cells during a single circulation through a peripheral vascular bed revealed that in the capillaries the mean velocity of the cells was greater than that of plasma. The capillary hematocrit, therefore, must be lower than the large vessel hematocrit. These observations support the validity of the dye method for measuring plasma volume and indicate that the high values obtained for total blood volume with T-1824 are not due to undetected escape of dye from the circulation but to the discrepancy in the proportion of red cells and plasma in large versus small vessels. 
A Quantitative Study of the Rate of Renal Excretion of Digitoxin. MEYER FRIEDMAN and (by invitation) Rene Bine, Jr. and Sanford O. Byers, San Francisco, Calif.

Hitherto, it has not been possible to determine with quantitative methods the role of the kidney in the excretion of digitalis glycosides. However, by means of the embryonic duck heart method, as little as one microgram of digitoxin could be detected in the 24 hour urinary output of the rat. This technique therefore enabled us to study the rate of renal excretion of digitoxin in this animal after its parenteral administration.

A series of 10 rats were given by intramuscular injection $0.1 \mathrm{mcg}$. of digitoxin per gram of body weight (or approximately $15 \mathrm{mcg}$. per rat) and the urine was collected daily for three days. Each 24 hour urine sample was analyzed for its digitoxin content. No digitoxin could be detected in the initial 24 hour urine collection of any rat, indicating that less than one microgram of digitoxin had been excreted during this period. Likewise no digitoxin was found in the 48 or 72 hour urine sample of any rat.

A series of 12 rats therefore were injected with $1.0 \mathrm{mcg}$. of digitoxin per gram of body weight (approximately 225 mcg. per rat). It was found that there was an average excretion of $6.0 \mathrm{mcg}$. of digitoxin $(2.5$ per cent of amount injected) during the first 24 hours following injection. No digitoxin, however, was detected in any of the urine samples collected during the 48 or 72 hours following injection.

The above results indicate that the renal excretion of digitoxin in the rat is either nil or relatively insignificant even after the administration of relatively huge amounts of the drug.

Effect of Lipotropic Agents, Testosterone Propionate, and Dietary Protein upon Nitrogen Balance in Patients with Liver Disease. GroRge J. GABUzDA and RICHARD D. EckhardT (by invitation) and Charles S. Davidson, Boston, Mass.

Four patients with Laënnec's cirrhosis and one with infectious hepatitis were maintained on basal diets providing $25,50,75,100$ and 100 grams of protein daily. Initial control nitrogen balances averaged $-4.2,+1.6,+3.3,+5.4$, and +6.8 grams daily, respectively. The effect of supplements of choline and methionine, of testosterone, and of dietary protein upon these balances was determined.

The oral administration of 4.5 to 9.0 grams each of choline chloride and dl-methionine simultaneously daily for 6 to 9 days to these 5 patients did not have a nitrogen sparing effect above that which could be accounted for by retention of part of the nitrogen contained in these agents.

Subsequent to control periods following the administration of choline and methionine, $25 \mathrm{mgm}$. of testosterone propionate was administered intramuscularly daily for 6 days to the 3 patients with intakes of 50,100 , and 100 grams of protein daily. Their ability to respond to a protein anabolic stimulus was indicated by increased nitrogen retentions of 1.2, 2.6, and 1.4 grams daily.
After the reestablishment of nitrogen balances comparable to initial control periods, increases in dietary protein of 50 grams daily in the 3 patients on basal intakes of 50,75 , and 100 grams of protein were accompanied by increases in daily nitrogen retention averaging $2.6,3.8$, and 7.1 grams, respectively. The patient in negative nitrogen balance while receiving a 25 gram protein basal diet promptly established nitrogen equilibrium when the protein intake was increased to 50 grams, although previously choline and methonine had failed to spare nitrogen.

Although testosterone propionate had a protein anabolic effect in these patients with liver disease, it is concluded that positive nitrogen balance was most effectively achieved by adequate dietary protein. No appreciable nitrogen sparing action could be attributed to choline and methionine in the quantities used.

The Effect of Anoxic Anoxia on the Human Kidney. Morton Galdston, Eugene Y. Berger, and Seymour A. Horowitz (Introduced by J. Murray Steele), New York, N. Y.

Several factors, among them anoxia, may play a part in retention of salt and water in heart failure. The following experiments were designed to test the effect of renal anoxia per se on these phenomena. In 7 subjects with normal cardiovascular, respiratory systems, renal function during inhalation of 14 per cent oxygen (arterial oxygen tension of approximately $50 \mathrm{~mm} . \mathrm{Hg}$ ), a degree of anoxia which results in barely perceptible changes in circulatory and respiratory dynamics, was compared with renal function during the preceding and subsequent periods breathing room air. Similar studies were conducted in 2 emphysematous subjects with arterial blood oxygen tensions of approximately $50 \mathrm{~mm}$. $\mathrm{Hg}$ while breathing room air and again, while breathing 100 per cent oxygen. Periods breathing room air were considered as the anoxic periods. Observations on respiration and circulation were also made.

Renal plasma flow remained the same or tended to increase during anoxia in all normal subjects. In the emphysematous subjects it decreased during inhalation of 100 per cent oxygen. Change in filtration rate during anoxia was not consistent in direction or degree. The most pronounced effect of anoxia on renal function was an invariable and frequently marked increase in the rate of excretion of sodium, chloride, and water. The alterations in respiration and circulation which occurred during inhalation of 14 per cent and 100 per cent oxygen do not seem to account for the changes in renal plasma flow. The conclusion is drawn that anoxic anoxia alone does not appear to result in reduction of renal plasma flow or retention of salt and water. Other electrolyte changes studied will be presented.

Transfer to Normal Red Cells of an Agglutinin Demonstrable in the Acidified Sera of Patients with Acquired Hemolytic Jaundice. FranK H. GARDNER (Introduced by Thomas Hale Ham), Boston, Mass.

Studies of defibrinated blood samples from 15 patients with acquired hemolytic jaundice have shown agglutina- 
bility of the red cells with anti-human immune rabbit serum (direct Coombs test). In certain of these patients, although the adult red cells were strongly agglutinated in the Coombs test, the reticulocytes were not. Following transfusion, normal red cells became agglutinable in the Coombs test and were thereafter rapidly destroyed (Ashby technique). This evidence suggested that the patient's, as well as normal red cells, had adsorbed a globulin substance in vivo which was detected by the Coombs test. Normal red cells, incubated with splenic pulp from these, but not from patients with congenital hemolytic jaundice acquired agglutinability in the Coombs test.

In 13 of the 15 patients, lowering of the $\mathrm{pH}$ of the serum to the range of 6.5 to 6.7 produced, on incubation, agglutination of the patient's red cells and of normal homologous red cells. When the $\mathrm{pH}$ of these mixtures was subsequently raised to 8 , the agglutination was abolished, but, in all instances the normal red cells had acquired agglutinability in the Coombs test. However, in only 3 instances did normal red cells acquire agglutinabiilty in the Coombs test when incubated in the patient's serum at $\mathrm{pH} 8$. The material in the patient's serum, presumably an antibody globulin, could be adsorbed upon normal red cells at $\mathrm{pH}$ 6.5 and subsequently eluted at $\mathrm{pH} 8$ with normal serum.

It is suggested that in such instances of acquired hemolytic jaundice, exposure of the patient's red cells to a lowered $\mathrm{pH}$, and also to an increased concentration of antibody globulin in such organs as the spleen causes them to become agglutinable and consequently subject to sequestration and destruction.

\section{Effect of Potassium Deficiency on Carbohydrate Metabol-} ism. LyTT I. Gardner (by invitation), NathaN B. Talbot and (by invitation) Charles D. CoOK, Helen Berman and Concepcion Uribe R., Boston, Mass.

In uncontrolled diabetes and in some cases of Cushing's syndrome there occurs intracellular K deficit. Since both conditions exhibit abnormal carbohydrate metabolism, it was thought of interest to examine the effect of $\mathrm{K}$ deficiency on the metabolism of glucose and glycogen in the intact animal. A diet containing 0.003 per cent $\mathrm{K}$ and 0.7 per cent $\mathrm{Na}$ caused severe $\mathrm{K}$ deficiency in 160-175 gm. rats, with wasting and death between 60-120 days on the diet. $\mathrm{K}$ added to the diet produced excellent growth. The $\mathrm{K}$ deficient rats were divided into two groups: "A," sacrificed after 40-60 days on the diet and "B," after 90-120 days. Rats were fasted overnight, given glucose p.o. $1 \mathrm{gm}$. $/ 100 \mathrm{gm}$. body wt. and killed 4 hrs. later. Blood sugar was determined as $\mathrm{mg}$. per cent. Muscle and liver were analyzed for glycogen as $\mathrm{gm} . / 100 \mathrm{gm}$. wet tissue and for cations as $\mathrm{mEq} . / 100 \mathrm{gm}$. dry, blood-free, fat-free tissue. Group A and B muscle $\mathrm{K}$ was 72 per cent and $\mathrm{Na} 200$ per cent of control values, with lowest $K$ values and highest $\mathrm{Na}$ in Group B. Muscle $\mathrm{Mg}$ and $\mathrm{Ca}$ content was increased in Groups A and B. Liver $K$ was normal in Group A but reduced in some rats of Group $\mathrm{B}$. Liver $\mathrm{Na}, \mathrm{Mg}$ and $\mathrm{Ca}$ were increased in both groups. Group $A$ had normal blood sugar values $\left(120^{*} \pm 23.2\right)$ and normal glucose

\footnotetext{
* Mean \pm standard deviation.
}

tolerance tests, but liver glycogen $(6.56 \pm 0.98)$ and muscle glycogen $(1.53 \pm 0.93)$ were more than double control values (liver $2.47 \pm 0.34$, muscle $0.36 \pm 0.22)$. Group B had very high blood sugar values ( $555 \pm 221$ ), a high glucose tolerance curve, and low values for liver glycogen $(0.00-0.53)$ and muscle glycogen $(0.00-0.23)$. All $\mathrm{K}$ deficient rats showed increased adrenal wt./100 gm. body wt. Eosinophils/cu. mm. blood were low in Group A and almost absent in Group B. These data, together with increased glycogen levels of Group A, suggest chronic alarm reaction with increased production of "sugar-fat-nitrogen" adrenal cortical hormone. The diabetic response of Group $B$ would indicate that modification of the intracellular milieu had passed a critical threshold beyond which glycogenesis did not occur.

Tryptophan and Niacin Metabolism in Pellagra. GRACE A. Goldsmith and (by invitation) Herbert P. SARETt, New Orleans, La.

Three patients with pellagra were maintained on corn or wheat diets low in protein and niacin. Urinary excretion of niacin and tryptophan compounds was determined before therapy and following administration of 5 gm. of L-tryptophan daily. When DL-tryptophan is given to normal subjects there is an increase in the excretion of $\mathrm{N}^{\prime}$-methylnicotinamide $\left(\mathrm{N}^{\prime}-\mathrm{Me}\right)$, tryptophan, a niacin compound released by strong acid hydrolysis, and an ether extractable tryptophan-like compound. The last two metabolites, not normally present in urine in appreciable quantities, were excreted prior to therapy by one pellagrin who was starved, dehydrated and had azotemia. The other two subjects with pellagra, one with mild deficiency, the other with severe weight loss, dehydration and amebiasis, showed normal excretion of these compounds. $\mathrm{N}^{\prime}-\mathrm{Me}$ excretion by the latter two patients was low, whereas that of the azotemic subject was abnormally high. The excretion of $\mathrm{N}^{\prime}-\mathrm{Me}$, following tryptophan, increased gradually in all three subjects. In the two severely ill patients, administration of tryptophan was followed by a greater increase in the excretion of the acid-hydrolyzable niacin compound than was obtained with $10 \mathrm{gm}$. of DL-tryptophan in normal subjects. A patient without pellagra who had chronic nephritis and azotemia likewise excreted large amounts of this compound after $10 \mathrm{gm}$. of the racemic mixture.

Total tryptophan excretion was abnormally high following therapy in the subject with amebiasis. In the pellagrins, as in normal subjects, excretion of the ether-extractable tryptophan compound was less after L- than after DL-tryptophan.

Clinical improvement followed tryptophan administration in each patient but changes were less rapid than would have been anticipated with niacinamide. In pellagra, temporary derangements of tryptophan-niacin metabolism may occur but the ability to convert tryptophan to niacin is retained. Changes in renal function may partially account for excretary findings observed in pellagra.

Concentration of Iiodine in the Human Stomach and Other Tissues as Determined with Radioactive Iodine. RICH- 
ard E. Goldsmith and Charlis D. Strvens (Introduced by Leon Schiff), Cincinnati, Ohio.

The distribution of iodine, injected as iodide labeled with radioactive iodine, was measured in the tissues of 14 patients (with various ailments) coming to autopsy and 6 patients undergoing partial gastrectomy for gastric cancer or peptic ulcer to learn: (1) if human stomach tissue concentrates iodine above serum values; (2) if normal stomach tissue acts differently than carcinomatous stomach tissue in this respect; and (3) if the distribution of injected iodine among the tissues is affected by variations in the quantity of iodine given and variations in the period elapsing between iodine administration and death. Seven of these patients received approximately 200 milligrams of inert iodide, six received approximately 2 micrograms of inert iodide, and seven received only radioactive iodine. In each instance the administered iodine was labeled with 0.1 to 0.5 millicuries of radioactive iodine.

(1) The concentration of injected iodine in the stomach tissue was always above that of the serum and in one instance was greater than that in the thyroid gland. All other tissues examined showed much lower iodine concentrations. (2) No uniform differences were found between the concentrations of injected iodine in normal and in carcinomatous stomachs. (3) The proportion of the administered iodine taken up by the thyroid varied directly with the amount of iodine administered. All of the ratios of the tissue iodine concentration to serum iodine concentration increased directly with the time interval elapsing between administration of iodine and death.

\section{A Vectoral Study of Functional T-Wave Changes in the}

Electrocardiogram. RoBERT P. GRANT and E. HARVEY

Estes (Introduced by James V. Warren), Atlanta, Ga.

Using normal subjects, functional $T$ wave changes have been produced by the following methods: (1) increased intrathoracic pressure, (2) head-up tilting, and (3) digitalis administration. Mean spatial $Q R S$ and $T$ vectors and ventricular gradients were calculated by the use of a tetrahedral reference figure.

Under these circumstances the mean spatial $T$ vector is first shortened, then rotated away from the QRS vector, then lengthened in a direction away from the $Q R S$ vector. The ventricular gradient is shortened with comparatively little change in direction, which means that in the three procedures the forces altering the $T$ wave develop similarly at all regions of the ventricular myocardium.

Additional studies have shown that the effects of tilting and increased intrathoracic pressure on the $T$ vector are reversed during rapid intravenous saline infusions. This suggests that these effects are associated with diminished cardiac filling. Digitalis effects are not reversed by this procedure. Furthermore, the reduction in the gradient during digitalis administration takes place throughout the ST-T cycle, while in the other two procedures it is evident principally in the $T$ portion of the ST-T cycle. The meaning of these differences in terms of heart function will be discussed. From a practical point of view, the studies indicate that by using vectoral methods an abnor- mally directed $T$ vector can be identified even in the presence of digitalis effects.

The In Vivo and In Vitro Inhibition of Lysozyme by Aerosol (Di octyl sodium sulfo succinate). SEYMOUR J. Gray, Robert W. Reifenstein and Howard M. SPIRo (Introduced by Clifford L. Derick), Boston, Mass.

The fecal lysozyme activity of patients with ulcerative colitis was determined by the viscosimetric method of Karl Meyer; the lysozyme content varied from 88 to 462 units per gram of stool and decreased to 12 units per. gram or less in the healed or inactive phase of ulcerative colitis. The 24 hour fecal lysozyme output was from 49,940 to 111,000 units. Patients with other types of intestinal diseases and diarrhea, excreted 3 to 15 units per gram and 1,000 to 5,000 units in 24 hours depending upon the severity of the diarrhea. Normal individuals excreted less than 3 units per gram and 80 to 124 units in 24 hours.

In vitro studies indicate that Aerosol OT (Di octyl sodium sulfo succinate) is an effective inhibitor of lysozyme. One cubic centimeter of a 400 milligram per cent solution completely inhibited an equal volume of a diluted extract of ulcerative colitis stool containing 1.5 units of lysozyme. One cubic centimeter of a 40 milligram percent solution of Aerosol produced 74 per cent inhibition of the enzyme.

Patients with ulcerative colitis were given 2 to $\mathbf{4}$ grams (in divided doses) of Aerosol orally in enteric coated capsules daily. The fecal lysozyme activity fell from 130 to 170 units per gram to 13 to 20 units per gram within 48 hours. The 24 hour lysozyme excretion fell from levels of 51,800 to 110,000 units to an activity of 6,134 to 10,375 units, an inhibition of 84.7 to 88.2 per cent.

An Isotopic Study of Porphyrin and Hemoglobin Metabolism, in a Case of Porphyria. Morses Grinstein, RoBERT A. AldRICH and Violet Hawkinson (Introduced by C. J. Watson), Minneapolis, Minn.

An unusual opportunity has been afforded to study the formation of uro- and coproporphyrin in a case of light sensitive (so-called congenital) porphyria. In this case, a girl of 4, large amounts of uroporphyrin I and smaller amounts of coproporphyrin I are excreted in the urine, and the reverse in the feces. The same porphyrins have been isolated in crystalline form from the circulating erythrocytes, and have been demonstrated in lesser amounts in the plasma. Applying the method of Shemin and Rittenberg, $15 \mathrm{gm}$. of glycine containing 32 atoms per cent in excess of nitrogen 15, were administered orally in divided doses over a 3-day period. Serial determinations were then made of the $\mathrm{N}_{15}$ content of crystalline proptoporphyrin from the circulating hemoglobin, and of the crystalline uro- and coproporphyrins isolated from the urine and feces, as well as the crystalline stercobilin from the feces. Probably due to the fact that this patient also had a severe anemia with markedly increased erythropoiesis, the uptake of $\mathrm{N}_{18}$ by the hemoglobin protoporphyrin was considerably larger during a comparable period than that previously recorded for normal subjects. Similarly, high concentra- 
tions of $\mathrm{N}_{15}$ were found in the uro- and coproporphyrin isolated during different periods. The data will be discussed in relation to hemoglobin metabolism.

The Neuromuscular and Ganglionic Blocking Action of bis-Trimethylammonium Decane and Pentane Dibromide ( $C 10$ and $C$ 5). David Grob and Duncan A. Holaday (by invitation) and A. McGehee Harvey, Baltimore, Md.

Paton and Zaimis studied the effects in animals of poylmethylene bis-trimethylammonium salts of the general formula $\left(\mathrm{CH}_{8}\right)_{8}-\mathrm{N}-\left(\mathrm{CH}_{2}\right)_{\mathrm{n}}-\mathrm{N}-\left(\mathrm{CH}_{8}\right)_{3}(\mathrm{Br})_{2}$. They found the $\mathrm{C} 10$ derivative $(\mathrm{n}=10)$ to be a neuromuscular blocking agent and the $C 5$ derivative $(n=5)$ an autonomic ganglionic blocking agent. The administration of these compounds to man has shown that they are very effective in their blocking action at these sites and are clinically useful. The intravenous injection of $2.5 \mathrm{mg}$. of $\mathrm{C} 10$ in normal subjects resulted in almost complete paralysis of the muscles of the extremities, with relatively slight weakness of swallowing, speech, and respiration. C 10 was administered to more than 60 patients for the relaxation of skeletal muscles during convulsive shock therapy and surgical anaesthesia. The effective dose of $\mathrm{C} 10$ was one-third that of d-tubocurarine on a weight or molar basis, and it had relatively less effect on the bulbar and respiratory muscles. The action of $\mathrm{C} 10$ was of shorter duration than that of d-tubocurarine. C 10 caused a progressive decline in the muscle action potential in response to nerve stimuli. In contrast to d-tubocurarine it produced no histamine-like effects. C 5 produced evidence of autonomic ganglionic block in doses one-tenth to one-twentieth that of tetraethylammonium, on a weight or molar basis. The intravenous administration of 5 to $20 \mathrm{mg}$. of $C 5$ resulted in marked postural hypotention in most normal and hypertensive subjects, and a prompt fall in blood pressure in some hypertensive subjects. These effects persisted for as long as 3 to 6 hours.

The Role of the Kidney in the Pathogenesis of Malignant Hypertension as Indicated by a Study of Dogs Maintained with an Artificial Kidney after Nephrectomy, Implantation of the Ureters into the Bowel or Vena Cava, and Ligation of the Ureters. ARTHUR Grollman, E. E. Muirhead and John Vanatta (Introduced by Gladys Fashena), Dallas, Texas.

That the kidney plays a role in the animal economy in addition to its recognized function as an excretory organ has been inferred, particularly in relation to hypertension. However, direct experimental evidence bearing on this problem has not been possible since nephrectomy or abolition by other means of renal function leads to death from excretory insufficiency before other evidences of renal dysfunction are manifested. The present study is based on observations of more than 150 dogs subjected to (1) bilateral nephrectomy, (2) bilateral ligation or severance of the ureters, or (3) implantation of one ureter into the small bowel or into the vena cava with subsequent removal of the contralateral kidney. Survival of the animals was extended by application of an artificial kidney, the use of which shall be described. Nephrectomy results in an elevation of blood pressure and pathological changes in the kidneys characteristic of malignant hypertension. These observations, which are contrary to those generally held on the basis of short survival studies, offer direct evidence against the view that renin or other pressor substances play a role in the pathogenesis of hypertension.

In the presence of renal tissue as when a ureter is implanted into the duodenum or vena cava and the contralateral kidney removed, no elevation in blood pressure ensues. However, pathological changes induced by the accumulation of catabolic waste products from the organism are induced. The experiments permit certain conclusions as to the relation of the presence of renal tissue, blood pressure changes and uremia in the pathogenesis of the structural changes observed in malignant hypertension.

\section{The Application of Electron Microscopy to the Study of Connective Tissue Constituents. JEROME Gross (In-} troduced by Walter Bauer), Boston, Mass.

The electron microscope because of its high resolving power is capable of revealing the form of aggregation of macromolecules in certain biological systems. When this organization is of a constant and regular pattern one is provided with an accurate fingerprint of the substance which can be used for identification and study of formation and alteration.

The fibrous components of the connective tissue, collagen and elastin, are particularly suitable subjects. Up to the present only normal tissues have been studied, in part for the purpose of providing a base line for the eventual examination of pathological material. An extensive study of collagen from many tissues of many animals has revealed a characteristic fibrillar unit with an axial repeating period of $640 \mathrm{~A}$ units and a distinctive pattern of intraperiod fine structure.

A study of the elastic tissue of the mammalian aorta, ligamentum nuchae and fish swim bladder has revealed characteristic non-banded branching fibers which when digested by trypsin give rise to long filaments $120 \mathrm{~A}$ in width in the form of tight, evenly coiled helices produced by the interlacing of finer threads approximately $70 \mathrm{~A}$ in width. The pitch of the helix, approximately $530 \mathrm{~A}$ was the same for filaments from human, rabbit and rat aorta as well as ligamentum nuchae. Boiling the tissue in dilute acid to remove collagen prior to enzyme digestion did not affect the filaments.

The influence of $\mathrm{pH}$ and temperature on the state of aggregation and helical morphology of these structures will be discussed.

It is concluded that the elastic fiber is a two component system composed of bundles of trypsin-resistant filaments of characteristic form and size imbedded in a trypsinsensitive, heat-resistant "amorphous" binding matrix. The possible relationship of the elastic properties of the tissue to this structure will also be discussed.

The Effects of Sympathectomy and Other Sympathicolytic Procedures on the Cerebral Circulation in Essential Hypertension. JOSEPH H. HAFKeNSCHIEL and 
Henry A. Shenkin (by invitation), Seymour S. Kety and William A. Jefrers, Philadelphia, $\mathrm{Pa}$.

Quantitative data on blood flow, oxygen consumption and vascular resistance of the brain were obtained in hypertensive patients before and after bilateral lumbodorsal sympathectomy (9 patients), differential spinal sympathetic block (17 patients), parenterally administered dihydroergocornine ( 7 patients) and bilateral stellate ganglion block ( 5 patients).

Cerebral vascular resistance was significantly decreased following thoracolumbar sympathectomy, differential spinal block and intramusclar dihydroergocornine. Cerebral vascular resistance was not lowered after block of the stellate ganglia bilaterally.

These findings are interpreted as indicating that the increased resistance in the brain of essential hypertension is partially reversible. No evidence has been adduced that this resistance is mediated through the stellate ganglia, but our data do not rule out completely a neurogenic mechanism. It is of interest that the cerebral blood flow and cerebral oxygen metabolism are unchanged with the hypotension of sympathectomy and dihydroergocornine; the cerebral blood flow decreased significantly only when the blood pressure was acutely and severely reduced by differential spinal block.

It is concluded that procedures which produce a prolonged but moderate fall in arterial blood pressure in hypertensives do not bring about a sustained reduction of the cerebral blood flow when measured in the supine position since the vessels of the brain are capable of compensatory dilatation. When the reduction in blood pressure is sudden and excessive, however, the compensation may be incomplete.

Analysis of Mechanism of Cooley's Anemia by Transfusion Survival of Erythrocytes. HENRY E. HAMILTON and Raymond E. Sheets (Introduced by Elmer L. DeGowin), Iowa City, Iowa.

The survival of normal erythrocytes and those from patients with Cooley's trait were studied after transfusion into normal subjects, persons with Cooley's trait, and patients with Cooley's anemia by the Denstedt modification of the Ashby differential agglutination technique. A new method of analysis was employed in which the ratio of the concentrations of the donor's cells to those of the recipient was used to indicate fluctuations in the plasma volume of the recipient, the relative rate of disappearance of transfused erythrocytes, the abnormal destruction of the recipient's cells, and temporary erythropoietic arrest.

It was found that normal erythrocytes normally survive transfusion into subjects with Cooley's trait and Cooley's anemia. The red blood cells from persons with Cooley's trait persist normally in the circulation of healthy subjects and in subjects with Cooley's trait. They also survive a normal span in patients with Cooley's anemia. Analysis of the donor-recipient-cell ratio demonstrates that the mechanism of anemia in Cooley's disease is the rapid destruction of the patient's own erythrocytes. Similar hemolytic episodes of milder degree were also noted in persons with Cooley's trait. In addition, periods of diminished and augmented rates of erythropoiesis were noted in normal subjects, persons with Cooley's trait, and patients with Cooley's anemia.

(The data will be presented from twelve blood transfusions in which the method was applied in the study of Cooley's anemia. Controls are derived from many other transfusions studied in normal subjects and patients with various blood dyscrasias.)

Effect of Influenza Viral Infection on Ciliary Movements in the Mouse. CARL G. HARFORD and (by invitation) Mary Hara, St. Louis, Mo.

Cells of respiratory epithelium of man and other animals are equipped with cilia and the movements of these hair-like structures are thought to be an important means of natural resistance to bacterial infection. One theory concerning inception of respiratory infection is that viruses destroy the cilia-bearing cells of the epithelium thereby enabling bacteria to gain a foothold. In previous experiments, it was found that lowered pulmonary resistance of the mouse to inhaled pneumococci is associated with the lesion due to infection with influenza virus. The purpose of the present study was to determine whether viral impairment of ciliary action in the bronchi contributes to this lowered resistance since influenza virus is known to attack the bronchial epithelium.

In addition to special stains for demonstration of cilia, their movement was visualized directly in thin slices of fresh lung which were mounted as wet preparations in saline. When the margins of bronchi were viewed under the microscope with reduced light or by phase microscopy, the rapid ciliary beat was seen clearly.

In the viral lesion of 5 days duration, it was found that regeneration of bronchial epithelial cells had taken place and the cilia appeared normal both in morphology and motility. Therefore, in order to observe the maximal effect of viral infection on ciliary action, many lethal doses of virus were employed and observations were made after 2 to 3 days so that the greatest number of epithelial cells would be in the stage of necrosis. Even under these conditions, the majority of ciliated cells were not involved and profuse ciliary movement was observed regularly.

These findings do not support the theory that viruses affect resistance by interference with ciliary action.

\section{Liver and Kidney Function in Rocky Mountain Spotted}

Fever. George T. Harrell, Winston-Salem, N. C.

Chemotherapy or specific immunotherapy is administered to eradicate an etiologic agent. Specific therapy, as opposed to supportive therapy, may not prevent or control physiologic disturbances induced by an infection. From 1942-1947, liver and kidney function tests were performed on 16 children and 10 adults with Rocky Mountain spotted fever. Tests were done on admission, at the clinical peak of the disease, during convalescence and after discharge.

Liver: Less than $3 \mathrm{gm}$. of galactose was excreted in 11 tests. In 21 tests, less than $2.7 \mathrm{gm}$. (oral) or $0.7 \mathrm{gm}$. (IV) of hippuric acid was excreted in 13 instances. 
Bromsulfalein was retained 6 times in 18 tests. Serum bilirubin exceeded $4 \mathrm{mg}$. per cent only once. Prothrombin time was elevated markedly in 2 patients. Serum albumin was below $3.5 \mathrm{gm}$. per cent in 18 patients.

Kidney: Urea clearance was below 70 per cent in 9 of 13 patients. Phenolsulfonphthalein excretion exceeded 65 per cent ( 50 per cent in children) in 9 patients. The NPN exceeded $40 \mathrm{mg}$. per cent in 7 patients. Slight albuminaria was occasionally observed. RBC were found in the urine in 5 patients and casts in 8.

Liver damage, related in degree to the clinical severity of the disease, was greatest by hippuric acid test and occurred with or following the clinical peak. BSP, prothrombin, and albumin alterations occurred during the acute febrile phase. Function returned to normal with convalescence.

Kidney damage was less marked in degree, unrelated to the clinical severity, occurred before or during the clinical peak and returned to normal with convalescence. No glomerulonephritis was seen. Azotemia is probably due to fluid and circulatory disturbances rather than renal damage.

In 18 patients a high protein diet protected against changes in serum albumin and hippuric acid excretion; it did not decrease renal function but occasionally overloaded the kidney.

The mechanism responsible for the changes is obscure.

The Response to Adrenocorticotrophic Hormone in Patients with Scleroderma and the Therapeutic Use of Testosterone. SAUl Hertz and (by invitation) PETER H. ForshaM, Boston, Mass.

Scattered evidence has pointed to an endocrine factor in scleroderma, but none put forth to date incriminates any specific glandular defect. Suggestive features of a positive type include the marked incidence of hypercreatinuria, reduced creatine tolerance tests, low urinary 17ketosteroid excretion and a relationship of onset of periods of exacerbation to menstruation, menopause, etc. Predominance of the disease in the female sex, and in prepubertial males, has been impressive. Disorders of calcium metabolism (ectopic calcification), negative nitrogen balance and marked wasting have been emphasized. We have confirmed these findings in our series. The creatinuria, myopathy, negative nitrogen balance, pigmentation and occasional low serum sodium and chloride levels are compatible with some degree of gonadal or adrenal hypofunction. That this might be secondary to pituitary underactivity is suggested by low FSH titres, I ${ }^{\text {sn }}$ uptake by the thyroid and 17-ketosteroid excretion.

Seven cases have been studied from the standpoint of 17-ketosteroid excretion, creatine tolerance and responses to epinephine and ACTH.

17-Ketosteroid values on 24-hour urines in this group ranged from $1.2 \mathrm{mg}$. to 6.0 . However, 48 -hour tests with ACTH (40 mg. per day) led to a marked rise in 17ketosteroid excretion and other evidences of " $S$ " factor activation.

Since ACTH is not available for therapeutic application, we chose to observe 4 patients on high dosage of tes- tosterone propionate intramuscularly. Dosage ranged from 25 to 50 mgs. $\times 3$ per week. Gradual disappearance of skin lesions, as well as improvement in esophageal involvement (radiologic evidence) took place. Rapid weight gain and increased appetite and strength together with the establishment of a positive nitrogen balance occurred. Therapy was continued to the point of development of edema; slight hirsutism and masculinization of the voice were encountered. These subsided quickly after cessation of therapy and the skin lesions showed no recurrence four to five months after the end of therapy.

The Pulmonary Vascular Resistance. JoHn B. HickaM (Introduced by Eugene A. Stead, Jr.), Durham, N. C.

Normally the pulmonary arterial pressure is low and little affected by changes in blood flow. In congestive failure the pressure is high and may be greatly increased by exercise without change in flow. It is difficult to interpret these observations in terms of the state of the pulmonary vessels because the pulmonary arterial pressure also depends on the blood flow and the pulmonary venous pressure. The latter can not usually be measured. The present report presents data obtained from 4 patients with atrial septal defect in whom it was possible to catheterize both pulmonary arterial and venous systems and to measure blood flow, pressure gradient, and pulmonary vacular resistance.

In 2 subjects without apparent pulmonary vascular disease, the resistance was extremely low $(0.6$ to $0.9 \mathrm{~mm}$. $\mathrm{Hg} / 1 . / \mathrm{min}$.). Blood flows of 15 and $201 . / \mathrm{min}$. were maintained by gradients of 13 and $12 \mathrm{~mm}$. Hg. One subject with congestive failure and pulmonary arterial hypertension had a flow of $15 \mathrm{1} . / \mathrm{min}$. with a gradient of only 4 $\mathrm{mm}$. Hg. This indicates that the high pulmonary arterial pressure resulted from transmission of a high pulmonary venous pressure back through the pulmonary vascular bed. The low gradient suggests passive dilatation of the bed. Exercise caused a large rise in pulmonary arterial pressure, two-thirds of which resulted from an increase in venous pressure and one third from an increase in vascular resistance. One subject had pulmonary vascular disease and a high resistance ( 80 times that of the preceding subject).

The observations provide quantitative data on the wide potential range of pulmonary resistance and suggest the means by which congestive failure produces pulmonary arterial hypertension.

Experimental Evidence on the Mechanism of Diabetic Ketosis. Lawrence E. Hinkle, JR., and Grorge A. CONGER (by invitation) and StewART Wolf, New York, N. Y.

In a study of 25 human subjects with diabetes mellitus, approximately 50 instances of clinical ketosis were observed to occur in a setting of emotional conflict and in the absence of other pertinent factors including infection. Moreover, day to day observation of these subjects both in and out of the hospital yielded a close correlation between life situation, emotion, and the metabolic state as 
reflected by glycosuria, ketonuria, insulin requirement, and the symptoms of diabetes.

In an experimental study of nine of the subjects, quantitative measurements of blood ketone and glucose concentrations and concomitant determination of urine volume and glucose were made before, during, and after an interview in which intense emotional conflict was engendered. Ages of the patients varied from 14 to 60 years, and insulin requirements from none to 100 units per day. The chemical determinations were made on either perpheral venous blood or on blood withdrawn directly from a catheter introduced into the hepatic vein. In all cases a significant elevation of blood ketones, as well as a marked increase in the urine volume and rate of urinary glucose excretion, occurred during the traumatic interview. In the most severe diabetics the rapidity and degree of the increase in blood ketones and urine sugar was greatest, but nevertheless a marked degree of ketosis was produced in one of the mildest diabetics when the traumatic conflict situation was prolonged. The level of the blood glucose also fluctuated significantly, and was usually lower at the end than at the beginning of the experimental period.

Thus, significant emotional conflict has been shown to be associated with a rise in the blood ketone level and a simultaneous "washing out" of glucose through diuresis. The evidence indicates that such a mechanism is commonly involved in the decompensation of diabetes and the production of clinical ketosis.

The Usefulness of the Prompt Direct Reacting (1') Serum Bilirubin in the Study of Mild or Latent Jaundice. F. W. Hofrbauer, Minneapolis, Minn.

During the last four years the fractional serum bilirubin determination according to the Ducci-Watson modification of the Malloy-Evelyn method, has been widely applied in the study of patients with hepatic, biliary tract, and hematologic disorders. The method is informative at any level of the serum bilirubin, but has demonstrated greater usefulness in mild or latent jaundice. Therefore, for the purpose of the present study, the cases included have had total serum bilirubins not exceeding $3 \mathrm{mg}$. per $100 \mathrm{cc}$.

The level of the prompt reacting (1') serum bilirubin has been very helpful in demonstrating hepatic abnormality or low grade biliary obstruction where the total serum bilirubin was increased but slightly or not at all. In a number of instances of minimal regurgitation jaundice, the increase in prompt reacting serum bilirubin was well correlated with bromsulfalein retention and positive cephalin cholesterol flocculation (parenchymal liver disease), and with increases of alkaline phosphatase and serum cholesterol (extrahepatic biliary obstruction). Conversely, in pure retention jaundice, increases in the indirect reacting $\left(T-1^{\prime}\right)$ serum bilirubin, with slight or no increase in the $1^{\prime}$ value, have been quite uniform.

Data correlating the essential clinical features and the results of other liver function studies will be analyzed and discussed.

Experimental Evidence on the Relative Effects of Life Stress and Inhaled Pollen in Hay Fever. Thomas H.
Holmes and Theodore $F$. Treuting (by invitation) and Harold G. WolfF, New York, N. Y.

An experimental attempt was made to clarify the relative importance of stressful life situations and inhaled pollens in the pathogenesis of hay fever. In 30 subjects, 15 of whom had had hay fever, repeated quantitative studies of nasal function were made under uniform conditions in a room in which a known concentration of pollen was circulated.

Nasal hyperfunction characterized by varying degrees of hyperemia, swelling and hypersecretion of the membranes as well as eosinophilia in the nasal secretions and circulating blood occurred in all subjects, "sensitive" or "normal," exposed to mixed ragweed pollen when the circumstances were appropriate. When nasal function was average, neither "sensitive" nor "normal" subjects reacted to mixed ragweed pollen with sufficient evidence of nasal hyperfunction to produce symptoms. However, when there was preexisting nasal hyperfunction from whatever 'cause, both groups reacted to the pollen with marked nasal hyperfunction, weeping and sneezing. Thus during difficult life situations productive of conflict typical hay fever attacks followed pollen inhalation. Conversely, it was possible during pollen inhalation in the absence of frank hay fever to induce an attack by a discussion of significant personal problems and to induce subsidence of the attack by reassurance while the pollen is still being inhaled.

Unilateral procaine block of the stellate ganglion in 10 subjects yielded evidence that nasal hyperfunction engendered by conflict situations was mediated through parasympathetic fibers in the greater superficial petrosal nerve. Inhalation of pollen following this procedure precipitated the signs and symptoms of unilateral rhinitis which spared the non-hyperfunctioning membrane on the uninjected side. It was concluded that "sensitive" individuals differ from normals only in degree, that pollen or stressful life situations may induce nasal hyperfunction in either with associated local and circulating eosinophilia and symptoms of rhinitis. The various factors provocative of nasal hyperfunction with symptoms of hay fever thus exert an additive effect.

Quantitative Estimate of Vasomotor Tone in the Human Extremity with Comparative Studies of the Sympathetic Blocking and Adrenolytic Properties of Tetraethylammonium, Priscol, and Dihydroergocornine. Sibley W. Hoobler, John W. Avera, Samuel G. McClellan and William J. Little (Introduced by Richard $\mathrm{H}$. Lyons), Ann Arbor, Mich.

Presumably complete blockade of sympathetic vasomotor tone in the human subject without marked organic vascular disease was produced by means of spinal anesthesia, caudal anesthesia, lumbar paravertebral block, or within 24 hours after sympathectomy. Blood flow to the foot, as measured by the venous occlusion plethysmograph, was regularly increased to an average of 14 times control values. In contrast, tetraethylammonium (500 milligrams intravenously) increased blood flow to an average of 7 times resting levels and produced no vasodilatation after sympathectomy or after intra-arterial administration. In 
usual clinical doses it therefore produced an approximately 50 per cent blockade of sympathetic vasoconstrictor tone and had no local vasodilator action.

Priscol (30-50 milligrams intravenously) induced a three fold increase in blood flow in the foot and a two fold increase in the innervated and denervated hand. Blood flow likewise was increased after intra-arterial injection. In this dosage the effects of the drug can in large measure be accounted for by a local vasodilator action, although slight sympatholytic activity can not be excluded.

Dihydroergocornine (0.25 - 1 milligrams intravenously) caused a slow and delayed increase in blood flow to 2.6 times resting levels in the foot and 2.1 and 2.4 times resting levels in innervated and denervated hand respectively. In this dosage the drug has little or no sympatholytic effects and may produce vasodilatation after conversion into a vasodilator agent or by altering existing vaso-regulatory mechanisms which are not mediated through sympathetic pathways.

Adrenolytic activity was measured by determining the effect of the drugs on the vasoconstrictor response to intra-arterial injection of epinephrin. When tetraethylammonium, DHO, or benzodioxane (16 milligrams) was administered intravenously, the response to intra-arterial epinephrin was not altered. On the other hand priscol intravenously had a moderate adrenolytic effect. When high local concentrations of the last three drugs were achieved by intra-arterial injection, significant adrenalin blocking action was demonstrated.

Iron Metabolism Studies in Normal Subjects and in Patients having Blood Dyscrasias. REX HUFF and Thomas Hennessey (by invitation), and JoHN $\mathrm{H}$. LAwrence, Berkeley, Calif.

Shemin and Rittenberg have provided a method for accurate determination of the life of the red cell. With the need in mind for a method of determining the rate of production of hemoglobin; iron turnover in plasma and red cells of normal subjects and patients having polycythemia, leukemia, and refractory anemia has been studied. Total subjects numbered 30 .

Iron 59 (80-480 micrograms) was administered intravenously. The plasma iron level was not significantly altered; thus the clearance as measured by the rate of decrease of labeled iron in the plasma was effected at a constant plasma iron concentration. The coefficient of clearance (fraction of plasma volume cleared of iron per hour) was as low as 0.09 in a refractory anemia patient and as high as 3.84 in a polycythemia patient. The normal value was 0.35 .

The appearance of iron 59 in the circulating red cells was usually of a double exponential nature. The time required for a final level to be reached varied from 5 to 40 days. The trend was toward a more rapid appearance in the polycythemics and some of the leukemics.

The fraction of the total dose present in the circulating red cells at equilibrium was computed on the basis of a circulating red cell mass determined by tagged cell methods or by estimation. The fraction utilized varied from 0.05 in a refractory anemia patient to 0.95 in a patient who had had a severe hemorrhage. The average normal was $\mathbf{0 . 8 0}$.

The amount of iron incorporated into red cells per day varied from $220 \mathrm{mg}$. in a polycythemic to $2.9 \mathrm{mg}$. in a refractory anemia patient. The average normal value was approximately $20 \mathrm{mg}$.

The authors believe that here is a method for the determination of the rate of hemoglobin production in normal and pathologic states.

The Role of the Hypothalamus in the Pituitary-Adrenal Cortical Response to Stress. David M. HuME (Introduced by Francis D. Moore), Boston, Mass.

The mechanism responsible for increased secretion of anterior pituitary adrenocorticotrophic hormone after nonspecific trauma and stress, and the consequent rise in the titre of circulating adrenal cortical hormone has been studied in dogs and man. Trauma, insulin, chemical irritants, and epinephrine have been used as the stimulators of the pituitary-adrenal cortical system. The fall in circulating eosinophils has been used as an indicator of increased adrenal cortical activity, as this has been shown previously to be a measure of 11-17-oxysteroid output by the adrenal cortex, and to be dependent on the secretion of anterior pituitary adrenocorticotrophic hormone. The integrity of the anterior pituitary and adrenal cortex has been shown to be essential for the eosinopenic response to stress.

Anterior hypothalamic lesions in the supra-optic nucleus producing diabetes insipidus do not interfere with the normal eosinopenic response to stress; nor does divesting the pituitary of its vascular and nervous connections with the intact hypothalamus. However, specific localized electrolytic lesions placed in another area of the anterior hypothalamus abolish the usual eosinopenic response to stressing agents, even in the presence of an intact pituitary; and significant alterations in response follow lesions in the afferent tracts to this area. An extract of the hypothalamus has been prepared which on injection is capable of producing good eosinopenic responses in normal animals and also in animals whose hyperthalamic lesions render them incapable of responding to any of the stressing agents tested. These findings suggest the presence of a hormonal mechanism located in the anterior hypothalamus which, when activated in stress, will release a substance capable of causing an increased secretion of adrenocorticotrophic hormone by the anterior pituitary. In the absence of this mechanism no increased secretion of adrenal hormone can be detected in response to stress by the methods employed.

Electrolyte and Renal Function Studies in Lower Nephron Nephrosis. Lloyd T. Iseri, Thomas M. Batchelor, A. J. Boyle, and S. D. JACoBson (by invitation) and Gordon B. Myers, Detroit, Mich.

With the aid of the Beckman flame photometer and emission spectrograph, rapid determinations of plasma and urinary sodium and potassium were obtained and utilized as a guide to therapy in patients with lower nephron nephrosis. Simultaneous mannitol, para-aminohippurate. 
sodium and potassium clearances were determined periodically. Impaired tubular reabsorption of sodium with relatively high urinary sodium concentration was demonstrated during the early stages and restoration to normal reabsorptive capacity during recovery. Markedly negative nitrogen balances were also observed during recovery.

In some of the cases, renal functional studies were correlated with the pathological findings in material obtained by renal biopsy or at autopsy. The relationship between pre-renal azotemia, lower nephron nephrosis, and bilateral cortical necrosis of the kidneys will be discussed.

Studies of the Principle in Liver Effective in Pernicious Anemia. VII. The Augmentative Effect of Accessory Factors on the Therapeutic Activity of Vitamin B12. Bernard M. Jacobson and (by invitation) Ronald C. Bishop, Boston, Mass.

Evidence has accumulated that the therapeutic action of liver extract in pernicious anemia depends upon the presence of a primary factor, exerting by itself only slight or moderate hematopoietic effect, and upon the presence of five chemically distinct accessory factors which, in the absence of the primary factor, are therapeutically inert, but which augment the activity of the primary factor. The accessory factors include 1-tyrosine, a peptide, xanthopterin, tryptophane, and guanosine.

The availability of crystalline vitamin B12 made possible the study of this material as a primary factor. Erythrocyte regeneration curves were constructed from data in the literature and from personal observations concerning sixteen patients who received vitamin B12 parenterally in a calculated average daily dose of 2.1 micrograms. When contrasted with similar curves representing the effect in a larger number of patients of commercial liver extract in a calculated average daily dose of 2.1 U.S.P. units, it becomes evident that the hematopoietic effect of pure vitamin $\mathrm{B} 12$ is inferior to that of a comparable dose of commercial liver extract. Erythrocyte regeneration following the administration of a comparable amount of noncrystalline primary factor together with accessory factors similarly was more rapid than that induced by vitamin B12. Thus, in a range of initial erythrocyte levels from 1.50 to 1.99 millions per cu. mm., the average daily erythrocyte increment, in thousands per cu. mm., were as follows: commercial liver extract, 78; primary factor together with accessory factors, 86; vitamin B12, 59. Similarly, in a range from 2.50 to 2.99 millions per cu. mm., the average daily increment was 63,61 , and 31 , respectively.

The augmentative effect of accessory factors on the action of vitamin B12 is illustrated by data of individual cases.

The Role of the Spleen in Radiation Injury. LEoN O. JACOBSON, Chicago, Ill.

In an attempt to study the significance of the spleen in recovery from or compensation for radiation injury, four groups of mice were prepared. Group I consisted of untreated controls. Mice in groups II, III and IV were anaesthetized with nembutal and the spleens brought out through an abdominal incision with the main pedicle intact. Groups III and IV mice were irradiated with 600 $r$ whole body $X$ radiation ( $250 \mathrm{kv}$.) except that during the irradiation the mobilized spleens of group IV were placed in a lead box with an opening for the pedicle only. After irradiation the spleens of groups II, III and IV mice were returned to the abdominal cavity.

The widespread destruction of hematopoietic tissue which developed in mice that received $600 \mathrm{r}$ inclusive of the spleen (group III) produced a severe anemia, leucopenia and thrombocytopenia. Ectopic erythrocytopoiesis in the lead protected spleens of mice given $600 \mathrm{r}$ (group IV) compensated with such rapidity for the marrow destruction that no anemia of significance developed. Ectopic granulocytopoiesis and megakaryocytopoiesis in the lead protected spleens compensated significantly but at a slower pace and less completely for the marrow destruction.

A marked and sustained decrease in the amount of lymphatic tissue occurred in the lead protected spleens (group IV) as ectopic hematopoiesis increased. This decrease in lymphatic tissue may be an indirect effect of irradiation.

The rapidity with which erythrocytopoiesis transferred from the $X$ ray damaged marrow to the lead protected spleen in the absence of anemia suggests that the mechanism of stimulation of erythrocytopoiesis under the conditions of this experiment may involve some factor or factors other than or in addition to the accepted hemoglobin oxygen relationship.

Preliminary studies indicate that a significantly larger number of the irradiated animals with a lead protected spleen (group IV) survive than irradiated animals without spleen protection (group III).

Degradation of "Prothrombin-Converting Factor" in Sera of Individuals Receiving Dicumarol. RALPH F. JAcox and RoBert BAys (Introduced by Howard B. Slavin), Rochester, N. Y.

A study of the differential clotting action of fresh serum for fibrinogen and whole $0.01 \mathrm{M}$ oxalated plasma, reveals that the serum coagulating factor is not thrombin, but a "prothrombin-converting factor." This factor requires ionized calcium before it can be activated from an inert precursor existing in oxalated plasma or circulating whole blood; while the conversion of prothrombin to thrombin by the serum factor takes place in the virtual absence of ionized calcium. Immediately after coagulation has been initiated by thromboplastin or platelets, a residuum of "prothrombin-converting factor" remains in the expressed serum. In individuals with a normal quantity of plasma prothrombin, the rate of disappearance (or degradation) of the serum "prothrombin-converting factor" is constant. In sera of individuals receiving dicumarol, the degradation rate of the serum "prothrombin-converting factor" usually varies directly with plasma prothrombin concentration. By applying the formula

$$
\frac{\log c t_{1}-\log c t_{0}}{T_{1}-T_{0}}
$$


wherein $c t_{1}-c t_{0}$ is clotting time (seconds) of serum for plasma and $T_{1}-T_{0}$ is elapsed time (minutes) for a given change to take place, one can express degradation rate (D.R.) as a unit.

The sera and plasmas of a series of patients receiving dicumarol were assayed daily for prothrombin ( 1 and 2 stage technique) and serum "prothrombin-converting factor" degradation rate. Good correlation between the two determinations existed, although the measurement of the degradation rate of serum "prothrombin-converting factor" was no substitute for conventional methods of prothrombin assay.

Immunologic Studies of an Iron Binding Protein in $\mathrm{Hu}$ man Serum. B. V. JAGER (Introduced by M. M. Wintrobe), Salt Lake City, Utah.

The immunologic properties of a crystalline $\beta_{1}$ gobulin (Fraction IV-7-4) have been examined. This protein, isolated by Cohn and coworkers from plasma, has been found to have the property of binding with iron in vitro. A number of rabbits were immunized to this fraction. From a study of the antigen-antibody interactions of these antisera with the homologous antigen, Fraction IV-7-4 appeared to be immunologically homogeneous.

Standardized antisera were obtained by determining the amount of precipitate formed (antigen + antibody) after addition of varying known quantities of the IV-7-4 antigen. With this calibrated antiserum the amount of immunologically reactive IV-7-4 antigen in any unknown human serum can be determined by the quantitative precipitin technique.

As measured immunologically, the amount of IV-7-4 antigen in 14 normal human sera ranged from 0.21 to 0.31 grams per $100 \mathrm{ml}$. The mean value was 0.27 grams or 3.4 per cent of the total serum protein. Similarly, immunologic values were obtained for the sera of a number of pregnant women and a number of patients with acute or chronic infections. The values were increased in the pregnant women and reduced in the patients with infections. This was in accord with the alterations in the chemically determined total iron binding capacity of these sera.

The data are interpreted as indicating that the IV-7-4 protein present in human serum comprises all or nearly all of the iron binding protein.

Metabolic Changes in Heat Acclimatized Men during Abrupt Exposure to a Very Cold Climate. ROBERT E. Johnson and Chauncey G. Bly (by invitation), RobERT M. Kark and (by invitation) C. Frank ConsolAzIo, Chicago, Ill.

Thirty-two healthy young men, acclimatized for six months to Florida weather and unaccustomed to cold weather and its hardships, were taken by air from Florida (mean temperature $+57^{\circ} \mathrm{F}$.) to a very cold climate without layover, in January. On arrival in the subarctic, they were isolated in "survival areas." Studies were intensive for the first 24 hours (mean temperature $-26^{\circ}$ F.), and less intensive for 11 more days. Two questions were asked. What metabolic changes are manifest in untrained, unacclimatized men during abrupt exposure to extreme cold, even with adequate clothing and environmental protection? For such changes can unifying explanations be found in the literature on cold and heat acclimatization, the catabolic phase after injury, or the "adaptation syndrome"?

Statistically significant responses during the first 24 hours included : transient hypothermia; diuresis, with negative water balance; hemoconcentration, with increases in serum protein, hemoglobin and hematocrit; eosinophilopenia and lymphopenia; neutrophil leukocytosis, with increase in the percentage of immature forms; hyperuricemia, hyperphosphatemia and hyperkalemia; hyponatremia and hypochloremia; transient retention of sodium and chloride, with transient hyperkaluria; and diminution of ascorbic acid in the blood and increase in the urine. Most of these changes reverted to normal in 48 hours. Statistically significant responses in the last six days of exposure included: increase in kidney and adrenocortical efficiency, a test dose of water being excreted rapidly at very low specific gravity; continued eosinophilopenia and lymphopenia; and continued hyperuricemia and hyperphosphatemia. Complete balance data yielded no convincing evidence of a catabolic phase.

The present changes have many similarities to those after injection of the adrenocorticotropic hormone, and to the "general adaptation syndrome" of animals during stress.

Immunological Studies on Patients with Pneumococcal Pneumonia Treated with Penicillin. William S. JoRDAN (Introduced by John H. Dingle), Cleveland, Ohio.

Immunological studies of patients and animals with pneumococcal infections have shown that the naturally occurring production of antibodies is not affected by therapy with sulfonamide drugs. Meager data are available for patients treated with penicillin.

The immune response of 44 patients with pneumococcal pneumonia treated with penicillin was measured by agglutination and mouse protection tests. The results indicated that the antibody response of these patients was comparable to that occurring after spontaneous recovery or recovery following treatment with sulfonamides.

Two patients, one infected with type VIII and one with type I pneumococci, were treated within 2 and 3 hours of the acute onset of symptoms respectively. Neither patient developed a demonstrable pulmonary lesion or showed a rise in antibody titer. No other cases treated this early were observed. Of two cases treated within 7 hours of acute onset, both developed mouse protective antibodies and one developed agglutinins. Twenty-four cases received penicillin within 2 to 28 hours of the acute onset of symptoms. Nineteen of the 24 cases, or 79 per cent, showed demonstrable changes in antibody titers. This percentage response is comparable to that which occurred in the patients treated later than 28 hours after onset and parallels the response previously observed in patients not treated with penicillin. 
Correlation of Changes in Renal Circulation with Metabolic Balances of Electrolytes and Nitrogen during Recovery from Congestive Cardiac Failure. A. Kartus, A. Genecin, J. H. Sisson, C. Monge, B. C. SinclatrSmith, and E. V. Newman (Introduced by Alan M. Chesney), Baltimore, Md.

Two edematous patients with congestive cardiac failure were followed during recovery on bed rest and digitalis with metabolic balances of sodium, chloride, potassium and nitrogen. Periodic determinations of renal plasma flow (RPF) and glomerular filtration rate (GFR) were made.

One patient was observed for four months, 24 days on metabolic balances. There was negative sodium and chloride balance for 14 days, the weight and venous pressure falling to normal. Potassium and nitrogen balances were markedly positive during negative sodium balance. More potassium was retained than could be theoretically associated with the nitrogen stored, possibly indicating repair of cellular damage incurred during congestive cardiac failure.

GFR remained within normal limits throughout the four months of observation. RPF rose and filtration fraction (FF) fell during recovery. Three months later after reaccumulation of edema, GFR remained normal, RPF had fallen and FF had risen.

Another patient showed marked sodium chloride loss in four days, the weight falling 4 kilograms, followed by nine days of sodium chloride retention without increase in weight. Equilibrium was then established. Exercise daily for the next 11 days caused seven days of sodium chloride retention, followed by equilibrium. There was potassium and nitrogen equilibrium throughout.

GFR was 50 per cent and RPF 33 per cent of normal with an average FF of 0.40 throughout the balance study. Later the patient's rhythm was reverted from auricular fibrillation to sinus rhythm whereupon GFR rose from 80 to $100 \mathrm{cc} / \mathrm{min}$.

It is concluded that major changes in the body balances of sodium chloride and water may occur at both normal and reduced glomerular filtration rates. In each patient major shifts in salt balances, weight and venous pressure were not accompanied by significant change in glomerular filtration rate. The edematous state was associated with a high filtration fraction due to a low renal blood flow.

\section{Factors Influencing Removal of Bacteria from the Blood} Stream. Grace P. Kerby, Bernard C. Holland and SAMUEL P. Martin (Introduced by W. C. Davison), Durham, N. C.

Factors influencing the removal of bacteria from the blood stream have been studied effectively by a method of combined venous catheterization and bacteremia induced by continuous bacterial infusion. The sites of removal of the bacteria are determined in the anesthetized dog from the bacterial counts of samples of blood removed simultaneously from femoral artery and from veins draining the organs studied.

The major removal of bacteria is accomplished in the splanchnic circulation, the liver and spleen showing equal efficiency in the process. Removal during hind limb circulation may be of minor importance. No organisms are lost on circulation through the lungs.

Splanchnic removal rate is markedly and significantly decreased for a short period of time by thorotrast blockade.

The basic efficiency of the normally functioning removal mechanism varies widely with the bacterium used, being much greater in the dog for Escherichia coli and Micrococcus aureus than for Klebsiella pneumoniae. Arterial levels of 400 bacteria per ml. are reduced to 100 per ml. with each circulation through the splanchnic area. Sustained bacteremias clear within 15 to 30 minutes after bacterial infusion is discontinued.

Active immunization of the animal increases significantly the removal rate of Klebsiella pneumoniae. Administration of normal homologous plasma to the normal dog does not alter removal rate, but administration of equal amounts of homologous immune plasma results immediately in a striking increase in efficiency of splanchnic removal.

These alterations in efficiency of removal of bacteria from the blood stream unquestionably influence the ultimate course of the bacteremia. The extent of possible alteration and the exact mechanisms by which the removal is achieved and altered in the splanchnic area offer fruitful possibilities for further study. The present work points again emphatically to the importance of consideration of both parasite and host factors in any such continued investigation.

Studies in Protein Metabolism with the Aid of $S^{25}-\mathrm{La}$ beled-Methionine. Laurance W. Kinselz, Shelton Margen, Harold Tarver, Julie McB. Frantz and ERIN K. FlanaGaN; with the technical assistance of Vernon T. Thompson and Robert V. Deal (Introduced by T. L. Althausen), Oakland, Berkeley and San Francisco, Calif.

$S^{23}$-labeled-methionine has been administered to normal males and to patients with the following entities: (1) Chronic Liver Damage, (2) Idiopathic Hypoproteinemia, (3) Cushing's Syndrome. Incorporation of the labeled material in plasma protein, as well as its excretion in urine and stool, have been quantitated (in most instances as one part of protein balance studies).

From the results obtained, one may tentatively conclude that both the idiopathic hypoproteinemia syndrome and Cushing's syndrome are characterized by a more than normal rate of initial plasma protein formation (as evidenced by incorporation of $S^{25}$ ) followed (certainly in the former and probably in the latter) by excessive catabolism, at a rate such that net protein deficiency results.

The three patients with chronic liver damage so far studied show a diminished rate of incorporation of the labeled material into plasma protein, and a subsequent rate of disappearance not unlike that observed in the normal.

Urinary sulfate excretion data are in excellent accord with the preceding observations.

Chloromycetin in the Treatment of the Acute Manifestations of Brucellosis. Vernon KNIGHT and Francisco 
Ruiz-SANChez (Introduced by Paul Reznikoff), New York, N. Y.

Twelve adult patients with acute manifestations of brucellosis have been treated with chloromycetin. All but one of these individuals were treated in Guadalajara, Mexico, and the majority of the infections were caused by $\mathrm{Br}$. melitensis. All of the patients were febrile and acutely ill when antimicrobial therapy was started. The pretreatment illness was less than 90 days in 8 of the patients and ranged from 7 to 12 months in the remainder.

The diagnosis of brucellosis was established by demonstration of bacteremia in six patients and by a significant elevation in antibody titer in the others.

Appropriate clinical, bacteriologic and serologic observations were made during the acute illness and during the ensuing three months.

In general, chloromycetin was administered for six or ten days, in daily dose of 50 to $100 \mathrm{mg}$. per $\mathrm{kg}$. while febrile, and $25 \mathrm{mg}$. per $\mathrm{kg}$. thereafter.

Clinical improvement after therapy was dramatic. In every case temperature and symptoms rapidly diminished in the first three days of therapy. The average duration of fever was only 2.0 days, except for one individual who made an unsatisfactory response after six days of treatment with low doses. When retreated with higher doses, his course was comparable to that of the other patients.

The chloromycetin results can be compared with results observed following the aureomycin treatment of brucellosis by Spink and his associates and by the present writers. The immediate response of the patients treated with chloromycetin was indistinguishable from the response observed after aureomycin therapy.

Relapse was not uncommon following short periods of therapy with either drug. In the chloromycetin-treated series, relapse occurred within six weeks of the cessation of therapy in five of the twelve cases. The effect of a considerable prolongation of therapy upon the incidence of relapse is under investigation at present.

The Effect of Radiation on Total Coproporphyrin Excretion. Henry J. Koch, JR., Timothy R. Talbot, JR., and Mrtcher BeRnstein (Introduced by Konrad Dobriner), New York, N. Y.

The rate of total coproporphyrin excretion in the urine and feces has been observed in several patients receiving either the 8-day half-life radioactive isotope of iodine or $x$-radiation. In conjunction with this study complete hemograms including serial red-cell counts, hemoglobin, white-cell counts, platelet counts, bleeding and clotting time, bone-marrow biopsy, and liver function tests have been followed.

Three-day aliquots of urine and feces have been extracted for total coproporphyrin content according to the methods established by Dobriner and Watson. The etioisomers I and III of coproporphyrin are to be differentiated in a separate study.

It has been noted that there is an increase in total coproporphyrin excretion following radiation. This effect has been observed in patients receiving radioactvie iodine and $x$-radiation given in therapeutic doses, the largest single dose being 112 millecuries of $I^{102}$. The increased excretion has been reflected in both the urine and feces and has occurred ten to fourteen days following the onset of irradiation.

Concomitant with this increase in coproporphyrin excretion, a decrease in total lymphocyte count and a transient decrease in platelet count have been observed.

Granulomatous Polyarteritis: Report of a Case with Intraand Extravascular Granulomas Combining Certain Morphological Features of Poylarteritis Nodosa and Acute Rheumatic Fever. J. P. Kulka, D. G. Freman and W. S. Clark (Introduced by William W. Beckman), New York, N. Y.

The relation between vascular and extravascular lesions is one of the major problems in the morphology of the rheumatic diseases. Of particular interest is the relation between the characteristic necrotizing angiitis of polyarteritis nodosa and the Aschoff bodies and other extravascular granulomatous lesions of rheumatic fever. While both human autopsy studies and animal experiments seem to point to an intimate connection between these two disease manifestations, no direct evidence has yet been produced to indicate whether they are the result of the same or of different types of tissue injury.

Observations are therefore reported on a case of bronchial asthma and poylarteritis nodosa which at autopsy showed all transitional stages from classical segmental panarteritic lesions to miliary intramural granulomas on the one hand, and from extravascular miliary granulomas identical with those in the vessels to lesions indistinguishable from typical Aschoff bodies on the other. These findings strongly suggest that the segmental angiitis and the focal granulomas are both produced by the same pathogenic mechanisms and provide additional evidence that polyarteritis nodosa and rheumatic fever may be expressions of the same fundamental disease process.

Effect of Rigid Na Restriction in Patients with Cirrhosis of the Liver and Ascites. Henry G. KUnKer, WILliam J. Eisenmenger and Edward H. Ahrens, Jr. (Introduced by Thomas M. Rivers), New York, N. Y.

The intake of $\mathrm{NaCl}$ was limited to less than $1 \mathrm{gm}$. per day in 13 patients with steadily accumulating ascites. Fluid formation in the abdomen was immediately stopped in 12 of the 13 patients. The patients readily tolerated such a low salt diet for more than 3 months with the exception of one patient who showed an elevated NPN initially. As much as $150 \mathrm{gm}$. of protein per day could be consumed on this diet. The 12 patients who ceased to form fluid were markedly improved by the treatment, chiefly because the loss of body protein through paracentesis ended. This resulted in a rise in the serum albumin level. However, ascites reformed in all but 3 of the patients when the $\mathrm{Na}$ intake was raised to normal levels at the end of 3 months and longer periods of therapy were necessary for complete improvement. The critical level of $\mathrm{NaCl}$ intake where ascites formation began was ap- 
proximately $1.3 \mathrm{gms}$. $\mathrm{NaCl}$ per day. Above this level the amount of ascites which formed was directly proportional to $\mathrm{NaCl}$ intake.

Detailed studies of nitrogen, $\mathrm{Na}$ and $\mathrm{K}$ balances were carried out in 3 of the patients before and after $\mathrm{Na}$ restriction. The patients were found to be in negative nitrogen balance prior to therapy chiefly because of the loss of ascitic fluid nitrogen. When this was stopped, they reverted to positive nitrogen balance. All the patients showed extremely low excretion of $\mathrm{Na}$ in urine prior to therapy and this was further reduced after the special diet was begun. Fecal $\mathrm{Na}$ levels were approximately 3 times the urinary values.

The conclusion drawn from these investigations is that rigid $\mathrm{Na}$ restriction is a valuable supplement to nutritional therapy for stopping the malignant downhill course of patients with rapidly accumulating ascites.

Studies of Water Storage in Liver Disease. II. The antidiuretic activity of the urine during acute infectious hepatitis. DanIEL H. LABBY (Introduced by Edwin E. Osgood), Portland, Ore.

In a previous study of 14 cases it was demonstrated that the acute phase of infectious hepatitis was marked by expansions of the thiocyanate fluid space, the plasma and blood volumes, depression of the plasma and urinary chlorides and increased water tolerance. Improvement in liver function with convalescence resulted in shrinkage of these fluid compartments and diuresis. Slight changes in total circulating proteins were detected. Eight additional cases of early hepatitis were selected to correlate the antidiuretic activity of the urine with the above fluid observations. $200 \mathrm{gm}$. male rats were given water (5 per cent of body weight) by stomach tube and injected intraperitoneally with $1 \mathrm{cc}$. of the patients dialized and concentrated urine per 100 grams of rat. The time required for the excretion of 50 per cent of the ingested water was taken as a standard assay end point; controls were run simultaneously by injecting water of the same $\mathrm{pH}$ intraperitoneally. The data obtained in each case indicate that in the early phase of acute infectious hepatitis at the time of maximum water storage and increased water tolerance the 50 per cent excretion time ranged from 155 to $360 \mathrm{~min}$ utes in comparison to control times of 80 to 110 minutes. With convalescence, contraction of the thiocyanate space, and diuresis, the antidiuretic titer of the urine decreased to control values. It is therefore concluded that the movements of body water occurring during acute infectious hepatitis are accompanied by alterations in the concentration of the antidiuretic principle in the urine; the potency of this principle being highest during periods of water storage in the acute stage and lowest during convalescence when excessive water storage is no longer demonstrable. Opportunity was also presented for conducting these studies during menstruation in the course of hepatitis and in a case of nonicteric hepatitis.

Evidence That Renal Sodium Excretion Is Controlled by Adrenal Cortical Activity and That Ingested Sodium May Displace Intracellular Potassium in Normal Sub- jects. Alexander Leaf (by invitation) and L. H. Newburgh, Ann Arbor, Mich.

The effects of great variation in sodium intake were studied in normal subjects maintained on fixed diets of adequate calories and protein with very low sodium and chloride content. At definite periods additional sodium was administered either as the chloride or citrate.

Salt restriction resulted in reduction in urinary sodium and chloride to minute amounts accompanied by increase in urinary nitrogen, urea, uric acid, potassium and phosphorus. Administration of sodium chloride or citrate caused high urine sodium with decrease in urine uric acid, potassium phosphorus, blood and urine urea, and positive nitrogen balance. The drop in blood urea was not accounted for by alteration in glomerular filtration rate but was explained by decrease in the rate of protein catabolism. A strongly positive potassium balance occurred simultaneously with the high sodium excretion that could not be accounted for by decreased protein catabolism.

All these changes are explained by alterations in adrenal cortical activity. The need to conserve body sodium was met by increased activity of the desoxycorticosterone-like hormone. An associated increase in protein-catabolic hormone activity was elicited also. Sodium administration abolished the need to conserve sodium and thus depressed adrenal cortical activity. This not only allowed a large urinary sodium excretion but also gave evidence of a marked decrease in activity of protein-catabolic hormone.

In all subjects sodium citrate administration caused initially a marked positive sodium balance. The degree of alkalosis was reduced by entry of large amounts of sodium into the cells with displacement of large amounts of potassium as evidenced by the strongly negative potassium balance.

Diagnosis of Carcinoma of the Liver, Biliary Tract, and Pancreas from Cytology of Duodenal Aspiration. $\mathrm{H}$. M. LEMon and W. W. Byrnes (Introduced by James M. Faulkner), Boston, Mass.

The infrequency of successful surgical therapy for primary carcinoma of the liver, extra-hepatic bile ducts, and pancreas reflects in many cases clinical inability to establish an early positive diagnosis of cancer. In this study careful cytological examination has been made of aspirated duodenal contents in an effort to supplement currently available diagnostic methods. Although no previous studies have been reported concerning neoplastic cells in duodenal secretion, we have found that criteria accepted as indicating cancerous cell exfoliation from other epithelial surfaces can be readily applied to this fluid, since large numbers of tumor cells as well as normal epithelial cells have been observed in the majority of our specimens from patients with early or late cancer.

All cases have been followed for at least six months after examination or until death. Three-quarters of the cancer cases have been verified pathologically, and a close similarity in morphology has been found between the exfoliated cells and those in the pathological sections from the tumor. No false positive errors have been made, although control observations have been carried out in an equally 
large number of cases of cirrhosis and inflammatory condition of the biliary tract and pancreas. Among the diagnosed cancers were hepatoma, primary cancers of the gall bladder, common bile duct, Ampulla of Vater, and the head and tail of the pancreas. Localized carcinomas as small as $2 \mathrm{~cm}$. in diameter have been detected, as well as certain metastatic neoplasms. A brief description will be given of certain cases in which earlier diagnosis and institution of proper therapy have resulted from the application of this method to clinical problems with and without obstructive jaundice.

\section{Acute Asphyxia in Dogs: Effects on Blood Coagulation and Citric Acid Metabolism. Jessica H. Lewrs (In- troduced by E. C. Hamblen), Chapel Hill, N. C.}

These experiments were designed to study the effects of acute asphyxia on blood coagulation in dogs. The trachea was clamped in 13 anaesthetized dogs. Following the cessation of labored respiratory movements at about two and one-half minutes, the blood pressure fell, reaching zero at approximately six minutes. Two cc. arterial whole blood specimens were collected every fifteen seconds for the first two minutes and every five seconds thereafter. Markedly prolonged clotting times or incoagulable bloods were obtained during a $30-45$ second interval occurring about 30 seconds after respirations had ceased. Both before and after this period blood coagulation times were normal.

Blood coagulation studies on the "incoagulable blood" showed normal (1) prothrombin complex, (2) fibrinogen, (3) platelets, (4) fibrinolytic enzyme system, and (5) antithrombin. No heparin or defect correctable by added thromboplastin could be detected.

The coagulation defect was readily reversed by the addition of calcium chloride. In spite of this, the total calcium concentration was normal. Therefore, we reasoned that calcium must be bound in some soluble, but non-ionized form.

Anaerobic carbohydrate metabolism may produce citric acid. Studies on four dogs have shown a rise of citric acid concentration from a normal value of approximately $75 \mathrm{micrograms} / \mathrm{cc}$. to $1,000 \mathrm{micrograms} / \mathrm{cc}$. in the "incoagulable blood" followed by a fall to normal values at death. Aconitic acid did not change appreciably. Parallel studies with added sodium citrate showed that 900 micrograms/cc. (as citric acid) were sufficient to prevent coagulation.

Acute asphyxia in the dog produces a marked rise in blood citric acid content, sufficient to bind ionized calcium, and so far as we are aware, this is the first reported incidence of calcium ion deficiency resulting in a blood coagulation defect. An adequate explanation for the extremely temporary but marked elevation in blood citric acid is being sought.

Studies in Urinary Corticosteroids by a Method Permitting Analysis of Desoxycorticosterone. CharLes W. LLOYD and JuLIA LOBOTSKY (Introduced by Eugene L. Lozner), Syracuse, N. Y.
The method of Daughaday, Jaffe and Williams for estimation of freely water soluble urinary corticosteroids has been modified to permit analysis of the poorly water soluble corticosteroids, such as desoxycorticosterone.

Excretion of corticosteroids by normal individuals and by six patients with Addison's disease has been studied by this method. Low corticosteroid values were found in all patients without treatment. Following beginning of desoxycorticosterone acetate injections, there was a latent period of several days before the excretory rate began to rise. In one patient with a fixed daily dose of 5 milligrams of desoxycorticosterone there was a gradual increase in corticosteroid excretion over a period of a month. Following complete withdrawal of desoxycorticosterone, excretion continued at a high level for approximately 96 hours before a significant decrease was observed. In one patient, clinical evidence of adrenal insufficiency appeared within one day of this decrease. The excretion rate of corticosteroid was in the middle of the normal range when the desoxycorticosterone dose was at the optimal level as determined by clinical response. Excessive dosage was associated with higher corticosteroid values, which, however, did not exceed the normal range. Following implantation of pellets, with good clinical control, excretion of corticosteroid was lower than when desoxycorticosterone in oil was injected in amounts sufficient to produce a comparable clinical effect.

One patient with Addison's disease when untreated had a corticosteroid excretion only slightly below the normal range. Administration of desoxycorticosterone produced relatively small increases of urinary corticosteroids. Withdrawal of desoxycorticosterone was followed by the very slow appearance of symptoms of insufficiency. It is believed that this patient has viable adrenal tissue which is capable of secretion, although at an inadequate rate, and it is suggested that when desoxycorticosterone was administered, this secretion was depressed.

Studies in Hemoglobin Formation with the Aid of the Isotope Technique. IRving M. London, David ShEMIN and D. RitTenberg (Introduced by Randolph West), New York, N. Y.

When the whole blood of patients with sickle cell anemia is incubated in vitro with glycine labeled with $\mathrm{N}^{16}$, heme containing $\mathrm{N}^{15}$ is formed. This finding demonstrates in vitro synthesis of heme. No significant synthesis is found on incubation of normal human blood. To help determine whether the immature reticulocyte or the mature pathologic cell of sickle cell anemia is responsible for the synthesis, studies have been carried out in a variety of hematologic disorders characterized by elevated reticulocyte counts (pernicious anemia, congenital hemolytic jaundice, acquired hemolytic anemia) and in experimental animals in whom reticulocytosis was induced by bleeding or phenylhydrazine hemolysis. These studies indicate that some, though perhaps not all, reticulocytes are capable of synthesizing heme in vitro. The differences in capacity for heme synthesis manifested by reticulocytes of various sources will be discussed.

The incubation of $\mathrm{N}^{25}$ labeled glycine and other isotopic 
compounds with blood containing numerous reticulocytes affords a method for the study of heme formation. Avian nucleated red blood cells may be used similarly. Studies concerned with the optimal conditions for in vitro heme synthesis, with the use of the in vitro systems for the determination of the biologic precursors of protoporphyrin, and with some of the mechanisms involved in heme formation will be discussed.

Experimental Airborne Influenza Virus $A$ Infection in Immunized Animals. ClaytoN G. Loosli and (by invitation) Robert S. Rockwald and Merle H. RitTer, Chicago, Ill.

Young adult Swiss mice (10-15 grams) were immunized by 4 weekly $0.1 \mathrm{ml}$. subcutaneous injections of formalized mouse lung suspension (10-1 dilution) of $P R-8$ strain of Influenza $A$ virus. Neutralization and CRC agglutinin-inhibition tests were employed for antibody determinations on pooled sera. Immunized and nonimmunized mice of similar age were exposed in a chamber at the same time to a lethal atmosphere of nebulized mouse lung PR-8 virus suspension. Five mice from each group ( 2 for histology and 3 for virus and antibody titration) were bled and killed at close intervals until death or 16 days after inoculation. Additional animals served to determine survival rates.

In the non-immunized animals, the influenza infection was rapidly fatal with death occurring on or before the fifth day. The lungs at death showed extensive consolidation with marked destruction of the bronchial epithelium and extensive edematous and cellular exudate in the alveoli. The immunized animals became moderately ill but survived. Grossly, only focal areas of consolidation occurred, which underwent resolution after 8 days. Microscopically, as in the control lungs, there was extensive destruction of the bronchial epithelial cells but the alveolar exudate was patchy and generally confined to regions near the terminal bronchioles. Also, as in the non-immunized animals, the virus grew rapidly in the lungs of the immunized animals, reaching a titer of over $10^{\circ}$ lethal intranasal doses at 36 and 48 hours. At this time, the serum antibody level of 1:512 before infection had dropped to the non-immune level of $\langle 1: 16$. At 6 days, only traces of virus were present and the antibody titer had risen several fold above the original immune level. The mechanism of protection against influenza virus in the immunized animals and in man in relation to the above observations will be discussed.

Spirocheticidal Antibodies in Syphyilis. Harold J. MagNuson and (by invitation) Fred A. Thompson, Chapel Hill, N. C.

The in vitro technic described by Nelson, employing the Nichols strain of $T$. pallidum, permits demonstration of a spirocheticidal substance found in most syphilitic sera from humans and rabbits but not present in normal sera. Electrophoretic fractionation of such sera shows the activity to be associated with the gamma globulin. The substance is found in Fraction II-III of Cohn, is relatively heat stable, and requires complement for its demonstration. This evidence suggests that the substance is an antibody.

The antibody is not related to reagin since its presence bears no relation to the titer of serologic tests for syphilis. Sera from which reagin has been absorbed retain their activity, and the purified reagin recovered from the precipitate is inactive. Assays are reported on sera from 200 patients with syphilis in various stages as well as from rabbits with known varying degrees of immunity. The possible relationship of this antibody to acquired immunity is discussed.

The Production of Renal Glomerular Lesions in the Diabetic Rat. George V. MANN and James W. Goddard (Introduced by Howard F. Root), Boston, Mass.

Weanling male rats have been made diabetic by the intravenous injection of alloxan monohydrate. A total of 50 animals have been maintained on one of 4 diets up to 550 days. The diets were dog chow and semipurified diets containing 12, 18 and 40 per cent protein. No insulin was given. At intervals animals were sacrificed and tissues examined.

It has been found that diabetic rats develop well marked progressive lesions in the renal glomeruli as early as 50 90 days. The lesions primarily affect the reticulin walls of the glomerular capillary tufts, parietal layer of Bowman's capsule and tubular basement membranes.

Reticulin proliferation produces intercapillary adhesions and luminal obliteration. The latter process may occur either by collapse reticulosis or by luminal ingrowth of 'reticulin fibers. In some instances hyaline-like balls are entrapped in the inter-capillary and axial spaces. Reticulin overgrowth in a meshwork pattern leads to ball formation, reminiscent of the fibrous balls of human nephrosclerosis. The parietal layer of Bowman's capsule shows irregular thickening and collagenization. Tubular basement membranes show thickening with hyaline changes.

Especially in old diabetic animals an increased cellularity of the glomerular tufts appears due to a remarkable hyperplasia and hypertrophy of what appear as perithelial cells.

These lesions have not been found in nondiabetic control rates or in nondiabetic alloxan injected controls. No lesions have been found in the aorta and its branches. Renal arteriolar lesions have been minimal. Neither the severity of diabetes nor the level of protein in the diet has been shown to influence these manifestations.

Mechanism of the Depressor Effect of Accelerated Sodium Depletion in Hypertension. R. S. MEGIBOw, J. J. BooKMan, J. Sirota and H. Pollack, New York, N. Y.

We have shown that the depressor response developing in hypertensive patients on low sodium diets may be accelerated materially by increasing the rate of sodium depletion through the use of mercurial diuretics.

The following studies were undertaken in order to elucidate the more fundamental relationships involved.

A : Adrenocortical function was investigated from determinations of the 17 keto and 11 oxy corticosteroids, 
from glucose tolerance tests, from the blood uric acid, and from the eosinophile and lymphocyte response.

B : The effects of sodium depletion upon glomerular and tubular function and upon renal blood flow were determined by measuring the para-amino-hippurate and the mannitol or inulin clearances.

C: Using the T-1824 method, the possible role of decreased circulating blood volume in the depressor response to sodium depletion was studied.

D: The time relationships between sodium depletion and the depressor response were clarified by measuring the daily 24-hour sodium excretion by the flame photometer method.

E: Alterations in vascular reactivity were evaluated microplethysmographically, by contrasting the vasodilator responses to tetraethylammonium and dihydroergocornine before and after accelerated sodium depletion.

The evidence suggests that the decline in blood pressure which follows accelerated sodium depletion is associated with a temporary alteration of corticoid function. The conclusions drawn from the above investigations will be illustrated and discussed more completely.

\section{Clinical Studies on the Pure Veratrum Alkaloids Proto- veratrine and Veratridine. EDWARD MEILMAN and Otro Krayer (Introduced by S. L. Gargill), Boston, Mass.}

The vasodepressor and cardiodecelerator action of protoveratrine hydrochloride has been studied for the first time in human renal and essential hypertension. It was administered intravenously, eighty times in fifteen patients, in single doses of between $0.08 \mathrm{mg}$. and $0.18 \mathrm{mg}$.

The characteristic response is a fall of systolic and diastolic blood pressure reaching minimum level within ten minutes. Intensity of effect is roughly proportional to the dose, varying between a significant decrease and a blood pressure fall to normal levels. Duration of action, likewise, is proportional to the dose, return to hypertensive levels occurring between fifteen minutes and three hours. Repeated administration of the same effective dose, in the same patient, leads to similar responses after the previous effect has worn off.

The vasodepressor action is accompanied by bradycardia, usually with regular sinus rhythm. Occasionally, the largest doses shifted the pacemaker, the ventricle beating faster than the auricle.

Atropine reduces but does not abolish the vasodepressor effect, while it increases the heart rate above normal and restores regular sinus rhythm. Simultaneous administration of protoveratrine and atropine causes a vasodepressor effect with a concomitant increase in heart rate.

Apart from the disturbance of rhythm, a slight and transient feeling of nausea occurred a few times.

Veratridine has circulatory effects qualitatively similar to those of protoveratrine but is less potent. With veratridine significant vasodepressor action cannot be obtained without side effects like nausea, vomiting, sweating, and feeling of apprehension. Occasionally, these occurred with doses not decreasing blood pressure.
Their unique mechanism of action makes the Veratrum alkaloids important substances for careful clinical studies in hypertension. For satisfactory clinical use the search must be directed towards pure alkaloids pharmacologically similar to protoveratrine, with a margin of safety and an intensity and duration of vasodepressor action equal or possibly superior to that of protoveratrine.

\section{The Determination of Maximum Composite Digital Vas- cular Caliber Physiologically in Normal and Hyperten- sive Subjects. Milton Mendlowitz, New York, N. Y.}

In animal perfusion experiments with Ringer's solution the pressure-flow relationship has been demonstrated to be rectilinear. For blood this relationship has been found to be logarithmic. That portion of the curve above flow readings of one-third the "normal," however, corresponds closely to a rectilinear tangent with an intercept on the pressure coordinate above zero.

The pressure-flow relationship in the human digital circulation after release of sympathetic tone was studied at various pressure levels achieved by graded compression of the brachial artery. This relationship corresponded well with a rectilinear tangent both in normotensive and hypertensive subjects. The intercepts were similar to those observed in animal experiments.

The "apparent viscocity" of normal blood at a "normal" perfusion pressure has been shown in animals to be 2.2 times that of Ringer's solution. There is reason to believe that this holds for the human digital circulation. If the intercepts are known, it is possible to draw a pressure-flow tangent from a single pressure-flow reading. At the point of "normal" pressure a theoretical Ringer's solution perfusion point can be erected and the Ringer's solution pressure-flow line drawn to the intercept zero. Poisseuille's law is now applicable, the only unknown quantities being length and caliber of the perfused vessels. Since the flow is measured per sq. $\mathrm{cm}$. of skin, the average length of the perfused vessels can be estimated at $1.1 \mathrm{~cm}$. The composite digital vascular caliber of a single vessel, the resistance of which is equal to that of all the vessels per sq. $\mathrm{cm}$. of skin perfused can be computed. It was found to vary from 67 to 75 micra normally and to be below normal in hypertension.

Clinical Experience with the Use of an Artificial Kidney. J. P. Merrill (by invitation), G. W. ThORN, and (by invitation) E. J. Callahan and S. Smith, Boston, Mass.

A modification of the Kolff type of artificial kidney has been used in 28 cases in 18 patients with uremia. Difficulties encountered by previous observers with hemolysis, clotting and pyrogen reactions have been virtually eliminated. Adjustment of the tonicity of the bath fluid to the patient's serum by means of freezing point determinations has so greatly increased the accuracy with which hydration may be controlled that it is feasible to employ the procedure in uremic patients with congestive failure and even pulmonary edema. The adjustment and balance of blood flow rates through the machine have further de- 
creased its risk in application to patients with cardiovascular disease.

The effectiveness of this machine in removing diffusible substances from the blood by in vivo continuous dialysis is indicated by the fact that with blood flow rates between 200 and $300 \mathrm{cc}$. per minute and blood urea nitrogen level of 80 to $90 \mathrm{mg}$. per $100 \mathrm{cc}$., it is possible to remove 20 grams of urea per hour with comparable quantities of other retained metabolites. The machine may also be employed to remove specific ions by dialysis and three instances of acute spontaneous potassium intoxication have been successfully treated by the removal of excess potassium from the blood stream. The possibility of removing specifically other diffusible substances, including sodium, uric acid, water and amino acids is under investigation. It is concluded that the present technique of application of an artificial kidney is an effective and clinically safe procedure for the removal of diffusible substances by continuous dialysis of the blood. The value of its use as a means of therapy in acute renal failure and in a variety of forms of intoxication is well established. Its use as an investigative tool in the study of chronic cardiovascular and renal diseases is apparently warranted.

Treatment of Pernicious Anemia with an Animal Protein Factor of Bacterial Origin. LEO M. MEYER, NoRTON D. Ritz, Manuel Rowen, George Bock and Julius Rutzky (Introduced by Arthur C. De Graff), New York, N. Y.

An animal protein factor concentrate was prepared from the aerobic fermentation of a non-motile gram negative bacillus isolated from chicken feces. One cc. of this product (A.P.F. 60) had a biologic activity equal to "10 unit liver" when administered to chicks receiving a diet containing 70 per cent soy bean meal. In the lactobacillus assay the material had a somewhat lower potency (65 per cent of "10 unit liver"). A more concentrated preparation (Normocytin) had a potency comparable to 10 micrograms of vitamin B 12 when administered to chicks maintained on a diet containing 70 per cent soy bean meal. Administration of A.P.F. 60 to 5 patients with pernicious anemia in relapse induced a satisfactory reticulocyte response in 4 instances, with an increase of $\mathrm{Hb}$ and R.B.C. in all cases. Eight other persons with pernicious anemia in relapse treated with Normocytin showed a satisfactory reticulocytosis. An increase of $\mathrm{Hb}$ and R.B.C. to normal levels occurred in 6 cases. One patient from the original group treated with A.P.F. 60 was continued on Normocytin and also reached normal hematologic values. In all instances there was improvement in appetite and general well-being of the patients. Megaloblastic bone marrows were converted to normal states. Where neurological changes were present improvement was noted. There was no instance of progression or development of nervous system signs or symptoms. In two patients the $\mathrm{Hb}$ and R.B.C. remained below normal levels and are at present under treatment with vitamin B 12.

Effect of Urethane on the Susceptibility of Mice to Pneumonia Virus of Mice $(P V M)$. George S. MIRICK,
Charles I. Leftwich, Jr. and William B. Leftwich (Introduced by Paul W. Clough), Baltimore, Md.

The effect of urethane on the susceptibility of the young mouse to infection by pneumonia virus of mice (PVM) was tested. This compound seemed of interest because of its reported suppressive action on cell growth and its capacity to induce pulmonary tumors in older mice of certain strains.

Suitable matched groups of young mice were lightly anesthetized with ether, and inoculated intranasally with serial ten-fold dilutions of PVM. All groups were fed Purina Dog Chow and each was given to drink either water or water containing various concentrations of urethane. It was observed repeatedly that drinking 0.1 per cent or more urethane during the post-inoculation period rendered mice 5 to 10 times more susceptible to this virus infection than controls. A single intraperitoneal injection of urethane before virus inoculation produced similar effects.

Some possible mechanisms for urethane's action in increasing the mouse's susceptibility to PVM were studied. Mice given urethane orally gained less weight and drank less fluid than controls. However, limiting food or fluid in other mice did not reproduce the effect of urethane. Moreover, one injection of $5 \mathrm{mg}$. of urethane before inoculation increased susceptibility ten-fold without affecting the weight curve. Other cell growth depressants like colchicine and podophyllotoxin did not have effects like that of urethane on susceptibility to the virus infection.

\section{Insulin Tolerance Test in Patients with Essential Hy- pertension. I. Arthur MIRSky, (by invitation) Stan- LEY M. Kaplan and R. H. BroH-KaHN, Cincinnati, Ohio.}

The blood sugar response to the intravenous injection of a standard dose of insulin is dependent upon factors which affect the hypoglycemic action of insulin and factors which affect the restitution of the blood sugar to the preinjection level. Some of the hormones of the anterior pituitary gland and the adrenal cortex are among the factors which determine the effectiveness of the hypoglycemic action of insulin whereas the activity of the sympathicoadrenal system largely determines the rate of restitution of the blood sugar concentration to the preinjection level. Since both sets of factors have been implicated in the genesis and maintenance of the hypertension of patients with essential hypertension, we deemed it pertinent to study the insulin tolerance of such patients.

Blood samples were drawn for sugar determination before, and at 15, 30, 45, 60, 90 and 120 minutes after the intravenous injection of 0.1 unit insulin per kilogram body weight to fasting normotensive and hypertensive subjects. The carbohydrate stores of all subjects were fortified by ingestion of two grams of carbohydrate per kilogram body weight after the usual meal the night before the test.

The hypoglycemic response of both groups of subjects was not statistically different. However, statistical analysis of the data revealed that the rate of restitution of 
the blood sugar concentration to the preinjection level was markedly delayed in the hypertensive group.

These studies indicate that there is no significant increase in contra-insulin factors in patients with essential hypertension. Further, if the rate of restitution of the blood sugar concentration is dependent upon the degree of activity of the sympathico-adrenal system, then the response of this system to hypoglycemia must be reduced in the hypertensive group.

\section{The Destructive Effects of the Virus of Russian Far East}

Encephalitis on the Transplantable Mouse Sarcoma 180.

Alice E. MOoRE (Introduced by C. P. Rhoads), New York, N. Y.

A study was made of the ability of certain viruses to grow in transplantable mouse tumors and to determine what effect their growth would have on the viability of the tumor. The virus of Russian Far East Encephalitis was found to have a marked affinity for the transplantable mouse Sarcoma 180 and in the process of viral infection the viability of the tumor was completely destroyed. When subinfective amounts of virus were inoculated there was no effect on tumor growth and no virus could be recovered from the tumor or brain.

Following intraperitoneal inoculation of $0.05 \mathrm{cc}$. of $10^{-6}$ brain virus suspension, tumors were removed for bioassay and the amount of virus present in the blood, brain and tumor was determined. For the first five days more virus was present in the tumor than in either blood or brain. From the 5th day until the death of the animals approximately equally large amounts of virus were present in the tumor and brain while it disappeared from the blood. Portions of the tumors were implanted into virus-immune and non-immune control mice at different days after intraperitoneal inoculation. In all cases in which implantations were made into non-immune mice no tumor growth was apparent and the animals died of virus infection in 1 to 2 weeks. When pieces of these same tumors were implanted into virus-immune mice it could be demonstrated that although the virus was present in large amounts three days after intraperitoneal inoculation an additional 3 or 4 days exposure in the intact animals was necessary before all the tumor cells were killed.

Tolerance to the Toxic Effects of Somatic Antigens of Enteric Bacilli in Typhoid and Paratyphoid Fever Convalescents. HERBERT R. MORGAN and (by invitation) Frankiln A. Neva, Ann Arbor, Mich.

The somatic or $\mathrm{O}$ antigen (endotoxin) of Salmonella typhosa is the component responsible for toxicity of this organism and is a major determinant of its antigenic properties. When injected intravenously in man in minute doses, the endotoxin produces chills, fever, headache, muscle aching and generalized malaise and in some instances nausea and vomiting. Since there is evidence that the somatic antigen of $S$. typhosa is present in the blood during the acute phase of typhoid fever, it has been suggested that this toxic component might be responsible for certain of the clinical manifestations of the disease.
Following repeated intravenous injections of typhoid somatic antigen, individuals develop a resistance to its toxic effects which is not dependent on the presence of circulating $O$ antibody which also appears. This tolerance is also protective against the similar toxic manifestations of the serologically unrelated antigen of Shigella dysenteriae for which no $\mathrm{O}$ antibodies are present.

Patients convalescent from typhoid and paratyphoid fevers and certain other infectious, febrile diseases were tested for resistance to the toxic effects of somatic antigens of $S$. typhosa and Sh. dysenteriae. Twenty-five of thirty-one patients convalescent from typhoid fever and eighteen of twenty-one patients convalescent from paratyphoid fever showed no significant reactions to intravenous test doses of the endotoxin of $S$. typhosa $(5 \gamma)$ and Sh. dysenteriae $(1 \gamma)$ which produce marked febrile responses and systemic reactions in normal individuals.

When retested four to six months later, eight of eleven of these patients were shown to be resistant. In the three patients who were susceptible to the initial test dose, four test injections showed that the tolerance to the toxic effects of the somatic antigens was rapidly reestablished.

Five patients, who were convalescent from other febrile infectious diseases, developed fever and systemic reactions which were comparable to those seen in normal subjects following the injection of test doses of somatic antigens.

The implications of these findings in relation to recovery from typhoid and paratyphoid fever will be discussed.

\section{Plasma Volume, Red Cell Volume and "Extravascular \\ Thiocyanate Space" Changes in Patients on Rice Diet. \\ Richard J. F. MURPhy (Introduced by Julian M. \\ Ruffin), Durham, N. C.}

Serial observations of the plasma volume, "extravascular thiocyanate space" and red cell volume of 19 patients on the Kempner rice diet have been made. Period of observation was 14 weeks; measurements were made before starting the diet and at 3,6,10, and 14 week intervals following.

Seventeen patients had hypertensive disease of different types and 2 had coronary artery disease without hypertension. None had cardiac failure nor azotemia. Eleven had some alteration in renal function as measured by urinalysis, P.S.P. excretion and concentration test.

Plasma volume was measured by T1824 dilution and red cell volume was calculated from venous hematocrit.

Sixteen of the 19 patients showed significant loss of plasma volume. Mean loss in these patients was 14 per cent. This occurred chiefly during the first 6 weeks, the volume remaining relatively constant during the following 8 weeks observation.

In general loss of "extravascular thiocyanate fluid" followed the same pattern. The mean loss in this volume was 15 per cent.

Decrease in red cell volume was a constant finding in all 19 cases and showed no simple relation to fluid loss. The mean loss was 24 per cent. This could not be accounted for by blood loss from venesection for study purposes. In those cases with plasma loss only one-third 
of this was reflected in the hematocrit, the other twothirds being masked.

Weight loss was a prominent finding in some cases. There was no close correlation between weight loss and fluid loss either of degree or in time relationships. Although fluid loss was greatest in those with evident renal damage, 7 of the 8 patients with normal renal findings showed significant loss. There were no cases of frank clinical salt depletion.

There was no simple relationship between volume changes and clinical course.

Observations on the Excretion of Bromsulphalein. J. D. Myers (Introduced by Keith S. Grimson), Durham, N. C.

Two important factors in the excretion of bromsulphalein by the liver are (a) amount of dye presented to the liver per unit of time, i.e. hepatic plasma flow, and (b) ability of hepatic cells to extract and excrete dye. Some evidence concerning the operation of these factors in normal and diseased states has been collected while measuring hepatic blood flows. Pertinent data are the arterial concentrations of BSP resulting from various dosages of dye, and the percent extraction of dye by the liver. Since the corrected infusion rate of BSP can be assumed equal to hepatic excretory rate, the data allow calculation of the BSP clearance

$$
\text { infusion (excretion) rate }
$$

arterial concentration

as ml. of plasma cleared completely of dye per minute per sq. M. Mean BSP clearance in 39 control subjects was $244 \pm 83 \mathrm{ml}$.; mean per cent extraction was $53.2 \pm 13.7$.

In 8 patients with severe, chronic anemia the increased hepatic plasma flow, due both to increased whole blood flow and lowered hematocrit, provided a normal if not supernormal clearance $(277 \pm 135 \mathrm{ml}$.) in the presence of decreased per cent extraction $(30.7 \pm 13.7)$. In 19 patients with cardiac failure, and low cardiac outputs and hepatic blood flows, the decreased clearance $(89 \pm 43 \mathrm{ml}$.) was due to both decreased supply of dye to the liver and impairment in hepato-cellular function (mean per cent extraction $28.5 \pm 11.5$ ). Nine individuals with Iaennec's cirrhosis of moderate severity who had normal or moderately subnormal blood flows, had a markedly reduced mean clearance $(134 \pm 41 \mathrm{ml}$.) due largely to decreased extraction (32.8 \pm 15.6 per cent). In 16 patients with hyperthyroidism mean clearance $(212 \pm 97 \mathrm{ml}$.) and per cent extraction $(37.2 \pm 17.6)$ were not significantly different from control values, although 6 of the group did have subnormal extraction percentages and presumably hepato-cellular dysfunction.

In both controls and ill patients, simultaneous infusion of bilirubin, human albumin, or amino-acids with bromsulphalein impaired excretion of BSP by decreasing per cent extraction.

\footnotetext{
Incidence and Nature of Hepatic Disturbance Following Acute Viral Hepatitis and in Persons with Maximal and Minimal Exposure to Hepatitis Virus: A Prelimi-
}

nary Report. J. R. NEEFE and (by invitation) Hugo Dunlap Smith, Charles H. Kurtz, Lewis William Bluemle, Jr., S. Clay Williams and Join G. ReinHold, Philadelphia, $\mathrm{Pa}$.

The incidence and nature of hepatic disturbance has been investigated in: Group IA : 300 persons chosen at random from records containing sufficient information to justify a diagnosis of acute viral hepatitis 2 to 7 years previously; Group II (Maximal Exposure Group) : 158 persons chosen at random from records indicating unusual exposure to hepatitis virus during the preceding 7 years; Group III (Minimal Exposure Group) : 120 persons selected because of the lack of any recognized unusual exposure to hepatitis virus. Group IB is composed of selected patients with moderately severe symptoms and disability following acute viral hepatitis. The study of each person included a detailed history, a complete physical examination, a comprehensive group of hepatic tests, and, in representative cases, needle biopsies of the liver. An unexpectedly high incidence of findings suggesting mild hepatic disturbance has been encountered in both the maximal and minimal exposure groups. The incidence of such findings to date in the unselected post-hepatitis group (IA) has been only slightly higher. The observations on the selected post-hepatitis group (IB) support existing evidence of the occasional occurrence of a severe form of chronic liver disease with diffuse hepatic fibrosis following acute viral hepatitis. The data from the 3 "unselected" groups indicate that such major sequellae are infrequent but reveal the existence of a relatively large number of persons in all $\mathbf{3}$ groups with evidence of chronic mild hepatic disturbance which may be related to preceding apparent or inapparent infection with hepatitis virus.

Studies on Treponemal Immobilizing Antibodies Produced in Syphilitic Infection. RoBert A. NeLson, JR., Judith A. Diesendruck and Harold E. C. Zheutlin (Introduced by Thomas B. Turner), Baltimore, Md.

A method has been developed by which antibodies to virulent Treponema pallidum can be demonstrated in vitro. Treponemes are extracted from rabbit testicular syphilomas and suspended in a special medium in which the organisms remain motile and infective for several days. On incubation of such suspensions with syphilitic rabbit or human sera and guinea pig complement, the treponemes become non-motile and lose their capacity to infect rabbits. This immobilizing activity is virtually absent from normal serum.

Using this technique, a preliminary survey of serum and cerebrospinal fluid from patients in various stages of syphilis has been made. Of 20 sera from patients with primary syphilis, 10 produced marked immobilization, three were doubtful, and seven showed no immobilizing effect. All sera from 20 patients with secondary syphilis, 15 with symptomatic tertiary syphilis, and two with congenital syphilis were strongly positive. All sera from 20 presumably normal individuals, and 20 patients with diseases other than syphilis were negative. 
Similarly, cerebrospinal fluids from 10 patients with central nervous system syphilis were positive while fluids from 10 non-syphilitic patients were negative.

By absorption experiments it has been demonstrated that the immobilizing antibody is distinct from reagin which is detected by the ordinary serological tests for syphilis. Thus far, the sera of seven patients with unquestionable biological false-positive serological reactions were completely negative with the treponemal immobilization test.

Since the immobilizing antibody appears to be directed specifically against $T$. pallidum, it may afford a practical method for the study of the immunology of syphilis in man.

The Effects of Salt-Poor Albumin on the Excretion of Water and Electrolytes in Edematous Patients. JACK Orloff, Louis G. Welt and Lyman Stowe (Introduced by Paul H. Lavietes), New Haven, Conn.

The infusion of 100 grams of a 25 per cent solution of salt-poor albumin may initiate a diuresis of water and sodium in edematous patients. When albumin is administered to normal subjects, however, despite similar hemodynamic changes, the excretion of sodium is decreased and that of water not significantly altered.

Albumin was injected intravenously as a 25 per cent solution into 2 patients with toxemia of pregnancy, 3 with the nephrotic syndrome, an edematous pregnant female without toxemia and 2 normal subjects. In those patients in whom the sequence of events were analyzed, there was initially a profuse water diuresis in which water was lost far in excess of sodium. The resultant elevation of the concentration of sodium in the extracellular fluid was associated with a diuresis of salt.

In contrast, in both normal individuals and in edematous patients who did not develop a water diuresis during the infusion of albumin, there was no change in the level of sodium in the extracellular water, and no subsequent increase in the excretion of salt.

From these studies it appears that the primary diuretic action of albumin in some edematous patients is to promote a diuresis of water. If water is lost sufficiently in excess of salt to elevate the concentration of sodium in the extracellular compartment, an increased excretion of sodium ensues.

Observations on Experimental Pulmonary Edema. RoBert Paine (by invitation) and John R. Sмith, St. Louis, Mo.

Experimental heart failure in dogs with intact circulation usually causes no pulmonary edema, although pulmonary congestion may be marked. In contrast, heart-lung preparations always develop "spontaneous" pulmonary edema, in time. These phenomena were investigated in dogs. In all instances pulmonary lymph flow was measured by modifications of Drinker's technique.

In eight experiments, congestion of individual lung lobes, or of the entire lungs, produced no increase in lymph flow nor evidence of pulmonary edema.
The role of decreased plasma proteins in lung edema was investigated in nine experiments. Sufficient intravenous Locke's solution was infused to reduce the plasma proteins to about 2.6 grams per cent. Marked and sustained pulmonary lymph flow occurred, although systemic venous pressure was little influenced by the infusions. In addition, complete mechanical obstruction of the pulmonary lymphatic duct provoked no evident gross pulmonary edema; however, in the same animals Locke's infusion again resulted in lung edema.

Heart-lung preparations were set up, following isolation of the right lymphatic duct. Pulmonary lymph flow increased immediately after establishment of the heartlung circuit (five experiments). In eleven other instances, lymph flow was carefully measured as various vessels were tied off in making heart-lung preparations. Thus, heart-lung-head preparations could be established in which the lung lymph flow was not increased. However, when the cephalic arteries were ligated (making an ordinary heart-lung), augmented lymph flow and eventual pulmonary edema occurred. Possibly autonomic effects from cerebral ischemia may influence the development of pulmonary edema when the heart-lung-head is transformed to the heart-lung circuit. Other evidence suggests that the rapidity of onset of pulmonary edema in the heart-lung is intensified when left ventricular overloading occurs, producing elevation of both pulmonary arterial and venous pressures, and consequent destructive rise in capillary pressure.

An Abnormality in Nitrogen Metabolism in Cushing's Syndrome Altered by Testosterone Propionate: A Study Utilizing Glycine Tagged with Isotopic Nitrogen, $N$ 15. William Parson (Introduced by Thomas Findley), New Orleans, La.

Isotopic nitrogen, $\mathrm{N} \mathrm{15}$, was incorporated in glycine and fed in single small doses to healthy men on normal and high protein diets. The rates of urinary excretion of $\mathrm{N} 15$ were determined by the use of the mass spectrometer. Simultaneous nitrogen balances were made. Our results are similar to the observations of the Columbia group, reported in part by Sprinson. Approximately 30 per cent of the fed $\mathrm{N} 15$ appears in the urine in 24 hours when the diet contains 1 gram of protein per kilogram of body weight. The rate increases to approximately 45 per cent on a high protein diet containing 1.5 grams of protein per kilogram.

A patient with Cushing's Syndrome in nitrogen equilibrium and maintaining her weight on a diet containing 1 gram of protein per kilogram, was given "tagged glycine" as above. N 15 excretion rates were determined. The observations were repeated during a period of nitrogen retention induced by testosterone propionate. Control observations were again made when nitrogen equilibrium returned after cessation of therapy.

In this patient, at the end of 24 hours, approximately 45 per cent of the fed $\mathrm{N} 15$ appeared in the urine during both control periods. This figure fell to approximately 30 per cent during testosterone administration. 
The significance of this abnormality in nitrogen metabolism is not established. If the assumption is made that "tagged glycine" traces "protein metabolism," analysis of the data suggests that the abnormality in this case of Cushing's Disease may represent "decreased protein anabolism," altered toward normal by testosterone propionate.

Cerebral Blood Flow and Oxygen Consumption in Neurosyphilis. John L. Patterson, Jr., Albert Heyman and Fenwick T. Nichols, JR. (Introduced by Paul B. Beeson), Atlanta, Ga.

The cerebral blood flow was determined by the nitrous oxide technique of Kety and Schmidt in 48 patients with neurosyphilis and 12 control subjects. Cerebral oxygen consumption was calculated from the flow data and the arteriovenous oxygen difference.

In patients with paresis and meningovascular syphilis the cerebral blood flow was below the normal mean in almost every instance. The mean cerebral blood flow in these conditions was 75 per cent and the lowest 32 per cent of the normal mean. The cerebral oxygen consumption was reduced in both groups, but a more marked reduction was found in the patients with paresis. In these patients the average oxygen consumption was approximately 70 per cent of the normal with an extreme value of only 24 per cent of the normal. In patients with asymptomatic neurosyphilis, both the blood flow and oxygen consumption were within the normal range.

The effect of penicillin and malarial fever therapy was studied in 17 of the patients. The mean cerebral blood flow one to six months following treatment in paresis showed no consistent change, while the oxygen consumption showed a marked mean increase ( 35 per cent). These changes could be correlated to a considerable degree with improvement in the patients' mental status. In most of these patients the improvement in cerebral oxygen consumption was proportionately greater than the increase in blood flow. In meningovascular syphilis the mean cerebral blood flow following therapy increased by an average of 20 per cent of its pre-treatment value, while the oxygen consumption showed only a small increase. In several patients with either paresis or meningovascular syphilis the improvement in cerebral blood flow was associated with an actual decrease in oxygen consumption or vice versa.

These studies suggest that the syphilitic process can exert a direct effect on cellular metabolism in the brain in addition to impairing its blood supply.

Postoperative Alkalosis and Potassium Deficiency. 0. H. Pearson and L. P. Eliel (Introduced by Rulon W. Rawson), New York, N. Y.

A syndrome characterized by alkalosis, hypochloremia, and hypopotassemia has been observed in 15 patients undergoing major surgery and being maintained by intravenous feeding for several days postoperatively. Clinically these patients exhibited muscular weakness, drowsiness, nausea, abdominal distention, and cardiac irregu- larities. Electrocardiographic changes occurred, such as dopression of $T$ waves, prolongation of the $Q T$ interval, depression of ST segments, inversion of $P$ waves, frequent extrasystoles and auriculo-ventricular block. Administration of potassium chloride intravenously or by mouth resulted in clinical improvement, a return to normal of the acid-base and electrolyte disturbance, and disappearance of the electrocardiographic abnormalities.

These electrolyte disturbances show a striking resemblance to those observed in certain patients with Cushing's syndrome. Darrow has observed similar electrolyte changes in rats that were made potassium deficient by several different methods. We have observed the development of alkalosis and potassium deficiency in a patient treated with adrenocorticotrophic hormone. The data will be presented.

These results suggest that the pathogenesis of this syndrome in postoperative patients is due to the alarm reaction producing hyper-function of the adrenal cortex, with loss of potassium and chloride (or retention of sodium) in the urine, associated with a low intake of potassium. Other factors which may contribute to the loss of potassium and chloride will be discussed.

\section{A Statistical Analysis of the Physiological Factors De- termining Cardiac Output in Normal Subjects. RAY- mond Pearson (Introduced by William S. McCann), Rochester, N. Y.}

Heretofore, interest in cardiac output has been concerned with methods and results rather than relationships of the factors concerned. The determinants of cardiac output can be expressed by various equations relating heart rate, stroke volume, oxygen consumption, ventilation and arteriovenous oxygen difference. This study is a statistical analysis of the available data both in the literature and from our "Chest" Laboratory.

The results indicate that there is a linear increase in cardiac index from the basal state to that of severe exerrise and that the rate of change seems to be fairly constant despite the method used to determine it. For each 10 per cent increase in oxygen consumption there is a 7 per cent increase in cardiac index. The arteriovenous oxygen difference shows a gradual widening with exercise but this change is minimal constituting only about 10 per cent of the total increase. Heart rate increases more than stroke volume with an increasing cardiac output. Total ventilation is directly related to either heart rate or cardiac index during exercise. As oxygen consumption increases with exercise, approximately 90 per cent of the change is accounted for by increasing ventilation whereas only 10 per cent is made up by the increase in the volumes per cent oxygen absorbed from air. The oxygen transported per heart beat reflects more of a change in arteriovenous oxygen difference than in stroke volume. Slides are presented to illustrate these changes.

Clinical Study of 11-Dehydro-17-Hydroxy-Corticosterone in Hypertension, Addison's Disease and Diabetes. George A. Perers and (by invitation) Kermit L. 
Pines, Howard B. Hamilton ąd Katherine VisLocky, New York, N. Y.

The present study was undertaken in order to determine the clinical and metabolic effects of synthetic 11dehydro-17-hydroxy-corticosterone acetate (Cpd. E.) (Merck). Because of the possibility that this steroid might exert a depressor action on the arterial tension, in addition to its reported modification of carbohydrate metabolism, observations were made on four patients, two with uncomplicated hyertensive vascular disease, one with Addison's disease, and one with diabetes mellitus and hypertension.

Observations were made after an adequate baseline and on a constant regimen. Cpd. $E$ was administered intramuscularly (20 mgs. every 6 hours) for periods usually of eight days.

At least transitory salt and water retention was observed, but an increased nitrogen excretion was not consistently apparent. Serum electrolyte values were not materially affected. A slight decrease in cholestrol esters was noted. Urinary 17-ketosteroid and "corticoid" excretion did not change significantly. Blood sugars, after 17 hours' fasting, and glucose tolerance tests were minimally altered, but the results were of doubtful significance. Transitory ketonuria appeared in some subjects. Cpd. E produced an increase in total white cell count, together with a pronounced drop in eosinophiles. Percentage uptake of radioactive iodine in the patient with Addison's disease fell slightly.

Cpd. E exerted a depressor effect on the "resting" blood pressure of the hypertensive patients.

The Use of Intravenous Human Albumin in Decompensated Cirrhosis of the Liver. Josepr Post and (by invitation) JEROME V. ROSE, Bronx, N. Y.

Human albumin was administered intravenously to sixteen patients criticaly ill with decompensated cirrhosis of the liver, for about three months, in amounts sufficient to maintain normal serum albumin levels. The dietary regime consisted of about 3,500 calories with 140 grams of protein, and supplementary B complex parenterally. Two patients had post-necrotic cirrhosis and fourteen were alcoholics with Laennec's cirrhosis. Due to the gravity of illness, the pre-treatment observation period was one to six weeks.

Of these sixteen patients, thirteen improved and had complete diureses, two showed only widening of the paracentesis intervals, and one had no effect (later died with hepatoma). Of the thirteen patients who had diureses, five died some weeks later of the following causes: "cholemia"-2; hematemesis-1; carcinoma of stomach1 ; uremia-1. Seven patients who had diureses, were comatose when therapy was begun.

Six other patients, not included above, were in deep coma on admission and survived only two, two, three, seven, eight and thirteen days of therapy.

Thirty-two patients, with decompensated Laennec's cir- rhosis of the liver, were observed during a period of six to eight weeks for control studies, while on the above dietary regime. These patients did not appear as seriously ill as were those treated with albumin. Twentyone improved and diuresed without albumin treatment. Ten died for the following causes: "cholemia" -1 ; hematemesis- 5; uremia-1; hepatoma-2; intestinal obstruction-1.

Intravenous albumin has a place in the therapy of decompensated cirrhosis of the liver.

The Nature of Auricular Flutter. Myron Prinzmetal, and (by invitation) I. C. Brill, Elior Corday, Alvin L. Sellers, Walter A. Flieg and H. E. Kruger, Los Angeles, Calif.

The present concept of auricular flutter, based upon the classical studies of Lewis and his coworkers, is that this arrhythmia is due to a pure circus movement moving down one auricle and up the other, ringing the venae cavae. Lewis believed that daughter waves were emitted to the periphery of the auricles from the main circus path. By taking slow motion pictures (2000 frames per second) of fluttering auricles, (250 times slower than normal speed) the flutter wave can be readily seen. When flutter is produced by placing aconitine in the center of the surface of the right auricle it can be seen that the flutter contraction travels in all directions from the ectopic focus and not in one direction as would be demanded if Lewis's circus movement were the cause of flutter. No daughter waves of contraction are visible. A burn placed across the path of the theoretical circus wave does not interfere with the flutter. Flutter contractions appear similar to the contractions in paroxysmal tachycardia. The main difference is that flutter is due to more rapid discharge from the ectopic auricular focus with varying degrees of A-V block. Auricular flutter results when an ectopic focus discharges itself at a faster rate than in paroxysmal tachycardia but slower than in auricular fibrillation. The flutter wave does not pursue a circular course but travels in all directions from the site of impulse formation by all available routes.

\section{Differential Diagnosis of Adult Rheumatic Fever and Rheumatoid Arthritis by Means of Serological Tests. R. W. QUinN and S. J. LiAO (Introduced by John R. Paul), New Haven, Conn.}

Serological studies are reported on series of cases as follows: (i) 50 cases of active rheumatic fever; (ii) 42 cases of inactive rheumatic fever (both groups (i) and (ii) largely adults); (iii) 40 cases of acute beta hemolytic streptococcal upper respiratory infections; (iv) 35 adult cases of rheumatoid arthritis, and (v) 45 normal subjects. One to six samples of blood from each of these subjects have been studied by means of a battery of tests, including, antihyaluronidase, antistreptokinase, antistreptolysin "O," and streptococcal agglutination with both autoclaved and live organisms. 
The introduction of two relatively new tests, namely (a) antihyaluronidase and (b) Thulin's agglutination technique employing autoclaved beta hemolytic streptococci, are significant points in this study. Their use places the former (a) in relation to the other streptococcal antienzyme tests and the latter (b) in relation to conventional agglutination tests using live organisms.

Results of these tests indicate that in active rheumatic fever all of the antibody titers except the agglutination titers are significantly higher than in the other groups studied. In inactive rheumatic fever titers are not significantly higher than in normal subjects, except in the case of the agglutination titers which were higher than normal. In patients convalescent from upper respiratory hemolytic streptococcal infections, the titers of all four tests are higher than in those with inactive rheumatic fever, rheumatoid arthritis or normal subjects.

Similarities in the clinical pictures of adult rheumatic fever and rheumatoid arthritis are discussed along with the serological means now at our disposal for differentiating these two conditions. This discussion indicates that in any individual case, high titers in any one or all of the anti-enzyme tests and a high agglutination titer would seem to favor a diagnosis of rheumatic fever, whareas low anti-enzyme titers and a high agglutination titer would favor a diagnosis of rheumatoid arthritis.

Study of a Human Gystinuric Fed Radioactive Methionine. Lester J. Reed, Doriano Cavallini, Fred Plum, Julian R. Rachele and Vincent du VignEAUD (Introdued by Robert F. Watson), New York, N. Y.

Studies on human and experimental animals having spontaneous cystinuria have clarified some of the steps in organic sulfur metabolism. A source of urinary cystine sulfur has been proved to be ingested methionine in dogs through the use of radioactive sulfur $\left(S^{36}\right)$. The present communication extends this type of approach to the human.

Through the incidental finding of a spontaneous cystinuria of from approximately 0.3 to $0.5 \mathrm{gm}$. per day in a patient with a post-meningitis convulsive disorder, we were provided the opportunity to study this cycle. Radioactive sulfur $\left(\mathrm{S}^{25}\right)$ in methionine (200 mg.) was given this patient in an amount considered safe by the standards of the Health Physics Department at Oak Ridge, Tennessee.

Following the ingestion of tagged methionine, radioactive sulfur $\left(\mathrm{S}^{35}\right)$ was found in the cystine of this patient's urine within 24 hours, and later was detected in the cystine of her hair. This unequivocally demonstrated that cystine sulfur may arise from ingested methionine. Coincidentally with the feeding of the $S^{26}$ the patient had a transient increase in capillary fragility, fall in hemoglobin and decrease in red blood count. There had been an associated nutritional difficulty with the convulsive disorder. These abnormalities responded to iron therapy and the treatment of the primary clinical difficulty, and an 18 month follow-up has revealed no abnormality attributable to the radioactive sulfur.
The Action of Insulin on Glycogen Deposition in Adipose Tissue. Albert Renold and D. W. FAwcett (by invitation) and Alexander Marble, Boston, Mass.

The observations here reported were made during a study of lipodystrophies due to insulin. A promising line of approach was suggested by the report of Fawcett in 1948 who called attention to histological studies demonstrating under certain conditions considerable amounts of glycogen in fatty tissue.

In our studies the glycogen in the fat of diabetic rats was determined chemically and found to be negligible in untreated animals whereas amounts as large as 1.5-3.0 per cent of the fresh weight of fat could be demonstrated 24 hours after a single large dose of insulin. This action of insulin could be shown to be both local and systemic.

Young, female, alloxan-diabetic rats (40 animals) were subjected for 65 days to daily injections of 3-5 units of protamine zinc insulin into the fat pad of one groin, with control injections of saline into the other groin. The insulin-injected fat pad showed an increase in weight averaging 55 per cent, an increase in total ether-extractable material averaging 66 per cent and a definite increase in the size of individual fat cells. The ability to deposit glycogen under the influence of insulin was not consistently altered at the site of the prolonged injections.

From these data it is concluded that there is evidence of a local metabolic action of insulin in adipose tissue. This action might be attributed to the increased production of metabolically available carbohydrate-glycogentherefore to a possible favoring action on the conversion of carbohydrate to fat in the adipose tissue itself. Considerable evidence for the existence of extrahepatic lipogenesis is available in the literature.

The Relation of Effective Pulmonary Blood Flow to Total Pulmonary Blood Flow in Normal Man and in Patients with Various Types of Chronic Pulmonary Disease. Richard L. Riley, Robert Austrian, KenNeth $W$. Donald and ANDre Cournand (Introduced by Dickinson W. Richards, Jr.), New York, N. Y.

With knowledge of the composition of inspired and expired air, the respiratory quotient and the tensions of $\mathrm{O}_{2}$ and $\mathrm{CO}_{2}$ in systemic arterial blood, it is possible to determine indirectly the composition of alveolar air and of pulmonary capillary blood that would obtain if homogeneous equilibrium existed between blood and gas phases throughout the lung. When the composition of mixed venous blood is also determined, one can compute the proportions of mixed venous blood and of effectively oxygenated pulmonary capillary blood that, when mixed, would yield blood of the composition found in the systemic arteries. Effective pulmonary blood flow is defined as the product of total cardiac output times the calculated proportion of effectively oxygenated pulmonary capillary blood.

In normal individuals, effective pulmonary blood flow averaged 95.8 per cent of total pulmonary flow. In patients with pulmonary suppuration and in those with pul- 
monary fibrosis, the effective fraction of total flow averaged 92 per cent and 88 per cent respectively. In a group of patients with severe emphysema, the average effective fraction of flow was 69 per cent, a value only slightly higher than that found in a proven case of pulmonary arteriovenous fistula.

The Summation of Penicillin and Streptomycin Activity in Vitro and in the Treatment of Subacute Bacterial Endocarditis. WIILIAM C. RobBINS (Introduced by David P. Barr), New York, N. Y.

Despite the therapeutic effectiveness of penicillin in subacute bacterial endocarditis, ultimate failure occurs in approximately 10 per cent of all cases because of inadequate suppression of the causative organism even by massive doses. Moreover, the use of streptomycin in these cases, most of which are caused by enterococci, has effected little improvement in the results.

An investigation has been made of the combined activity of penicillin and streptomycin in vitro with particular reference to the quantitative relationships of their combined action on strains of enterococci isolated from patients with endocarditis. It has been observed that penicillin and streptomycin act as coadjuvants in inhibiting growth of enterococci. Total summation of partial effects has been found to occur throughout a wide range of concentrations of each constituent drug. For example, complete inhibiton of growth is achieved with a mixture in which each drug is present in a concentration only half that of its own inhibitory concentration.

Five patients with persistent enterococcal bacteremia, four of whom had endocarditis, have received penicillin and streptomycin concurrently in doses of moderate size. Two patients received the combined therapy terminally, but three completed a four to eight weeks course of this therapy.

In all three patients bacteremia was promptly reversed and the immediate striking clinical improvement was sustained. The apparently complete recoveries have been maintained for six to fifteen months. Two additional patients are now under treatment.

The uniformly good results in these patients has been in striking contrast to the previous general experience with the use of either drug alone, and suggests the operation in vivo of a summative effect comparable to that observed in vitro.

The Effects of Oral Therapy with Cobaltous Chloride in the Blood. JOSEPH C. RoBinson (by invitation), RoBERT M. KarK, (by invitation) MARY Maloney, Frederick Sargent, II, and George W. James, Chicago, III., and Richmond, Va.

Although cobalt is an effective erythropoietic agent in cobalt deficient and healthy animals, its effects on the blood of healthy adults and of patients suffering with various types of hematologic disturbances have not been clearly defined. When administered orally cobaltous chloride (20 to $240 \mathrm{mgs}$. per day) induced anorexia in some patients but no other toxic symptoms were observed.
With the above dosage schedule, increased erythropoiesis was observed in 15 patients ill with chronic sepsis, in two patients suffering with skeletal neoplastic metastases, and in one patient with an unexplained longstanding refractory anemia. No erythropoietic response was noted in patients suffering with pernicious anemia when they were fed cobaltous chloride either alone or incubated with normal gastric juice. The drug was without effect in a patient ill with nutritional macrocytic anemia.

The blood of 15 young adults ill with chronic suppurative infection, when compared with the blood of 10 bedridden controls, showed abnormal iron and copper concentrations in plasma, a decreased red cell mass and a reduced total circulating hemoglobin. Following treatment with cobaltous chloride reticulocyte responses were observed in each patient. The maximal reticulocyte percentages were usually noted between the sixth and tenth day and did not exceed 5 percent in any patient. Thereafter, a steady increase was noted in red blood cell counts, in hematocrit values and in hemoglobin levels. The plasma volume changed but slightly whereas the circulating red cell mass and the total circulating hemoglobin increased about 30 percent.

In an attempt to explain the mechanism of cobalt activity on the basis of impaired cellular respiration, the blood of patients was examined spectrophotometrically. No abnormal concentrations of methemoglobin were observed.

Tyrosine Metabolism in Human Scurvy. WALTER F. Rogers, JR., and Frank Gardner (Introduced by Robert F. Pitts), Syracuse, N. Y.

It has previously been reported that scorbutic guinea pigs, and premature infants fed high protein diets, have a defect in their metabolism of tyrosine which is corrected by vitamin $C$. These findings have prompted the study of tyrosine metabolism in 4 individuals with scurvy and in 4 normal persons.

After suitable control periods all individuals, with the exception of one scorbutic, received 20 grams of tyrosine by mouth daily for periods varying from five to thirty-one days. The patients with scurvy, while on a vitamin $C$ free diet, and under this load of tyrosine, excreted large amounts of "tyrosyl" derivatives, and the power of their urine to reduce phosphomolybdic acid increased markedly, presumably due to the presence of p-hydroxyphenylpyruvic acid. Compared to normal individuals receiving 20 grams of tyrosine per day, the scorbutics excreted from seven to fifteen times the quantity of "tyrosyl" derivatives, and the power of the urine of scorbutics to reduce phosphomolybdic acid was twenty to thirty times that of normals, the urine of the latter showing no increased power to reduce phosphomoylbdic acid during the administration of tyrosine. The administration of vitamin $\mathrm{C}$ to scorbutics during tyrosine loads completely corrected the metabolic abnormality in from twenty-four to forty-eight hours.

The administration of these large amounts of tyrosine 
appeared to exacerbate the clinical condition of the individuals with scurvy.

Variations in the Electrophoretic Pattern of Synovial Fluid in Articular Disease. MARIAN W. Ropes and (by invitation) Dorothy Kaufman and Gertrude E. Perlmann, Boston, Mass.

As reported previously, comparison of the electrophoretic patterns of synovial fluid and serum in rheumatoid arthritis supports the hypothesis that there is increased permeability to protein of the tissues interposed between the blood and the joint space. In some of the fluids the distribution of proteins followed closely that found in the corresponding serum. In other cases there was a marked elevation of gamma-globulin in the synovial fluid despite a relatively low concentration of this component in the serum.

Study of electrophoretic patterns of synovial fluids from traumatic arthritis suggests that there is differential permeability to individual protein fractions. Albumin was always higher in the fluid than in the serum, alpha-1 and beta-globulins essentially the same, and alpha-2 and gamma-globulins lower in the fluid.

Further study of rheumatoid fluids has indicated some correlation between the electrophoretic pattern of the synovial fluid, the clinical features of the involved joints and the other characteristics of the fluid. In patients with early or mild involvement of the joint studied, the electrophoretic pattern usually resembled that of traumatic fluids, with albumin higher in the fluid than in the serum and gamma-globulin in equal or lower concentration in the fluid. In cases with longer duration or greater severity of inflammation in the joint studied, the electrophoretic pattern tended to be different. The albumin concentration was equal in the fluid and serum and the gamma-globulin higher in the fluid.

The electrophoretic pattern described in the previous report, with marked elevation of the gamma-globulin in the fluid above that in the serum, was found in three cases showing atypical clinical pictures. In these patients, large effusions had been present for one to eight years, but there was relatively little objective evidence of joint damage and other studies of the synovial fluid indicated relatively mild inflammation.

The Inhibitory Effect of Secretions of the Human Respiratory Tract on Influenza Virus. HARRY M. ROSE, New York, N. Y.

Aqueous preparations of sputum from individuals with a number of diseases accompanied by cough and expectoration contain variable amounts of a substance which will inhibit the agglutination of erythrocytes by influenza virus type A (PR8) and B (Lee). This inhibitor is not specific antibody and is unrelated to the mucin content of sputum, as determined by the ACRA test, or to the level of antibodies in the blood. It acts directly on the virus and not by altering the erythrocyte.

The inhibitor is moderately heat stable but is destroyed in 30 minutes at $100^{\circ} \mathrm{C}$. Its activity is essentially un- changed over a $\mathrm{pH}$ range from 4.0 to 8.0 . It may be partially purified and concentrated by treating sputum with chloroform, followed by precipitation with ethanol at low temperature. The partially purified material is more stable on storage than the original sputum.

The titer of inhibitor is higher against influenza virus heated to $56^{\circ} \mathrm{C}$. for 30 minutes than it is against the same number of agglutinating units of living virus. This effect is more pronounced with Lee than with PR8 virus.

The inhibitor is destroyed by exposure to living PR8 virus but not by exposure to heated virus. Its activity is also moderately reduced by treatment with the R.D.E. of Vibrio cholerae.

Mixtures of inhibitor and PR8 virus which fail to agglutinate erythrocytes when first prepared show a progressive rise in hemagglutinin titer on standing at $37^{\circ} \mathrm{C}$. This is interpreted to indicate the release of virus from an inhibitor-virus complex. Such eluted virus may or may not show a reduction in its infectivity for chick embryos.

Preparations of the inhibitor may neutralize up to 1,000 minimal infectious doses of PR8 virus in mice. Previous treatment of the inhibitor with living virus reduces its neutralizing potency.

Effect of Urethane (Ethyl Carbamate) in Multiple Myeloma. R. Wayne Rundles, M. L. Dillon, Edith S. Dimlon and John Armstrong (Introduced by David T. Smith), Durham, N. C.

Eleven patients with multiple myeloma have been treated with urethane. One could not tolerate the chemical and three died in 1-5 weeks. Seven patients were given 120-240 grams of urethane in 8-10 weeks and the course of their disease studied over periods up to 16 months. Striking clinical benefits were observed, including subsidence of fever, relief of skeletal pain, and gain in weight and strength. Incapacitated individuals were able to resume normal activities.

Measurable improvement related to all aspects of the disease. Abnormal plasma or "myeloma" cells became fewer or disappeared from the bone marrow and showed morphologic changes indicative of retarded or arrested growth. Four individuals had severe anemia. After urethane therapy abnormal cells disappeared from the circulating blood. The hemoglobin and red cell values improved, in three cases to normal levels. There was no progression in the osteolytic skeletal lesions as judged by serial roentgenograms. Bony recalcification was demonstrable in individuals with extensive skeletal disease after 4-6 months. Serum calcium levels when elevated fell to normal. The serum proteins were studied by electrophoretic methods in five patients. After two months of urethane therapy the abnormal globulin components decreased conspicuously or virtually disappeared. The amount of Bence Jones protein in the urine decreased. There was no association between the type of serum protein abnormality or the amount of proteinuria and renal function impairment. Nitrogen retention did not develop after urethane therapy. 
Incomplete remissions of the disease have been observed as well as relapses $2-6$ months after discontinuing urethane. Successive courses of urethane were given with good results. Despite these reservations the therapeutic benefit of urethane in multiple myeloma exceeds that of any other agent in use at this time.

\section{Fatal B Virus Encephalomyelitis in a Physician Working} with Monkeys. AlBerT B. SABIN, Cincinnati, Ohio.

This is to report for the second time in the past 16 years that a physician, aged 25 , engaged in experimental work with rhesus monkeys, died from an infection of the central nervous system due to B virus, which occurs in monkeys much like herpes simplex virus occurs in man. The first case, a fatal acute ascending myelitis, from which the B virus was isolated and identified, occurred in 1932 in a young physician who was bitten by a monkey. In the present case there was no bite, but the infection apparently resulted from contamination by monkey saliva of a minor cut on the right index finger. This was followed by an insignificant lesion at the site of the cut, lymphangitis, lymphadenitis, and within a few days thereafter by a febrile illness during which the following significant neurological manifestations were observed in the order mentioned: crops of small herpetic-like lesions on the inner aspect of the right forearm, arm, axilla and anterior chest wall associated with soreness and burning sensation, dysphagia, increasing mental confusion, absent deep tendon reflexes in lower extremities, inability to expel enema, urinary retention, bilateral nystagmus, irregular respiration and finally cessation of respiration. The most severe lesions were found in the gray matter of the posterior and lateral horns of the spinal cord, in adjacent areas of the white matter, in the regional spinal ganglia, and medulla. An infectious agent possessing the properties previously described for B virus was recovered from the affected right axillary lymph node and central nervous system, by inoculation of rabbits. Mice proved to be relatively refractory and passage was not possible in them. No virus was found in the patient's cerebrospinal fluid, liver and spleen. The virus recovered from the patient was neutralized by a specific $B$ virus antiserum.

Unusual Serological Findings in a Group of Cases of Pneumococcal Pneumonia Associated with the Presence of Influenza Virus in the Sputum. JonAs E. SALK and (by invitation) MARY P. Lynch, Pittsburgh, $\mathrm{Pa}$.

Between October 1948 and February 1949, when influenza was not occurring epidemically in the area in which these observations were made, 73 cases of pneumonia were studied, 5 of which yielded influenza virus upon inoculation of sputum into developing chick embryos. The viruses isolated in these cases, and from an additional case of influenza-like illness, were all found to be related antigenically to the A-prime group of influenza virus Type A. Pneumococcus Type I was found in the sputum of 2 cases, Type II in two other cases, and Type XVI in the sputum of the fifth case. Bacteremia was present in three instances; the patients with Type I pneumonia were found to have 1,260 and 306 colonies per cc. of blood, respectively, and one with Type I pneumonia had 11 colonies per cc.

It is of particular interest that the presence of the virus in the sputum of these patients would not have been surmised from the results of the serological studies employing the hemagglutination-inhibition test and the complement-fixation test. Even with homologous antigens, these tests failed to reveal any increase in antibody in convalescence. However, definite evidence of antibody increase was observed in one case, and suggestive evidence in another, by a method involving virus neutralization tested in ovo.

Although a satisfactory explanation for the vagaries in the serological findings is not immediately apparent, several plausible possibilities exist. These are at present under investigation and will be presented and discussed.

The Effects of Postural Changes, Stellate Ganglion Block, and Anemia on the Cerebral Circulation. PERITz Scheinberg (Introduced by W. M. Nicholson), Durham, N. C.

Many mechanisms influencing cerebral circulation can now be reinvestigated semi-quantitatively by the nitrous oxide technique for cerebral blood flow, devised by Kety and Schmidt. This technique was modified by drawing continuous 10-minute samples of arterial and internal jugular blood, thus allowing the body to integrate the mean nitrous oxide contents rather than calculating them mathematically from 5 separate arterial and venous samples.

Effective cerebral arterial pressure was reduced by tilting to an angle of $65^{\circ}$ from the horizontal. Unilateral release of sympathetic tone was accomplished by procaine block of the stellate ganglion. Patients with anemia had: (1) chronic blood loss of varying durations; (2) pernicious anemia.

In 13 normals the average reduction in effective mean arterial pressure on tilting was 34 per cent. Average decrease in cerebral blood flow was 21 per cent, with average increase in cerebral arterio-venous $\mathrm{O}_{2}$ difference of 23 per cent. Cerebral $\mathrm{O}_{2}$ utilization (cerebral blood flow $\times \mathrm{A}-\mathrm{V} \mathrm{O}_{2}$ ) did not change. Average cerebrovascular resistance (mean arterial pressure $\div$ cerebral blood flow) fell 16 per cent. Average fall in internal jugular venous pressure was $60 \mathrm{~mm} . \mathrm{H}_{2} \mathrm{O}$.

Stellate blocks were done on 16 subjects. These fell into 3 groups: (1) normal young individuals; (2) elderly patients with diffuse vascular disease; and (3) elderly patients with acute cerebral vascular accidents. There was no significant change in cerebral blood flow, $\mathrm{O}_{2}$ utilization, or cerebro-vascular resistance following stellate blocks in any of the groups.

In 7 patients with anemia from chronic blood loss cerebral blood flows were increased greatly over the normal; the cerebral $\mathrm{A}-\mathrm{V} \mathrm{O}_{2}$ differences were proportionately reduced, giving normal values for cerebral $\mathrm{O}_{2}$ utilization. The cerebro-vascular resistance was markedly diminished in all. 
In 5 patients with pernicious anemia the cerebral oxygen utilization was strikingly decreased. In one the value was normal.

Renal Insufficiency Caused by Overhydration or Depression of Sodium Chloride: The "Loze-Salt Syndrome." Henry A. Schroeder, St. Louis, Mo.

Under certain circumstances, especially when there is slight impairment of renal function due to the aging process, hypertension or congestive circulatory failure, oliguria leading to renal excretory insufficiency may develop rapidly. When the intake of sodium chloride is restricted and the intake of water is not, or when depression of body salt and not of water is induced, the result is nitrogen retention, dilution of extracellular electrolytes, overhydration and probably hydration of cells. Death occurs from uremia. This condition was encountered in over 30 cases, of which 10 died, under the following circumstances: (a) In congestive failure after the enthusiastic use of mercurial diuretics, (b) In congestive failure with slight renal damage when the intake of fluids was rapidly elevated, (c) In arterial hypertension with moderate renal insufficiency when the intake of salt was severely restricted, (d) Post-operatively, in older individuals, when the concentration of plasma electrolytes was diminished by too vigorous use of glucose in water, (e) When excessive electrolyte losses from surgical drainage (bile, etc.) had occurred. While the electrolyte pattern resembled that seen in anuria from other causes when fluids and not salt were given, renal function could be often restored by increasing the concentration of the electrolytes in extracellular fluids to normal levels. The intravenous injection of hypertonic saline solution (5-6 per cent), in an amount sufficient to replace the calculated deficit in extracellular fluids, was the method of choice. When this was used, diuresis often became established and renal function returned toward normal. No adverse effects from the use of hypertonic salt were observed or expected when electrolyte levels were depressed even in the presence of general anasarca, pulmonary congestion, and elevation of the venous pressure. The early recognition of oliguria due to overhydration or salt depletion is necessary for therapy to be effective. It is probable that many cases of so-called "post-operative," "reflex" and "essential" anuria are the result of overhydration. Shock was not necessary for the situation to develop.

Some Biochemical Effects of Radiation. SAMUEL Schwartz (Introduced by Jay C. Davis), Minneapolis, Minn.

The effect of relatively large amounts of ionizing radiations on several biochemical systems has been studied in human subjects and in experimental animals (dogs, rabbits, and rats). The following changes have been observed:

(1) There is a marked rise in excretion of urinary corticosteroids in human subjects.

(2) There is a significant increase in bile pigment excretion indicating that radiation anemia is in part hemolytic in nature.
(3) There is a marked fall in the urinary and fecal excretion of coproporphyrin.

(4) Evidence of liver dysfunction is found shortly after the irradiation and especially during the several days preceding death.

(5) Marked increases are often found in the urinary urorosein reaction in humans. Another tryptophane derivative, kynurenic acid, is excreted in increased amounts by dogs.

(6) Increases are often observed in the adenosine triphosphate fraction of the circulating white blood cells. (This may be noted with relatively small amounts of radiation, but is not uniform.)

Excretion of Sodium Following Sulfanilamide Administration in Congestive Heart Failure. William B. Schwartz (Introduced by Samuel A. Levine), Boston, Mass.

It has been the hypothesis in this study that inhibition of carbonic anhydrase in the renal tubules should result in an increased excretion of sodium. Since the acidification of the urine appears to be effected by hydrogen ions within the renal tubular cells being exchanged for sodium ions in the glomerular filtrate, it was postulated that diminished tubular excretion of hydrogen should lead to a decreased reabsorption of sodium. For this reason sulfanilamide, a specific inhibitor of carbonic anhydrase, has been administered in an effort to lower the rate of formation of carbonic acid in the renal tubules and thus reduce the source of hydrogen ions available for excretion. Seven subjects in refractory congestive heart failure on constant fluid and food intakes have received sulfanilamide in doses of three to six grams per day for periods of two to eight days. Increased excretions of sodium averaging three to four times the control values have resulted in each case. There was also, in most instances, a prompt increase in potassium and water excretion and loss of weight proportional to the increased sodium excretion. At the same time there was a fall in the carbon dioxide combining power of the serum and an elevation of the serum chloride level. Significant improvement in the signs and symptoms of congestive heart failure occurred in five out of seven patients. Other compounds with unsubstituted sulfonamide groups which are inhibitors of carbonic anhydrase are under investigation in an effort to find a substance less toxic than sulfanilamide.

The Effect of Endocrine Therapy on Hormonal Excrstion in Patients with Carcinoma of the Breast with Extension. Albert Segaloff and (by invitation) Richard L. Coppedge, New Orleans, La.

Studies of various hormonal excretions have been done in patients with metastatic carcinoma of the breast. Many of the patients showed levels of gonadotrophic hormone excretion much lower than one would expect from their age and endocrine status. This is believed due to their advanced cachectic state as were the low ketosteroid values observed in many of these same patients. In the patients in whom gonadotrophic hormones were. sufficiently high before the initiation of therapy, both estrogens and andro- 
gens produced the expected decrease of gonadotrophic hormone in the urine. Most of the patients rece 1 ving testosterone propionate showed the expected rise in ketosteroid excretion and androgen excretion. However, two of the patients with previously low ketosteroid values had no increase in ketosteroid excretion.

In 3 of the 6 patients in whom follow-up cortins were obtained, there was a very distinct rise in the excretion of cortin while on testosterone therapy. In the one patient under estrogen therapy in whom this has been studied, there was no change in the excretion of cortin in the urine despite a favorable clinical response to the estrogen.

In 3 of the 4 patients in whom the urinary excretion of lactogenic hormone has been studied before and during therapy with testosterone, there was a distinct rise in prolactin excretion during the therapy with testosterone. In one of these 3 patients, estrogen produced no further change.

There has been no very striking correlation between the clinical course observed in any of these patients and the hormonal changes in the urine. However, the patients obtaining the best clinical responses to testosterone were the patients who showed the increases in cortin excretion.

The Metabolism and Excretion of Elcctrolytes and Glucose in Diabetic Acidosis. Donald W. Seldin and Robert Tarail (Introduced by David M. Kydd), New Haven, Conn.

Fifteen patients, studied during the early stages of diabetic acidosis, received repeated moderate or massive injections of glucose. Utilization and storage of carbohydrate probably occurred within the first two to three hours of treatment, since the injected glucose could not be completely recovered in the extracellular fluid or urine. The large increment of administered glucose which was excreted after massive injections resulted in a striking increase in urine flow despite dehydration. Potassium was not swept into the urine by the osmotic action of glucose within the renal tubule, since the excretion of the ion was highest initially and fell progressively notwithstanding marked increases in glycosuria. The excretion of sodium and chloride, in contrast, appeared to be augmented by infusions of glucose, although this response was modified by adaptive reactions such as conservation of sodium in the maintenance of acid-base equilibrium.

The serum concentrations and urinary losses of potassium and phosphorus were highest initially, and fell progressively during treatment. The high serum concentrations resulted from the failure of the initially accelerated excretion to keep pace with the effects of rapid cellular release of the ions and a contracting extracellular volume. After treatment was instituted, however, the discharge of potassium and phosphorus from cells was progressively retarded. Indeed, positive intracellular balances of phosphorus were observed in six of seven patients and of potassium in two of eight patients. The slow cellular release of the ions (possibly due to increased utilization of carbohydrate and diminished catabolism of protein), in conjunction with continuing losses in the urine and expan- sion of extracellular volume were responsible for the fall in the serum concentrations.

The Effect of Lumbodorsal Sympathectomy upon Insulin Tolerance in Man. F. A. Simeone (Introduced by Oliver Cope), Boston, Mass.

The insulin tolerance of non-diabetic hypertensive patients selected for lumbodorsal sympathectomy did not differ from that of non-diabetic non-hypertensive patients of the same age group. In the cat, dog, and rabbit, denervation of the adrenal glands renders the animals extraordinarily sensitive to insulin. In a previous report, it was demonstrated that, contrary to expectation, the insulin tolerance of patients who had had lumbodorsal sympathectomy was increased during the immediate postoperative period. This finding was interpreted as a manifestation of the adaptation syndrome.

Twenty-six patients have been studied approximately one year after lumbodorsal sympathectomy and the insulin tolerance curves have been compared with those before operation and with those immediately after operation in the same patients. The sensitivity to insulin a year after operation was significantly greater than it was before operation or soon after operation.

In three of the twenty-six patients, the increased sensitivity to insulin a year after operation was especially striking, the blood sugar concentration remaining 20 per cent below fasting as long as 180 minutes after the injection of insulin. One of these three had a convulsion after insulin, 0.08 units per kilogram of body weight intravenously). No other patient had a convulsion before or after operation.

In summary, lumbodorsal sympathectomy is attended by increased sensitivity to insulin demonstrable after a preliminary period of increased tolerance, a manifestation of the adaptation syndrome. In the majority of cases, however, the change in sensitivity is not great enough to be a useful test of adrenal denervation for the individual patient.

The Site of Action of Methyltestosterone in the Production of Creatinuria. ETHAN A. H. Sims (Introduced by Arthur J. Geiger), New Haven, Conn.

It is generally accepted that methyltestosterone ingestion produces increased creatine synthesis, which is followed by creatinuria when the capacity to store creatine is exceeded. The initial rise in guanidoacetic acid (GAA) serum levels and excretion indicates accelerated GAA synthesis. It has not, however, been demonstrated that creatinuria may be produced solely by increased GAA synthesis. Failure to recover quantitatively exogenous creatine or GAA in balance studies, including a recent study in which the subject's tissue creatinue was labeled with $N-15$, suggested that the methylation of GAA is retarded by excess creatine. If this were so, it would be necessary to postulate that methyltestosterone accelerates the methylation as well as the synthesis of GAA.

GAA tolerance tests made after the ingestion of $25 \mathrm{gm}$. of creatine gave results inconsistent with a retarded con- 
version of GAA to creatine under these conditions. To determine whether GAA is in fact methylated when the tissues are loaded with creatine, a normal subject was given $2.5 \mathrm{gm}$. of GAA daily in 16 hourly portions after the body had been saturated by prolonged creatine ingestion. A creatinuria averaging $0.48 \mathrm{gm}$. was seen during the following 21 days. The creatinine excretion did not fall during this period, suggesting that the urinary creatine did not originate from body creatine. All creatine values were corrected for glycocyamidine. GAA excretion averaged $0.55 \mathrm{gm}$. per day during the period of creatinuria. No evidence suggesting retardation of methylation of GAA was found under these conditions. It was thus possible to simulate the creatinuria from methyltestosterone by administering GAA in physiological amounts. This suggests that the direct action of the steroid on creatine metabolism may be solely that of accelerating GAA synthesis.

The Dynamics of the Cellular Response of Lymph Nodes to Acute Bacterial Infection. Ralph O. Smith (by invitation) and W. BARRY Wood, JR., St. Louis, Mo.

In order to define more precisely the role of iymphatic tissue in the cellular mechanism of antibacterial defense, a study was made of the pathogenesis of acute popliteal lymphadenitis produced in rats by inoculation of the footpad with Type I pneumococcus. Early in the course of the infection the bacteria reaching the popliteal node via lymphatics caused a rapid exudation of polymorphonuclear leucocytes within the nodal sinuses. The granulocytes which accumulated in the intermediary sinuses were found to come primarily from capillary blood vessels of the node, whereas the majority of inflammatory cells in the subcapsular sinus arose from the primary focus of infection in the foot. The granulocytes in the sinuses, carried toward the hilus by the continuous flow of lymph, formed a "log jam" of cells through which invading bacteria were forced to pass. The hilar accumulation of cells greatly increased the filtration efficiency of the node, and pneumococci caught among the phagocytes were promptly ingested. Mast cells lining the sinuses were noted to discharge their heparin containing granules into the nodal lymph. Whether this mast cell reaction accounts for the failure of lymph within the node to clot or whether the released heparin plays some other role in antibacterial defense is now being investigated. It is concluded that lymph nodes, often regarded as passive filters in the lymph stream, in reality react promptly to bacterial invasion and provide an active cellular barrier to bacterial invasion of the blood stream.

Liver Function Studies in Amebiasis. W. A. Sodeman and (by invitation) A. A. Doerner, New Orleans, La.

Liver function tests (cephalin flocculation, thymol turbidity, quantitative blood bilirubin, bromsulfalein) were done in a group of 51 patients with colonic amebiasis in the absence of dysentery, clinical evidences of malnutrition and past history of hepatic disease. They fell into four groups: (1) Acute amebic abscess, (2) Acute amebic hepatitis without demonstrable abscess, (3) Colonic amebiasis with tender hepatomegaly but without systemic response, and (4) Colonic amebiasis without demonstrable hepatomegaly. Tests were done before therapy was instituted and, where possible with abnormalities, after therapy was completed.

Approximately 50 per cent of those in groups 1 and 2 showed evidence of hepatic damage, chiefly with the cephalin flocculation test and with minor elevations of the blood bilirubin. In 10 per cent there was clinical jaundice. In group 3 the tests were positive in about 20 per cent and, like group 4, were spotty and irregular.

These studies indicate that (1) in the absence of a febrile response, the variability of results was not striking. (2) Although diffuse damage occurs in the liver from amebiasis and is reflected in liver function tests, the process at times may reach advanced stages with no more evidence of involvement, by the technics used, than in those with subclinical involvement. (3) The number of such discrepancies between the clinical findings and the liver function tests used is in contrast with that seen in some other acute conditions of the liver and may be helpful at times in differential diagnosis. (4) Moderate degrees of hepatomegaly, unexplained and without confirmatory positive tests of liver function, are at times the chief physical findings of amebiasis and should lead to search of the colon for this disease. (5) Amebiasis may, at times, without clinical signs of hepatic involvement give evidence of hepatic involvement with the liver function tests used.

Coronary Blood Flow and Cardiac Oxygen Consumption in Unanesthetized Dogs. F. C. Spencer, S. R. Powers, D. L. Merrill and R. J. Bing (Introduced by Cowles Andrus), Baltimore, Md.

In a large series of experiments on trained, unanesthetized dogs, the coronary blood flow through the left ventricle has been determined by the combination of coronary sinus catheterization and the nitrous oxide method. A catheter was inserted irito the coronary sinus through the external jugular or cephalic vein; arterial blood was collected from the femoral artery. Following a ten-minute saturation with 15 per cent nitrogen oxide, samples were collected over a desaturation period of eight minutes. Mixed venous blood was collected simultaneously through a separate catheter in the pulmonary artery. Left ventricular weight was calculated from the tables of Herrmann.

The left ventricular flow averaged $151 \mathrm{cc} . / 100$ grams/ minute, the coronary arterio-venous oxygen difference 12.8 vol. per cent, and the oxygen consumption of left ventricular muscle $19.5 \mathrm{cc} . / 100 \mathrm{gram} /$ minute. The oxygen consumption of the whole left ventricle averaged $11.3 \mathrm{cc}$. of oxygen / minute. The left ventricular efficiency

$$
\left[\frac{\text { left ventricular work (kgm. meters) }}{\text { energy cost of whole left ventricle (kgm. meters) }}\right] \text {, }
$$

calculated from an average cardiac output of 4,480 cc./ minutes and a mean aortic pressure of $119 \mathrm{~mm}$. $\mathrm{Hg}$ was found to be 30.7 per cent.

It may be concluded that in the unanesthetized dog both left ventricular flow and oxygen consumption per unit 
weight are twice as high as in the anesthetized dog (Edkenhoff $e t a l$ ). Accordingly the left ventricle efficiency is within the range observed in normal man.

Metabolic Effects of Synthetic Compound E (17-Hydroxy-11-Dehydrocorticosterone) in 2 Patients with Addison's Disease and in 1 with Coexisting Addison's Disease and Diabetes Mellitus. Randall G. Sprague and (by invitation) Marschelle H. Power, Harold L. Mason and Harley E. Cluxton, Rochester, Minn.

Response to the administration of synthetic compound $\mathrm{E}$ (17-hydroxy-11-dehydrocorticosterone) was studied in 2 female patients with Addison's disease and in 1 with both Addison's disease and diabetes mellitus during treatment with 11-desoxycorticosterone. Compound $\mathrm{E}$ acetate was administered intramuscularly as an aqueous suspension of finely ground crystals in doses of 50 and $100 \mathrm{mg}$. daily, respectively, to the 2 patients with Addison's disease, and in doses of $30 \mathrm{mg}$. to the patient with both Addison's disease and diabetes. In another study, the last patient received $25 \mathrm{mg}$. of free compound $\mathrm{E}$ daily.

Under the conditions of these studies, compound $\mathrm{E}$ produced no significant change in the urinary excretion of sodium, potassium or chloride. Likewise, minor changes in the excretion of nitrogen, calcium and phosphorus were probably not significant.

During treatment with compound $\mathrm{E}$, both patients with Addison's disease were able to fast without decline of the level of the blood sugar. A minor reduction in sensitivity to insulin, and higher levels of the blood sugar in the course of glucose tolerance tests were also observed. In the case of coexisting Addison's disease and diabetes mellitus, carbohydrate effects of the hormone were more easily demonstrated. During treatment with compound E, there was a marked increase in urinary glucose, in spite of the daily administration of 4 or more times the amount of insulin employed in the control periods.

During and following periods of administration of compound $\mathrm{E}$ acetate, the urinary 17 -ketosteroids and corticosteroids increased above the control levels. In 1 patient, the excretion of these substances remained elevated for a period of four weeks after the administration of compound $\mathrm{E}$ acetate was discontinued, and there was evidence of persistence of its physiologic effects during this time, suggesting that the steroid was absorbed slowly from the sites of injection.

The results demonstrate the predominant carbohydrate activity of compound $\mathrm{E}$. The hormone was excreted in part as a $17-$ ketosteroid.

Cardiovascular-renal Dynamics and Electrolyte Excretion in Panhypopituitarism. J. Stamler, A. P. Fishman and R. Rosenman (Introduced by L. N. Katz), Chicago, Ill.

Increased appreciation that hormonal factors influencing mineral exchange may play a role in the initiation and persistence of congestive heart failure led us to study the cardiovascular-renal dynamic changes in various endocrine disorders usually associated with electrolyte disturbances. This report presents the initial data in this investigation.
In two cases of panhypopituitarism, with B.M.R.'s of -48 and -23 respectively, studies of the cardiovascularrenal dynamic changes by cardiac catheterization and renal clearance techniques revealed the following changes: decreased cardiac output, cardiac index, circulation rate, renal blood flow, renal index (renal blood flow/cardiac output), glomerular filtration rate, and blood volume, and normal thiocyanate space and venous pressure.

Patient A presented symptoms suggestive of adrenal insufficiency (plasma $\mathrm{Cl} 90, \mathrm{Na} 134 \mathrm{~m}$. eq./L) ; patient $\mathrm{B}$ had no such findings (plasma $\mathrm{Cl} 102, \mathrm{Na} 144 \mathrm{~m}$. eq./L). With distal tubular function calculated on the basis $(\mathrm{H}$. W. Smith) that there is an obligatory isotonic proximal tubular reabsorption of 85 per cent of the glomerular filtrate, renal clearance data reveal the following: In patient A, with plasma $\mathrm{Cl}$ concentration of $89-91 \mathrm{~m}$. eq./L, 6.7-7.4 per cent of the $\mathrm{Cl}$ filtered (GFR: $47 \mathrm{cc} . / \mathrm{min}$.) is excreted; the overall tubular reabsorate has a $\mathrm{Cl}$ concentration of $86-87 \mathrm{~m}$. eq./L; distal tubular $\mathrm{Cl}$ reabsorption is at the rate of $0.3-0.4 \mathrm{~m}$. eq. $/ \mathrm{min}$; the distal reabsorbate has a $\mathrm{Cl}$ concentration of $58-62 \mathrm{~m}$. eq./L. In patient $\mathrm{B}$, despite a higher plasma $\mathrm{Cl}$ concentration (94-104 m. eq. /L) and despite a higher GFR (58 cc./min.) only 2.0-4.2 per cent of the $\mathrm{Cl}$ filtered is excreted; the overail tubular reabsorbate has a higher $\mathrm{Cl}$ concentration (102-114 m. eq. /L) ; distal tubular $\mathrm{Cl}$ reabsorption occurs at a greater rate $(0.7-0.8 \mathrm{~m}$. eq. $/ \mathrm{min}$.) ; by further contrast, the distal $\mathrm{Cl}$ reabsorbate is hypertonic $(267-485 \mathrm{~m}$. eq. $/ \mathrm{L})$, and this cannot be accounted for by impaired $\mathrm{H}_{2} \mathrm{O}$ reabsorption. Thus, the inadequacy of distal electrolyte absorption in patient $\mathrm{A}$, even at low plasma $\mathrm{Cl}$ levels, contrasts markedly with that of patient $\mathrm{B}$ and accounts for the hypochloremia and hyponatremia in the former.

It is suggested that patient $A$, unlike patient $B$, is one of the more unusual cases of panhypopituitarism suffering from a secondary adrenal insufficiency involving the mineralocorticoids. In such a patient both the decreased demands upon the heart (low B.M.R.) and the decreased tubular reabsorption of electrolytes secondary to adrenal insufficiency mitigate against the development of congestive heart failure and edema, despite myocardial damage and low glomerular filtration rate (decreased presentation of $\mathrm{Na}$ to the renal tubules).

The Effect of the Iodide Ion on Thyroid Hormonc Formation in Man. Malcolm M. Stanley (Introduced by E. B. Astwood), Boston, Mass.

The amount of iodine entering the thyroid normally provides an index of hormone formation. Stable thyroid iodine $\left(\mathrm{I}^{127}\right)$ accumulation can be estimated with radioactive $\mathrm{I}^{131}$ provided (1) That thyroid $\mathrm{I}^{131}$ can be measured and (2) That the specific activity of the collected $\mathrm{I}^{131}$ can be computed. $\mathrm{I}^{127}=\mathrm{I}^{131} / \mathrm{S}$.A.

Nearly simultaneously, serially, urine $I^{127}$ and $I^{131}$, serum $\mathrm{I}^{131}$ and thyroid $\mathrm{I}^{131}$ were determined, the last by counting at 35 centimeters from the gland. The kidneys excreted these isotopes in the urine in the same ratio in which they appeared in the serum: $\mathrm{SI}^{127} / \mathrm{SI}^{131}=\mathrm{UI}^{127} / \mathrm{UI}^{131}$. The renal and thyroid arterial blood iodide contents were identical ; the urinary $\mathrm{I}^{131}: \mathrm{I}^{12 \pi}$ ratio reflected the specific activ- 
ity of the thyroid radioiodide accumulated during the period. Serum iodide $\left(\mathrm{I}^{127}\right)=\mathrm{SI}^{131} \times \mathrm{UI}^{127} / \mathrm{UI}^{131}$.

The thyroids of these twenty controls with serum iodide levels around one microgram or less (without extra iodide) accumulated 5-16 micrograms $\mathrm{I}^{127}$ /hour, averaging 11 micrograms/hour. As serum iodide levels were raised to 6-12 micrograms per cent by iodide administration the iodine accumulated by the thyroid proportionally increased. With higher serum iodide levels inhibition of organic binding of iodide in the thyroid occurred, with subsequent complete blocking of uptake. This extended the observations of Wolff and Chaikoff in the rat. The thyroids in eight thyrotoxic patients accumulated an average of 122 micrograms $\mathrm{I}^{127}$ /hour; inhibitory serum iodide levels were less than five micrograms per cent.

With varying serum iodide values below inhibition the thyroid collects a constant fraction of the serum iodide. Probably the amount of iodide in the thyroid cells, rather than in the serum, determines the inhibitory point; with great ability to accumulate iodide (high thyroid: serum ratios) inhibition occurs with low serum iodide levels, and vice versa.

Fat in Intravenous Nutrition. Frederick J. STARE and (by invitation) Sherwood W. Gorens and Robert P. Geyer, Boston, Mass.

The inability to supply caloric requirements is a handicap in present-day parenteral therapy.

Work on the preparation of fat emulsions for intravenous use and on some of the metabolic aspects of the intravenous administration of fat has been under way in this laboratory. In the past year fat emulsions have been given to patients with varying degrees of success. A few cases will be mentioned of which the following is typical.

The patient was a 24-year-old white male with onset of frequent bloody mucous stools 7 years ago. A diagnosis of ulcerative colitis was made. Fifteen months prior to admission, he began to have 9 to 12 bowel movements per day and he lost 45 pounds. An ileostomy was performed. The ileostomy did not function properly, and the patient developed a peritoneal infection and a mid-jejunal fistula. It was necessary to reoperate to close the fistula. At this time the patient weighed only 90 pounds and was in an extremely poor state of nutrition. Oral intake was nil, and the usual parenteral therapy contributed no more than 400 calories per day. On the first postoperative day 300 $\mathrm{ml}$. of a 15 per cent fat emulsion containing 1.6 calories/cc. were given; on the second day twice this amount was given. The patient showed marked clinical improvement and felt much stronger. For the next 10 days he received daily infusions of fat varying from 600 to $900 \mathrm{ml}$. and furnishing 960 to 1,440 calories. By this time oral intake of food was sufficient and all parenteral therapy could be terminated.

Summarizing, fat emulsions can be prepared which can be given intravenously without reaction, and w!hich contribute appreciably to caloric intake without requiring large fluid volumes or prolonged infusion times.
The Serum Proteins in Portal Cirrhosis-Electrobhoretic Studies. Effects of Therapy. KenNeth Sterling and William E. Rickets (by invitation), and Joseph B. Kirsner and Walter L. Palmer, Chicago, Ill.

Electrophoretic analyses of the serum proteins and liver function tests were performed on ten normal subjects and twenty patients with proven portal corrhosis of the liver, with follow-up studies during the course of medical management in five patients.

The principal abnormalities observed in the serum proteins of 14 untreated patients were diminutions of the albumin fractions and elevations of the gamma-globulin fractions. Less frequently observed and usually less pronounced were elevations of the beta-globulin fractions. Of these 14 cases, seven with ascites and hyperbilirubinemia exhibited these alterations in marked degree, so that the gamma-globulin fractions usually approached or even exceeded the albumins. Seven patients without ascites manifested a considerable range of variation in the electrophoretic pattern from markedly abnormal to nearly normal in three cases without symptoms.

Serial electrophoretic studies and tests of hepatic function were done on five patients with ascites and hyperbilirubinemia during the course of medical management for nine months or less. Progressive change in the direction of normal coinciding with clinical improvement was observed during the administration of a high protein, high carbohydrate, high caloric diet. Infusions of plasma and concentrated human albumin were followed by pronounced and immediate elevations of the serum albumin values.

Six patients who had clinically recovered from severe portal cirrhosis and had been asymptomatic for more than two years were found to have electrophoretic patterns very close to normal.

These studies indicate that the alterations in the serum protein pattern in portal cirrhosis are not irreversible, but with prolonged medical management and clinical recovery, may revert practically to normal.

Alcohol and Homeostasis: The Uncompensated Water Diuresis Induced by Whiskey. MAURICE B. Str.iuss and (by invitation) Jack D. Rosenbaum and William P. Nelson, III, Framingham, Mass.

Although the diuretic effect of strong drink was commented upon by W. Shakespeare, modern scientific opinion has leaned toward the thesis that it is the large quantity of fluid so often simultaneously imbibed which provokes the increased urinary output. The occurrence of undue thirst following the ingestion of strong or even much diluted alcohol appears to be a matter of common knowledge albeit little concerning this may be found in scientific literature.

Five hours after breakfast, volunteers ingested $147 \mathrm{cc}$. of Bourbon whiskey or water. Following the former, the urine output was increased three-fold or more, but the excretion of strong electrolyte exhibited no significant deviation from the values observed after water.

In order to compensate for insensible water loss in the fasting, thirsting state, the urine should contain at least 
a third more electrolyte than the body fluids if hypertonicity of the latter is to be avoided. Since, after whiskey, the urine was observed to contain as little as one-fourth the electrolyte of body water and to be excreted in rather large volume, it is apparent that whiskey defeats the wisdom of the body and significantly disturbs homeostasis.

The rather constant glomerular filtration rate observed indicates that whiskey diuresis results from decreased tubular absorption of water. Evidence suggests that this is not due to a direct effect of whiskey on the tubules but rather an effect mediated through the supra-optico-hypophyseal mechanism.

No evidence was obtained of any alteration in the distribution of water between cells and extra-cellular fluid except that which would be anticipated as a result of the induced osmotic changes.

Both in the control and the whiskey studies the excretion of strong electrolyte often exhibited three-fold variation in spite of the fact that the amount of filtered electrolyte remained constant or varied in the opposite direction. Glomerular filtration of strong electrolyte would appear to be of less significance in controlling the rate of electrolyte excretion than has recently been suggested.

Studies of Formation, Diffusion and Absorption of Constituents of Cercbrospinal Fluid in Man. I. Studies with $\mathrm{Na}^{24}$. W. H. Sweet, B. Selverstone, A. SoloMON and L. BAKAY (Introduced by James C. White), Boston, Mass.

The rate of entrance of normal constituents into cerebrospinal fluid (CSF) from blood is being studied in man, with minimal disturbance of the hydrodynamic system. Needles placed in one lateral ventricle, cisterna magna and lumbar subarachnoid space remain in position throughout the experiment. Following intravenous injection of 16 microcuries of $\mathrm{Na}^{24}$ per kilogram, we withdraw at frequent intervals, samples of CSF not exceeding $0.2 \mathrm{cc}$. from each of the three points with concurrent blood samples. The final CSF pressure is not significantly altered from the initial level. Radioactive assay of specimens permits the drawing of satisfactory curves describing the rate of disappearance of $\mathrm{Na}^{24}$ from blood serum and its rate of appearance in CSF at each of the three points sampled.

Studies have been carried out not only on individuals in whom the circulation of CSF was presumably normal, but also in patients with "hypertensive meningeal hydrops," and in patients in whom a recent cerebral lobectomy for neoplasm had been carried out. Studies have been repeated after subsidence of the acute process in several patients.

A striking feature of the results has been the rapidity of appearance of $\mathrm{Na}^{24}$ in the lateral ventricle as contrasted with a slower rate in the cistern and an extremely slow rate in the lumbar subarachnoid space. In both the normal and the abnormal individuals the concentration of $\mathrm{Na}^{24}$ in the ventricle was three to four times that in the cistern at one hour, with an even greater differential prior to this time. In addition there appears to be an unexpectedly sluggish rate of flow and diffusion from the ventricles to the cisterna magna, since a number of hours elapse before the concentrations at the two points become the same.

A Proplasmin Activator from Mammalian Tissue. $\mathrm{H}$. J. Tagnon and (by invitation) Mary L. Petermann, New York, N. Y.

The proteolytic enzyme plasmin is present in normal blood plasma as an inactive precursor called proplasmin. Activation can be brought about by treatment of plasma with certain organic solvents or by the action of streptokinase. In certain pathological conditions, for instance traumatic shock, the active enzyme may appear in the circulating blood. This results in digestion of fibrinogen and fibrin and may lead to uncontrollable hemorrhage. The mechanism of activation of proplasmin in shock is unknown.

The experimental evidence presented here indicates that an activator of proplasmin is present in tissue, notably in lung. By differential centrifugation in the cold, it was possible to separate from an aqueous extract a particulate fraction possessing most of the activity of the extract while an inhibitor which was present in the extract remained in the supernate, and could thus be separated from the activator.

The activator was not removed from the particles by several washings. The activator transformed proplasmin into plasmin even in the presence of the plasmin inhibitor of plasma. Optimal pH range extended from 6.0 to 7.2. The temperature optimum was $37^{\circ} \mathrm{C}$. The amount of plasmin produced appeared to be proportional to the amount of activator and independent of the quantity of substrate (proplasmin). The activator lost its activity by incubation at $37^{\circ} \mathrm{C}$. for 4 hours or at $56^{\circ} \mathrm{C}$. for $1 / 2$ hour. It could be preserved indefinitely in the frozen state.

The presence of a proplasmin activator, probably a kinase, in tissue may explain the fibrinolysis in exudates and the resorption of blood clots from tissues. Whether such an activator will provide an explanation for the fibrinolysis of shock remains to be demonstrated.

\section{The Effect of Sodium Withdrawal upon the Body Weight of Normal Young Men. Caroline Bedell Thomas and (by invitation) Evelyn Howard and Arlene Is AAcs, Baltimore, Md.}

A disturbance of salt and water metabolism in hypertensive patients has been described by Perera and Blood. They reported that, following 24 hours of sodium restriction, the elimination of salt and water from the body as reflected in loss of body weight was much less marked in patients with hypertension than in normal subjects. Their work raises two questions :

1. In a larger group of normal subjects, do some respond to sodium withdrawal like hypertensive patients?

2. If such individuals be found, is there any evidence that they will eventually have hypertension?

The body weights of 83 male medical students were observed following 24 hours of sodium restriction (sodium chloride 1 gram) while on a standard diet. Among 64 un- 
selected students, a loss of 1.4 per cent of total body weight was the most frequent change observed. 47 lost 0.9 per cent or more, while 17 lost less than this amount, and in this respect resembled the hypertensive patients. Duplicate tests were carried out on 24 subjects, including 15 subjects showing the "hypertensive pattern" of response; results were consistent in 79 per cent of cases. A considerable proportion of normal young men, therefore, respond to sodium withdrawal with the "hypertensive" metabolic pattern.

A number of the unselected subjects had a history of hypertension affecting one parent, high normal resting blood pressure levels, a positive cold pressor test, or were overweight, and others selected because of these factors were included in the study. The correlation between these possible "prehypertensive" factors and the inability to lose weight following sodium withdrawal is discussed.

Correlation of Clinical Manifestations of Infection with the Elaboration of Hyaluronidase by Pathogenic Pneumococci. Robert T. Thompson and Frances E. Moses (Introduced by Morton Hamburger), Cincinnati, Ohio.

Pneumococci isolated from the eighteen to twenty-four hour blood cultures of thirty patients were tested for the ability to elaborate hyaluronidase by inoculation into 0.20 per cent hyaluronic acid broth. Hyaluronidase elaboration was determined by the mucoprotein clot prevention test. Twenty-one patients had primary pneumococcic pneumonia; nine others had pneumonia associated with some other disease. Cultures from ten primary pneumonia patients failed to elaborate hyaluronidase; one of these patients died. Cultures from eleven primary pneumonia patients elaborated hyaluronidase; three of these patients died. Considering all thirty cases, the duration of infection before blood culture seemed related to hyaluronidase elaboration by the infecting pneumococci. Of the cultures from five patients who had been ill only one day, one elaborated hyaluronidase and four did not. Of the cultures from eighteen patients who had been ill two to five days, nine elaborated hyaluronidase and nine did not. Of the cultures from seven patients who had been ill for seven days or longer, six elaborated hyaluronidase and one did not.

In the twenty-one cases of primary pneumococcic pneumonia, there was no relation between the age of the patients or the number of lobes involved and hyaluronidase elaboration by the infecting pneumococci. However, of the cultures from eleven patients with blood urea nitrogen under $40 \mathrm{mgm}$. per cent, four elaborated hyaluronidase and seven did not. Of the cultures of eight patients with blood urea nitrogen above $40 \mathrm{mgm}$. per cent, tive elaborated hyaluronidase and three did not. The blood urea nitrogen was not determined in two patients.

These findings indicate that pathogenic pneumococci are more likely to elaborate hyaluronidase in the infections of longer duration. In primary pneumococcic pneumonia hyaluronidase elaboration by infecting pneumococci is often associated with elevation of the patients' blood urea nitrogen.
The Influence of Streptomycin Dosage on the Pattern of of Emergence of Drug Resistant Tubercle Bacilli in Patients with Pulmonary Tuberculosis. RALPH TOMPSETT (Introduced by Walsh McDermott), New York, N. Y.

Demerec has shown that the development of streptomycin resistance of staphylococci in vitro proceeds by a series of stepwise increases in the degree of drug resistance of the bacterial population. In contrast with the homogeneity of steps in penicillin resistance, the first-step streptomycin resistant strains as well as those of subsequent steps contained some organisms of the highest degree of resistance. It is manifestly impossible to eliminate these highly resistant bacteria by the highest concentrations of streptomycin attainable in vivo. Nevertheless, it is conceivable that elimination of intermediate steps might effect a significant reduction in bacterial population during the time required for the highest step resistant strains to assume predominance, and hence prolong the effective period of drug action. It seemed of interest, therefore, to determine if a similar stepwise pattern of resistance is demonstrable in vivo, and the influence of streptomycin dosage on its appearance.

Observations have been made on strains of tubercle bacilli obtained from sixty-four patients with pulmonary tuberculosis treated with streptomycin. In 33 patients who received $3.0 \mathrm{gm}$. of streptomycin daily, the streptomycin resistance of all but one of the strains of tubercle bacilli fell into two well-defined steps. All strains were highly sensitive to streptomycin (2.0 micrograms per $\mathrm{ml}$.) prior to treatment. Strains obtained subsequently were found to remain at this level or to exhibit such a high degree of resistance that they were not inhibited by 100 micrograms per $\mathrm{ml}$. of streptomycin.

In contrast, a high proportion of strains obtained from 31 patients given only $1.0 \mathrm{gm}$. of streptomycin daily exhibited degrees of resistance intermediate between these two extremes. As many as half of the strains obtained during certain periods were found to be streptomycin resistant to various degrees less than the maximum.

\section{Effect of the Intravenous Injection of Vaccinia Virus on the Survival of Mice with a Transmissibie Leukemia. Joseph C. Turner and (by invitation) Barbara Mull- IKEN, New York, N. Y.}

In the course of investigations designed to examine the influence of extraneous viruses on experimental neoplasms, it appears that the behavior of lymphatic leukemia 9417 in inbred mice may be modified by the introduction into the hosts of certain strains of living vaccinia virus. In five separate experiments extending over a period of some months, of 148 untreated control animals 13 (9\%) survived for 30 days after the inoculation of leukemia and only $3(2 \%)$ for 60 days or more. In the same experiments 187 mice received single or multiple intravenous injections of suspensions of vaccinia at varying intervals after the transplantation of the leukemia. Of these, 49 $(26 \%)$ lived 30 days and $28(15 \%)$ survived 60 days or more. 
The variables involved are numerous and have not been fully resolved. The magnitude of the effect has so far appeared to be inversely proportional both to the acuteness of the leukemia and, perhaps paradoxically, to the virulence of the strains of virus. Thus, in the experiments noted above, vaccinia of low virulence, prepared from mouse-brain culture or frozen pulp (calf), were used. In other experiments highly virulent virus from rabbit-brain or egg-membrane have completely failed to influence the mortality of leukemic mice.

The mechanism of the observed effect remains uncertain; nor has it yet been settled whether the effect is determined by the infective particles themselves or some soluble component of the tissue suspension. Despite the difficulties attending the evaluation of findings in experiments with transmissible tumors, the point may be made that, as judged from an unequivocal end-point, survival, the behavior of a highly malignant neoplasm appears to have been modified in consequence of the introdurtion of a biological agent by the intravenous route at some time after the tumor may be assumed to have become established.

The Inheritance of Facioscapulohumeral Progressive Muscular Dystrophy; Variability in Expression in Human Inheritance as an Explanation of So-called "Anticipation." Frank H. Tyler (Introduced by G. E. Cartwright), Salt Lake City, Utah.

A study has been made of a large kindred, certain members of which have facioscapulohumeral dystrophy. There are 1,249 individuals in six generations in the established pedigree. The inheritance is typical of a Mendelian dominant trait with complete penetrance and variable expressivity. Among the completely or nearly completely studied sibships which represent simple back crosses ( $\mathrm{Dd} \times \mathrm{dd}$ ) there are 130 dystrophies and 143 normal individuals. The deviation from the expected $1: 1$ ratio is insignificant.

The disorder in the average patient is only slightly incapacitating. However, there is great variabilicy in the severity of its expression in middle and old age. Even the patients with the more severe disabilites of the disease have had large families. This fact has permitted an enlightening comparison of the severity of the disease in the offspring and in the parent. This has shown that in certain families the children were more severely involved than the parents. In other families, the parents show the most marked changes.

In families in which diabetes mellitus or myotonia dystrophica occur, it has long been assumed that the disorder becomes progressively more severe in successive generations. This phenomenon has been called anticipation or antedating. Another explanation for the observed variation in these diseases is variability in the expression of the trait and the fact that in severe cases, beginning in adolescence, procreation becomes highly improbable. Comparison with the findings in facioscapulohumeral dystrophy where large families can be studied, suggests that this is the true explanation rather than "anticipation."
The Use of Emission Spectrography in the Study of Metabolism of Metals in the Blood in Man. BERT L. VALlEE and JoHN R. Loofbourow (Introduced by M. D. Altschule), Boston, Mass.

Emission Spectrography is based on the principle that high energy excitation of atoms causes them to emit light of characteristic wavelengths; each element has a typical spectral pattern which may be recorded photographically. This technique permits the simultaneous identification of practically all elements present in a given sample. Quantities as low as fractions of one microgram may be detected accurately. Quantitation of at least five or six elements simultaneously is possible.

Previous use of this technique resulted in identification of Zinc in leucocytes. Now in progress are studies of the distribution of elements in the fluid and corpuscular components of human blood. Studies have been made on Magnesium, Manganese, Copper, Strontium, Rubidium, Titanium and other metals regularly found in blood. Some of these elements, heretofore, have not been thought to be of biological significance.

The results of the present investigation are of interest in view of the known relation of metals to various enzyme systems present in erythrocytes and leucocytes. In some instances, the element is an integral part of the enzyme molecule such as in the case of iron in catalase, cytochromes, verdoperoxidase, zinc in carbonic anhydrase and copper in tyrosinase. In others, in vitro activation by various metallic ions has been found essential as exemplified by arginase, phosphatase and succinic dehydrogenase.

The significance of our findings in regard to the metal enzyme relationships will be discussed.

\section{The Iron Metabolism and Acid Fragility of Erythrocytes in Paroxysmal Nocturnal Hemoglobinuria. PHILIP F. Wagley and Margaret Adams (by invitation), and Joseph F. Ross, Boston, Mass.}

Two subjects (L. G. and L. T.) with paroxysmal nocturnal hemoglobinuria were given radioactive iron by parenteral injection and samples of their blood were taken at subsequent intervals. For a time the maximum possible age of the erythrocytes containing radioactive iron would correspond to the interval between the injection and the sampling. Washed erythrocytes derived from aliquots of blood taken at specified intervals after the injection of radioactive iron were subjected to hydrochloric acid hemolysis at a $\mathrm{pH}$ of $6.7-6.9$ and determinations were made of the relative amounts of hemoglobin and of radioactive iron released. During a short period following injection, acid hemolysis released two to three times more radioactive iron from the red cell mass than could be accounted for by release of hemoglobin.

Most of this radioactive iron released by acid hemolysis was not loosely bound ("easily splittable iron") ; 73-96 per cent of the radioactivity was precipitated with the proteins by trichloracetic acid.

These observations have been considered in light of two possibilities: (1) the susceptibility of the red blood 
cell to acid hemolysis may vary with age of the cell, or (2) all of the radioactive iron released by acid hemolysis is not in hemoglobin, some being in other protein bound form(s). The implications of these possibilities will be discussed.

The Effect of Auricular Fibrillation on the Coronary Circulation. RENé WÉgria and (by invitation) RICHARD P. Keating, Henry P. Ward and Charles W. Frank, New York, N. Y.

Forty-five bouts of auricular fibrillation induced in twelve dogs were studied as to their effect on the mean arterial blood pressure and coronary flow.

At the onset of auricular fibrillation, a marked drop in blood pressure and coronary flow occurs. Then, within a few seconds, both blood pressure and coronary flow return toward their control levels, but whereas the coronary flow may, at least temporarily, return to or even rise above its control level, the blood pressure very seldom reaches it. As soon as fibrillation stops, both coronary flow and blood pressure rise above control levels, then come down progressively to their control level, but the blood pressure may come back to its control level before the coronary flow. The mechanisms and significance of the cardiovascular reactions observed will be discussed.

Growth Forms of Common Bacteria Indistinguishable from Pleuropneumonia-Like Organisms. How ARD J. Weinberger and Louis Dienes (Introduced by J. H. Means), Boston, Mass.

The authors will present additional evidence indicating that many commonly occurring bacteria can survive and multiply in a peculiar form not recognizable by the usual bacteriological methods.

Freshly isolated strains of many bacillary species transferred to appropriate media containing varying concentrations of penicillin undergo a peculiar transformation which results in the development of colonies indistinguishable from those of the Pleuropneumonia group. Strains of Typhoid, Salmonella, Shigella, H. influenzae, Proteus, Bacteroides, Flavobacterium, Streptobacillus moniliformis, and a Gram positive spore bearing bacillus exhibited this phenomenon. In Salmonella, Proteus, Bacteroides and Streptobacillus these Pleuropneumonia-like (L) forms can be made to revert to the usual bacillary forms indicating that they are growth forms of the bacteria despite the marked differences in their morphology, staining reactions, physical properties, and growth and reproductive characteristics.

The effect of penicillin is not specific. Similar transformation occurs under other "untoward" influences including autolysis of the cultures. Exposure of typhoid bacilli to fresh human serum also has resulted in the development of typical $\mathrm{L}$ colonies.

The Pleuropneumonia group is responsible for important animal diseases. In humans Pleuropneumonia-like organisms have been recovered from prostatic, urethral and vaginal secretions, bladder and kidney urine, joint fluid, empyema fluid, paronychial and peritoneal abscesses and sputum.
The nature of these organisms is not fully understood and in most cases the relation of the organisms isolated from pathological processes to a parent form has not been established. The pathological significance of bacteria in the Pleuropneumonia-like form warrants intensive study. Such organisms have been observed under conditions similar to those prevailing in the body. In light of the widespread therapeutic use of penicillin and the frequent incorporation of penicillin in culture media, it is possible that the incidence of Pleuropneumonia-like organisms will increase.

Studies of the Influence of Penicillin on the Immune Reactions in Streptococcal Pharyngitis. LouIS WeINSTEIN and (by invitation) LouIs BACHRACH and Thomas S. Perrin, Boston, Mass.

A group of 225 patients with pharyngitis due to the beta-hemolytic streptococcus was treated with penicillin given in a dose of 15,000 units intramuscularly every 3 hours for 10 days. Serial examinations of their serums for antistreptolysin, antistreptokinase, anti-M, and anti-C substance antibody and the "phase" reacting material of Coburn, at weekly intervals for a month or more revealed that there was a marked reduction in the ability to produce all of these antibodies. In contrast to the 75-85 per cent incidence of antistreptolysin and the 2535 per cent incidence of antistreptokinase in control untreated patients, of those given penicillin only about 12 per cent developed significant antistreptolysin and/or antistreptokinase titers. In the treated individuals who showed an increase in either or both of these antibodies, the height of the titer attained was considerably lower than in the average control patient. None of the treated group developed any anti-M or anti-C substance antibody and the "phase" reactions indicated the lack of value of this test as a diagnostic aid in rheumatic fever. About 7 per cent of the treated individuals developed rheumatic fever while approximately 6 per cent showed evidence of a varying degree of glomerular nephritis; of these patients, only about $1 / 3$ had a rise in antistreptolysin or antistreptokinase in the blood.

When the penicillin treatment schedule was changed to administration every 8 hours either intramuscularly or orally, the number of individuals showing significant antistreptolysin titers increased remarkably. Thirty-two per cent of 103 patients given 100,000 units intramuscularly every 8 hours showed an increase in this antibody. Exhibition of penicillin by mouth on an 8-hour schedule showed an even lesser tendency to inhibit antibody formation and in a group of 241 patients given the drug in doses varying from 50,000 to 800,000 units, from 40 to 80 per cent showed an increase in antistreptolysin. These differences in antibody formation could not be correlated with persistence or recurrence of streptococcal infection since the vast majority of the patients were rid of their organisms in $24-48$ hours after therapy was instituted. That the increased incidence of antibody formation may be due to longer persistence of Streptococci in the pharynx is suggested, however, in the group receiving 50,000 units of penicillin orally every 8 hours since, in these pa- 
tients, recurrence of the organisms in the pharynx was common after treatment was stopped. A small number of individuals receiving oral penicillin showed a rise in the anti-M substance antibody.

The Fate of Transfused Leukocytes. Austin S. WeisBERger (by invitation), ROBERT W. Heinle and (by invitation) JOHN P. StoraAsLI and Richard HANNAH, Cleveland, Ohio.

Elevation of the leukocyte count following whole blood transfusions does not occur even in severely leukopenic recipients. A study of this phenomenon was undertaken. Concentrated suspensions of leukocytes were obtained from the peritoneal cavity of rabbits by distending their abdomens with physiologic saline. If radioactive phosphorus $\left(\mathrm{P}^{32}\right)$ was administered previously in doses of 1 millicurie, the cells obtained were radioactive. The fate of such transfused cells was studied by determining the radioactivity of tissues of recipient rabbits.

The peritoneal fluid was collected, centrifuged and washed. Practically all of the $\mathrm{P}^{32}$ was fixed in the cells. These were resuspended in Tyrodes solution and transfused into the same or other rabbits.

Following the intravenous injection of radioactive or non-radioactive leukocytes, an initial increase of blood leukocytes never occurred. Instead, a profound leukopenia developed usually within 1 to 5 minutes. In more than half the animals a subsequent leukocytosis occurred after 4 to 6 hours. In 5 rabbits sacrificed during the leukopenic stage, the distribution of radioactivity was: lung, 45.4 per cent ; liver, 20.7 per cent ; spleen, 1.0 per cent. In marked contrast, the distribution following injection of inorganic $\mathrm{P}^{32}$ was : lung, 1.3 per cent; liver, 11.9 per cent; spleen, 0.33 per cent. Following intra-aortic injection of radioactive leukocytes into 5 rabbits, the distribution of radioactivity was: lung, 30.5 per cent; liver, 17.8 per cent; spleen, 0.35 per cent. Following disappearance of the leukopenia ( 4 to 6 hours), the distribution in 5 rabbits was : lungs, 19.1 per cent ; liver, 34.9 per cent ; spleen, 3.4 per cent.

These findings suggest that the lung plays an active role in controlling the blood leukocyte level. Since cell-free aqueous extracts of leukocytes produce a similar profound leukopenia, the arrest of leukocytes in the lungs represents more than a simple filtering of foreign bodies.

Effects of Infusion of Albumin on the Excretion of Water and Electrolytes in Normal Subjects. Louis G. WeLt and JACK OrlofF (Introduced by John P. Peters), New Haven, Conn.

During infusions into normal subjects of 100 grams of a 25 per cent solution of salt-poor albumin the rate of excretion of sodium decreases. There is no significant alteration in water excretion. These changes are associated with an increase in plasma volume. They are not correlated with the degree of change in total protein or albumin concentrations in the serum nor with a significant change in endogenous creatinine clearance.

Administration of a 10 per cent solution of albumin resulted in an increase in plasma volume and a definite de- crease in the rate of excretion of sodium and water. In contrast, when albumin was administered as a 4 per cent solution water excretion rose strikingly and sodium excretion questionably. This reaction was less marked when a 5 per cent solution was administered.

It appears that when albumin is infused in normal subjects in concentrations with a higher colloid osmotic pressure than that of serum the rate of water and sodium excretion diminishes below that obtained with iso-osmotic albumin infusions. When the plasma volume is expanded, but the colloid osmotic pressure remains normal the kidneys respond in such a manner as to correct over-hydration; but when expansion of plasma volume is accompanied by excessive colloid osmotic pressure their response is adjusted to correct dehydration. Gradations of these reactions elicited by varying the concentrations of injected albumin suggest that the responses are controlled by the balanced action of two forces, colloid osmotic pressure and plasma volume.

It is also possible that a decrease in interstitial fluid volume in normals initiates a mechanism that increases the reabsorption of sodium and water; and an increase in plasma volume unassociated with a decrease in interstitial fluid volume provokes a diuresis.

A Comparison in the Same Individual of the Nitrogen Loss Following Surgery with that Produced before Operation by an Identical Feeding Program. Sidney C. Werner, New York, N. Y.

Twenty-eight patients on approximately adequate diets in terms of calories and protein have been given a reduced diet similar to that usually given post-operatively, both before and after the procedure. The resultant alterations in nitrogen balance have been compared. No significant increase in negative nitrogen balance occurred after cholecystectomy, inguinal or extensive ventral hernia repair, provided constant feeding was maintained through the operative day and subsequently.

The data obtained suggest that most of the work during the war concerning the effect of trauma or disease on nitrogen balance cannot be interpreted as indicating the role of injury. First, the preceding dietary intake was often not known. Second, a constant intake of calories and protein was not maintained from the moment of trauma. Third, the increased demands for calories resulting from fever have not been taken into account, in most studies.

Since no changes in nitrogen balance after operation have been found which could not be accounted for on the basis of inadequate calories, a review of the typhoid fever work on "toxic destruction of proteins" has been made. Here too, adequate provision of calories produced nitrogen equilibrium at the height of disease, a finding similar to the war period findings in this and other laboratories on the effect of increased protein, or calorie and protein, intake after injury or operation. The one study suggesting that an excess of calories is needed in typhoid fever to restore nitrogen equilibrium is vitiated by the fact that the patients lost weight on a theoretically adequate diet.

It is thus suggested that the "catabolic response" or "toxic destruction of proteins" is probably the result of 
lack of provision of adequate calories, protein, or both, and not of unusual body mechanisms being brought into play; or if such effects are present, that, divorced from under-nutrition, they are not highly significant.

Antidiuresis and Renal Vasoconstriction Following Venous Congestion of the Limbs in Normal, Hypertensive, and Splanchnicectomized Subjects. RoBERT W. WILKins, (by invitation) James W. Culbertson, Belton A. Burrows, Clarence M. Tinsley and Walter E. Judson, and Charles H. Burnett, Boston, Mass.

When the legs of supine subjects were congested by blood pressure cuffs inflated on the thighs at less than diastolic pressure for 10 to 20 minutes, there was usually little change in arterial pressure or pulse rate, and little effect on renal circulation or function until after the cuffs were released. Ten to twenty minutes after the release of the congesting cuffs there was a marked antidiuresis (as much as 90 per cent), a decrease ( 25 to 60 per cent) in renal plasma flow (PAH) and filtration rate (inulin, mannitol), and a reduction of sodium, potassium and chloride excretion (as much as 60 per cent). In 10 to 20 minutes the changes in renal circulation and electrolyte excretion had usually disappeared, but the antidiuresis persisted for at least 40 minutes.

Similar renal functional and circulatory effects followed venous congestion of the limbs of hypertensive subjects before and after lumbodorsal sympathectomy.

These results appear significant in connection with the various mechanisms of water and salt retention and the disturbances of renal circulation in venous congestive myocardial failure. They indicate that venous congestion of the limbs, and possibly of other peripheral areas, may contribute to the causes of reduced renal plasma flow and excretion of water and salt in this condition. Apparently these renal disturbances may persist for some time after the relief of venous congestion. They are not necessarily preceded by changes in renal arterial or venous pressure, and are not mediated over the splanchnic sympathetic nervous system since they are present after splanchnicectomy. Possibly they are mediated through humoral mechanisms.

The Application of a Strain Gange Manometer, a Modified Ear Oximeter and Whole Blood Cuvette Oximeter to the Technic of Cardiac Catheterization. EARL H. Wood, Edward Lambert and Howard B. Burchell (Introduced by Charles F. Code), Rochester, Minn.

A strain gauge manometer (Statham Model P6-25015D) coupled without amplification to sensitive galvanometers affords one of the most accurate and simple means of recording pressure through a cardiac catheter. Uniform sensitivity to sine wave variations in pressure of from 0 to 28 cycles per second may be obtained through a cardiac catheter if proper electrical and mechanical damping are used. This is superior to the dynamic response obtained with a Hamilton manometer ( natural frequency: 120 cycles per second). The strain gauge manometer is connected to (1) a visual indicating galvanometer utiliz- ing a red light thus permitting continuous monitoring of pressures during roentgenoscopy and (2) an oscillographic galvanometer located in an adjacent recording room which permits simultaneous and continuous photographic recordings of the variations in pressure transmitted through the catheter. It is recognized, however, that the physical characteristics of the catheter and the pressures generated within its lumen by the acceleration and deceleration of the fluid column associated with the movements of the catheter imparted by the heart beat obviate the possibility of obtaining uniformly reliable tracings of intracardiac pressure with manometers attached to the external end of the catheter.

Variations in arterial saturation are recorded continuously on the oscillograph and monitored simultaneously from a visual reading scale by means of a modified ear oximeter.

The oxygen saturations of samples of blood withdrawn from the catheter are recorded and monitored similarly with a whole blood cuvette oximeter. The electrocardiogram and respiration are recorded simultaneously.

Immediate correlation of oxygen saturation of samples of blood withdrawn from the catheter with associated observations of pressure, arterial saturation, and location of the tip of the catheter (roentgenoscopy) greatly expedites the procedure. The probable diagnosis was apparent prior to withdrawal of the catheter in the majority of cases in which analysis of all data ultimately proved sufficient for such a decision to be made.

The Chemoprophylaxis of Scrub Typhus in Volunteers. T. E. Woodward and H. L. Ley JR. (by invitation), and J. E. Smadel, Washington, D. C.

Two field trials were conducted in Malaya in which 75 volunteers were exposed in hyperendemic areas of scrub typhus. Thirty-four of these individuals received Chloromycetin prophylactically for a total period of three weeks during and following exposure. They did not show clinical evidence of scrub typhus throughout the period of prophylaxis or the ensuing week. However, scrub typhus developed in 15 volunteers in the prophylactic groups of the two trials 5 to 14 days after drug was discontinued. Although the attack rate among the volunteers in the two field trials varied markedly, there was no essential difference in the ultimate infection rates among the controls and those given prophylaxis in each test.

Scrub typhus, when it developed among volunteers in the prophylactic group, was not significantly different from the disease in the controls except for the absence of eschar formation.

Relapses were prominent features of the disease in the volunteers of both prophylactic and control groups. These had not been observed previously in untreated cases of scrub typhus or in naturally occurring cases which were treated with chloromycetin. Fifty-four per cent of the 37 persons in the two trials who contracted scrub typhus suffered one or more relapses. Various factors probably contributed to this phenomenon.

Prolonged administration of chloromycetin as a prophylactic measure and its use in the treatment of the initial 
attacks of scrub typhus as well as the relapses indicated that the drug is of low toxicity for man, and that drugfast strains of $R$. tsutsugamushi are not readily produced.

Experimental Production of Hemolytic Disease in Newborn Dogs. Lawrence E. Young and (by invitation) Donald M. Ervin, Richard M. Christian and $R$. Wendell Davis, Rochester, N. Y.

Isohemagglutinins of 4 different specificities have been demonstrated in sera of dogs following transfusions of dog erythrocytes containing antigenic factors lacking in their own cells. Since the behavior of one of these antibodies is in some respects similar to that of $\mathrm{Rh}$ antibodies, dog antisera having this specificity have been tentatively designated "anti-Do," and dog cells have been classified as "Do-positive" or "Do-negative" depending upon their reaction with such sera.

Hemolytic disease has been produced in Do-positive puppies born to a Do-negative dam immunized by transfusions of Do-positive cells before mating with a Dopositive sire.

In the first litter investigated, erythrocytes of 4 affected Do-positive whelps were agglutinated by high dilutions of anti-dog-serum rabbit serum and were also agglutinated when suspended in undiluted normal dog serum, presumably because the cells were coated with Do-antibody. Plasma from 4 unaffected Do-negative whelps in the same litter agglutinated the sire's cells, but iso-antibodies were not detectable in the plasma of the affected Do-positive puppies. The dam's breast milk agglutinated the sire's erythrocytes but absorption of iso-antibody from milk by the puppies could not be demonstrated.

The degree of anemia and icterus varied markedly among the affected whelps of the same litter. Erythrocytes of all of the Do-positive puppies, however, showed increased osmotic fragility, and reticulocytes and nucleated red cells were more numerous in venous blood of the affected puppies. Autopsy of a severely anemic whelp and of a normal litter mate revealed more marked erythropoiesis in liver, spleen and marrow of the affected animal.

Observations thus far completed suggest that studies on hemolytic disease in newborn dogs may aid in clarifying certain aspects of the comparable human disorder, and that controlled investigations on large litters sired by heterozygous males may be especially productive.
Intrathoracic Pressure Change as an Aid in the Anatomic Localization of Murmurs and Other Sounds of Cardiovascular Origin. HARRY F. ZinsSER, JR. (by invitation) and Calvin F. Kay, Philadelphia, $\mathrm{Pa}$.

Certain commonly used diagnostic techniques are based on the relationship of murmur intensity to rapidity and volume of blood flow. By exercise, amyl nitrite and other means, circulatory velocity is increased to accentuate murmurs. If blood flow could be altered differentially in the lesser and greater circulations, the time of murmur accentuation should provide a clue to its anatomic origin. Such changes in circulatory dynamics occur in the relaxation period immediately following voluntary straining.

During straining, venous blood return is decreased. The right heart output falls, the pulmonary reservoir is depleted, and systemic output falls. When intrapulmonic pressure returns to atmospheric level, venous blood rushes first into the right heart, causing vigorous pulmonary artery pulsations and restoring the lesser circulation. Left heart output and aortic pulsations become maximal only after the pulmonary blood reservoir is restored.

It was predicted, therefore, that murmurs should become faint during straining and gain intensity after relaxation. Murmurs of right heart or pulmonic origin should become intense immediately in the post-straining period, while return of left sided or peripheral murmurs should be delayed.

Studies were undertaken with combined phonocardiographic, electrokymographic, and electrocardiographic techniques upon subjects with murmurs of either known or unknown origin. Variations in intrathoracic pressure produced by straining against a mercury manometer were recorded with strain-gauge attachment.

Recordings bear out the predicted results. Murmurs were loudest either early, associated with maximal electrokymographic pulmonary artery pulsations; or late, coinciding with maximal aortic pulsations. Certain patients fail to produce effective straining. Where straining was effective, the method successfully differentiates known right sided from left sided murmurs. In patients with murmurs of unproven type, equally decisive results seem significant by deduction. Alterations of other cardiac sounds also occur, similarly suggesting the site of production. 